\title{
On the Stability of Julia Sets of Functions having Baker Domains
}

\author{
Dissertation \\ zur Erlangung des Doktorgrades \\ der Mathematische-Naturwissenschaftlichen Fakultäten \\ der Georg-August-Universität Göttingen
}

vorgelegt von

Arnd Lauber

aus Bremen

Göttingen, 2004 
D7

Referent: PD Dr. Hartje Kriete

Koreferent: Prof. Dr. Manfred Denker

Tag der mündlichen Prüfung: 
This thesis deals with Baker domains and approximation of Julia sets, so it belongs to the area of "Holomorphic Dynamical Systems". Dynamical systems belong to the all-day life of scientists and engineers and are closely related to the term of iteration.

Processes of iteration occur if the state of a system is changed by external influences at discrete points of time. Examples are the weather, turbulent flows in liquids or the development of populations.

Moreover, iteration can be a tool to solve other mathematical problems or to approximate their solutions. Among various numerical methods we only mention Newton's method to approximate roots of differentiable functions. All these dynamical systems have in common that they may develop in different directions. The boundary between different initial states of different developments is just the Julia set of the corresponding function.

Julia sets were systematically analyzed for the first time around 1920 by the French matematicians P. Fatou and G. Julia, who concentrated on rational functions and observed that Julia sets are either very simple objects or extremly complicated. The development of powerful computers and new mathematical methods gave a boost to the research in this area in the 80's, and since then also a theory of iteration of entire transcendental functions has been founded.

The aim of this work is to describe what can happen to the Julia sets if an entire transcendental function satisfying a certain condition (having so-called Baker domains or wandering domains) is approximated by a sequence of polynomials or is perturbed holomorphically in a class of entire transcendental functions. 


\section{Organization of the paper}

This paper comes in four chapters: the Introduction, Preliminaries and Notation, the Results and the Proofs.

All the results will be stated in chapter 3, and, for example, a result in section 3.1.1 will have its proof located in section 4.1.1.

\section{Acknowledgements}

I would like to thank my supervisor Hartje Kriete for giving me this interesting topic and his encouragement. Also a lot of thanks to the group in Barcelona I was working with, especially to Nuria Fagella, Guillermo Sienra, Patricia Dominguez and Antonio Garijo for many very fruitful discussions. I was staying at universities in Barcelona, Paris, Mexico and Rio de Janeiro, thanks to everyone who made this possible. Thanks to everyone I had the pleasure to work with, and especially to Jordi and Toni for valuable help on the programming issues.

Finally, thanks to Mum and Dad.

This reseach was funded by a Marie-Curie-Grant and grants from the DAAD. 


\section{Contents}

\begin{tabular}{lll}
\hline & Introduction & 7
\end{tabular}

2 Preliminaries and Notation $\quad 11$

2.1 Background in Complex Dynamics . . . . . . . . . . . . . 11

2.2 Baker domains and wandering domains . . . . . . . . . . . 16

2.3 Notation . . . . . . . . . . . . . . . . . . . . . . . 22

3 Results $\quad 23$

3.1 A one-parameter family . . . . . . . . . . . . . . . . 23

3.1.1 The dynamical plane . . . . . . . . . . . . . . . 23

3.1 .2 The parameter plane . . . . . . . . . . . . . . . . . . 28

3.1 .3 A related family . . . . . . . . . . . . . . . 30

3.2 Generalization . . . . . . . . . . . . . . . . . . . . . 34

3.3 Approximation of Julia sets . . . . . . . . . . . . 37

3.3.1 Preliminaries . . . . . . . . . . . . . . . 37

3.3 .2 Known results . . . . . . . . . . . . . . . . . . 39

3.3.3 Approximation of functions having Baker domains . . . 41

3.4 Bifurcations of Baker domains . . . . . . . . . . . . . 47

3.4 .1 The dynamical plane . . . . . . . . . . . . . . . . 47

3.4.2 Radial continuity of Julia sets . . . . . . . . . . . 49

3.4.3 Approximation of Baker domains and wandering domains 52

3.4.4 Stability . . . . . . . . . . . . . . 55

3.5 Open questions . . . . . . . . . . . . . . . . 57

$\begin{array}{lll}4 & \text { Proofs } & 59\end{array}$

4.1 A one-parameter family . . . . . . . . . . . . . . . . . . . . 59

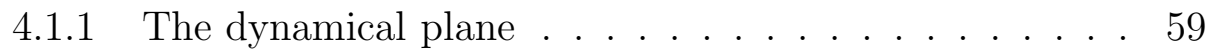

4.1.2 The parameter plane . . . . . . . . . . . . . . 80

4.1.3 A related family . . . . . . . . . . . . . . . . 88

4.2 Generalization . . . . . . . . . . . . . . . . . . . . . . 90

4.3 Approximation of Julia sets . . . . . . . . . . . . . . 93 
4.3.3 Approximation of functions having Baker domains . . . 93

4.4 The limiting processes $\operatorname{Re} a c \rightarrow 0$. . . . . . . . . . . . . . 104 4.4 .1 The dynamical plane . . . . . . . . . . . . . . . . 104

4.4 .2 Radial continuity of Julia sets . . . . . . . . . . 107 4.4.3 Approximation of Baker domains and wandering domains 112 4.4 .4 Stability . . . . . . . . . . . . . . . . 113 


\section{Chapter 1}

\section{Introduction}

For any polynomial $f: \overline{\mathbb{C}} \rightarrow \overline{\mathbb{C}}$ or any entire transcendental function $f: \mathbb{C} \rightarrow \mathbb{C}$ we can divide the plane into the Fatou set, roughly speaking the set of points which are locally stable under iteration, and the Julia set. In the case of polynomials, the structure and type of components of the Fatou set are quite well understood, for entire transcendental functions there are additional possibilities of types of Fatou components, namely Baker domains and wandering domains, where questions remain open.

A natural question to ask is how the nature of a dynamical system obtained by iteration changes if the studied function is perturbed. A lot of efforts has been put into studying this question for holomorphically parameterized functions of polynomials, culminating in the study of the famous Mandelbrot set. Although there are some important open problems left in this area, also the interest in families of entire transcendental functions has risen. A lot of analogies to the polynomial case have been found, including the existence of so-called copies of the Mandelbrot set for families of entire transcendental functions.

A question related to this area can be formulated as follows: If a sequence of entire functions $f_{n}$ converges to some function $f$, do we have $J\left(f_{n}\right) \rightarrow J(f)$ ? Some partial answers have been given, the answer is affirmative if the Fatou set of the limit function is empty or consists of attracting basins only. The problem is more delicate if the Fatou set of the limit function contains Leau domains or Siegel disks, leading to the well-studied phenomenon of the "parabolic implosion". The Julia sets do not necessarily converge in this case, depending on the sequence $f_{n}$.

For Baker domains and wandering domains a deeper study of possible scenarios is still missing, examples both with possible answers have been given by Morosawa.

In the first section we will study the family of entire transcendental functions 
given by $\mathcal{F}_{b}=\left\{g_{b}: \mathbb{C} \rightarrow \mathbb{C}, g_{b}(z)=z-1+b z e^{z}\right\}$. All of these functions feature Baker domains. Firstly we will examine the corresponding dynamical planes. Then we will see that it is possible to reasonably define sets in the parameter plane (in analogy to the quadratic polynomials), and deal with global questions on the parameter plane, in particular, we will establish the existence of a copy of the Mandelbrot set. For a closely related family this will imply the existence of a sequence of Mandelbrot copies tending to a finite point. Furthermore, at this point our results will be sufficient to find a partial answer on the (local) question of convergence of the Julia sets in this family. To the best of our knowledge, this is the first example of this kind on families of functions having Baker domains.

In the second section we prepare ourselves to studying this question of convergence of Julia sets for a wider class of limit functions, namely the class $\mathcal{F}=\left\{f: \mathbb{C} \rightarrow \mathbb{C}: f(z)=z-c+P(z) e^{a z}, \operatorname{Re} a c>0,0 \not \equiv P\right.$ a polynomial $\}$, by looking at the dynamical planes of these functions, in particular, at Baker domains in each of the corresponding Fatou sets.

We continue with studying sequences of polynomials tending to these entire transcendental functions and consider the sequences of Julia sets of the polynomials. Of course, if these Julia sets converge to the Julia set of the limit function, then one can ease the examination of the dynamical system induced by the transcendental limit function by studying the Julia sets of the polynomials insted, which is considerably simpler.

Our main result will be that it depends on the choice of the polynomials if the Julia sets converge. More precisely, for each function $f$ in $\mathcal{F}$ we will give two sequences of polynomilas, for one sequences the Julia sets do not converge to the Julia set of $f$, while for the other one at least the Baker domains are approached (in a way we will define later). We will give sufficient conditions for the convergence of the whole Julia sets in this case.

Beside the convergence of polynomials to entire transcendental functions, it is also interesting to pick up the case of entire transcendental functions tending to an entire transcendental function. In the first section we consider a family of functions all having Baker domains, in the fourth section we study the family $F_{c}=\left\{f_{c}: \mathbb{C} \rightarrow \mathbb{C}, f_{c}(z)=z-c+e^{z}\right\}$. We already know that some of these functions do have Baker domains, but some do not, so the question arises what happens in the points of bifurcation. In this family there are four different scenarios of bifurcations, interestingly the Julia sets change their topological nature drastically (concerning the number of Fatou components as well as their type), but the Julia sets move continuously in each case. The methods we will use provide us with a direct link to the bifurcation of Baker domains and the bifurcation of Leau domains, the parabolic implosion. 
We then extend our results of the previous section to a subset of our new family, this time we even obtain results on the convergence of Julia sets of polynomials to the Julia sets of limit functions having wandering domains. Finally, we use our results to think about a question raised by König: He classified Baker domains in three categories and asked if there is a category containing only Baker domains that are stable under perturbation; we will give examples to show that this is not the case, a more detailed study of this problem gives some more concrete results. 


\section{Chapter 2}

\section{Preliminaries and Notation}

\subsection{Background in Complex Dynamics}

Let $f: \mathbb{C} \rightarrow \mathbb{C}$ (or $f: \overline{\mathbb{C}} \rightarrow \overline{\mathbb{C}}$, respectively, in case of a polynomial) be an entire function, but not a constant or linear map. We denote the $n$-th iterate of $f$ with $f^{n}$, that is, $f^{0}(z)=z$ and $f^{n+1}(z)=f\left(f^{n}(z)\right)$ for $n \geq 0$. This induces a partition of the complex plane into the Fatou set $F(f)=\left\{z:\left\{f^{n}\right\}_{n \in \mathbb{N}}\right.$ forms a normal family in a neighborhood of $\left.z\right\}$ and its complement, the Julia set $J(f)=\overline{\mathbb{C}} \backslash F(f)$. A special role is played by the periodic points of $f$. A point $z \in \mathbb{C}$ is called periodic of period $p$ if $f^{p}(z)=z$ and $p$ is the smallest integer having this property. A periodic point $z$ of period $p$ is called indifferent if $\left|\left(f^{p}\right)^{\prime}\left(z_{0}\right)\right|=1$. If $\left(f^{p}\right)^{\prime}\left(z_{0}\right)=e^{2 \pi i \alpha}$ for some $\alpha \in \mathbb{Q}$, it is a parabolic (or rationally indifferent) periodic point, if $\left(f^{p}\right)^{\prime}\left(z_{0}\right)=e^{2 \pi i \alpha}$ for some $\alpha \in \mathbb{R} \backslash \mathbb{Q}, z_{0}$ is called irrationally indifferent and is either the center of a Siegel disk (see below) or it is contained in the Julia set. In the latter case it is called a Cremer point. If $f^{p}(z)=z$ and $\left|\left(f^{p}\right)^{\prime}(z)\right|<1, z$ is an attracting periodic point, in the special case $\left(f^{p}\right)^{\prime}(z)=0 z$ is called a super-attracting periodic point.

We give some basic features of the Fatou and the Julia set:

- $J(f)$ and $F(f)$ are completely invariant, that is, $z \in J(f) \Leftrightarrow f(z) \in J(f)$ and $z \in F(f) \Leftrightarrow f(z) \in F(f)$

- $F(f)$ is open and $J(f)$ is closed

- $J(f)=\partial F(f)$ or $J(f)=\overline{\mathbb{C}}$

- the Julia set is equal to the closure of the set of repelling periodic points

- $F(f)=F\left(f^{n}\right)$ and $J(f)=J\left(f^{n}\right)$ for $n \geq 1$ 
- $J(f)$ is the smallest closed, completely invariant set containing at least three points. In fact, $J(f)$ is always uncountable

- for any compact set $K \subset \mathbb{C}$ and open set $U \subset \mathbb{C}$ with $U \cap J(f) \neq \emptyset$ we have $f^{n}(U) \supset K$ for all but finitely many $n \in \mathbb{N}$

- if $f(U) \subset U$ for an open set $U$ whose complement (in $\overline{\mathbb{C}}$ ) consists of at least three points, then $U \subset F(f)$ (Montel).

Here and in the following, for proofs see [5], [6] or [12]. The classical references are [22], [23], [24], [25], [32] and [33].

A maximal domain of normality of the iterates of $f$ is a component of the Fatou set. The image of a component $U$ is always contained in a component $U_{1}$, and a component $U$ is called periodic of period $p$ if $f^{p}(U) \subset U$ and $p$ is the smallest integer having this property.

For entire functions, an invariant Fatou component $U$ satisfies one of the following:

- $U$ contains an attracting fixed point $z_{0}$. Then $f^{n}(z) \rightarrow z_{0}$ for every $z \in U$ as $n \rightarrow \infty$ and $U$ is called the immediate attracting basin of $z_{0}$. $A=\bigcup_{n=0}^{\infty} f^{-n}(U)$ is called the attracting basin of $z_{0}$.

- $\partial U$ contains a fixed point $z_{0} \in \mathbb{C}$ and $f^{n}(z) \rightarrow z_{0}$ for every $z \in U$ as $n \rightarrow \infty$. Then $f^{\prime}\left(z_{0}\right)=e^{2 \pi i p / q}$ for some $p / q \in \mathbb{Q}$. In this case, $U$ is called parabolic domain or Leau domain.

- There exists an analytic homeomeorphism $\Phi: U \rightarrow \mathbb{D}$, where $\mathbb{D}$ is the unit disk, such that $\Phi\left(f\left(\Phi^{-1}(z)\right)\right)=e^{2 \pi i \alpha}$ for some $\alpha \in \mathbb{R} \backslash \mathbb{Q}$. In this case, $U$ is called a Siegel disk.

- $f^{n}(z) \rightarrow \infty$ for every $z \in U$ as $n \rightarrow \infty$. In this case, $U$ is called a Baker domain.

The classification for periodic components is analogous.

If $f^{n}(U) \cap f^{m}(U)=\emptyset$ for a component $U$ and all $n \neq m \in \mathbb{N}$, then $U$ is called a wandering domain. A crucial role in iteration theory is played by the singularities of the inverse function. Let $C P(f)$ be the set of all roots of $f^{\prime}$, called critical points, $C V(f)=f(C P(f))$ the set of critical values of $f$ and $A V(f)$ be the set of asymptotic values, these are all values $v$ such there exists a continuous curve $\gamma$ tending to $\infty$ such that $f(z) \rightarrow v$ as $z \in \gamma$ and $|z| \rightarrow \infty$. The points where not all branches of the inverse function of $f$ can be defined holomorphically are 
- all critical values

- all asymptotic values and

- limit points of critical or asymptotic values.

These points form the set of singular values $S V(f)$. The last possibility can only occur if the function $f$ has infinitely many asymptotic values. We define the set of post-singular values (the post-singular set) by $P(f)=\bigcup_{n=0}^{\infty} f^{n}(S V(f))$. We have the following relations between the postsingular set and the components of the Fatou set:

\section{Theorem}

Let $C=\left\{U_{0}, \ldots, U_{p-1}\right\}$ be a periodic cycle of Fatou components.

a)

If $C$ is a cycle of immediate attracting basins or Leau domains, then

b) $U_{j} \cap S V(f) \neq \emptyset$ for some $j \in\{0, \ldots, p-1\}$.

If $C$ is a cycle of Siegel disks, then $\partial U_{j} \subset \overline{P(f)}$ for all $j \in\{0, \ldots, p-1\}$.

Concerning the Cremer points, the following holds:

Theorem (Zheng, [55])

If $z$ is a Cremer point of an entire function $f$, then $z$ is contained in the set of accumulation points of $P(f)$, called the derived set $P(f)^{\prime}$ of $P(f)$.

The relations between wandering domains or Baker domains and the postsingular values are more complicated, we will give some results in the next section.

A result on the connectivity of Fatou components we will use is the following one:

\section{Theorem}

a)

All periodic components of the Fatou set of $f$ are simply connected.

b)

If $F(f)$ contains a Baker domain, then all components of the Fatou set are simply connected.

In the remainder of this section we will introduce some terms we will work with in the following chapters. 
Let $g$ be a holomorphic self-map of the punctured plane $\mathbb{C}^{*}$ such that $\exp \circ f=g \circ \exp$. Then $f$ is called a logarithmic lift of $g$, and we have $J(g) \backslash\{0\}=\exp (J(f))$. Furthermore, $z$ is a critical point of $f$ if and only if $e^{z}$ is a critical point of $g$ (see [8]).

The following results and definitions are given in [13]:

Let $f$ be an entire transcendental function. Let $D$ be an open disk in the plane which contains all singular values of $f$ and let $\Sigma$ be the complement of $D$. Then any connected compent $T$ of $f^{-1}(\Sigma)$ is an unbounded disk and $f: T \rightarrow \Sigma$ is a universal covering. We call an invariant subset $C$ of $J(f)$ an $N$-bouquet for $f$ if for $\Sigma_{N}=\left\{(s)=\left(s_{0} s_{1} s_{2} \ldots\right)\left|s_{j} \in \mathbb{Z},\right| s_{j} \mid \leq N\right\}$ and $N \in \mathbb{N}$ the following holds:

- there exists a homeomorphism $h: \Sigma_{N} \times[0, \infty) \rightarrow C$

- $\pi \circ h^{-1} \circ f \circ h(s, t)=\sigma(s)$, where $\pi: \Sigma_{N} \times(0, \infty) \rightarrow \Sigma_{N}$ is the projection map and $\sigma: \Sigma_{N} \rightarrow \Sigma_{N}$ is the shift map defined by $\sigma\left(s_{0}, s_{1}, s_{2}, \ldots\right)=\left(s_{1}, s_{2}, \ldots\right)$

- $\lim _{t \rightarrow \infty} h(s, t)=\infty$ for any $s \in \Sigma_{N}$

- $\lim _{n \rightarrow \infty} f^{n} \circ h(s, t)=\infty$ if $t \neq 0$.

Let $C_{n}$ be an $n$-bouquet and suppose that $C_{n} \subset C_{n+1} \subset \ldots$ is an increasing sequence of bouquets. Then the set $C=\overline{\cup_{n \geq 0} C_{n}}$ is called a Cantor bouquet.

We will use quasi-conformal maps in some proofs, we will just give the basic definitions. For more on quasi-conformal mappings see [1] or [39].

Definition (quasi-regular, quasi-conformal map)

$A$ continuous map $\phi$ is called $K$-quasi-regular $(K \geq 1)$ if locally it has distributional derivatives and the complex dilatation $\mu(z)$ defined locally by

$$
\frac{\partial \phi}{\partial \bar{z}}=\mu(z) \frac{\partial \phi}{\partial z}
$$

satisfies $|\mu| \leq \frac{K-1}{K+1}$ almost everywhere.

If $\phi$ is $K$-quasi-regular for some $K$, we say that $\phi$ is quasi-regular. If $\phi$ is $(K-)$ quasi-regular and injective, then $\phi$ is called $(K$-)quasi-conformal.

A quasi-regular map can always be written as a composition $f \circ \phi$ of a quasi-conformal map $\phi$ and a holomorphic map $f$. A map is analytic iff 
it is 1-quasi-regular.

A polynomial-like map of degree $d \geq 2$ is a triple $\left(f, U, U^{\prime}\right)$, where $U$ and $U^{\prime}$ are open sets in $\mathbb{C}$ isomorphic to discs with $\bar{U} \subset U^{\prime}$ and $f: U \rightarrow U^{\prime}$ is a holomorphic map such that every point in $U^{\prime}$ has exactly $d$ preimages counted with multiplicity.

The filled Julia set of a polynomial-like map $f$ can be defined in the same fashion as for polynomials by

$$
K_{f}=\left\{z \in U: f^{n}(z) \in U \text { for all } n \in \mathbb{N}\right\} .
$$

We say that two functions $f, g$ are quasi-conformally conjugate if $f \circ \phi=\phi \circ g$ for some quasi-conformal homeomorphism $\phi$. Two polynomiallike maps $f, g$ are hybrid equivalent if they are quasi-conformally conjugate and the conjugacy $\phi$ can be chosen such that $\bar{\partial}_{z} \phi=0$ almost everywhere on $K_{f}$.

The relation between polynomial-like maps and an actual polynomial is given in the Straightening Theorem ([16]):

Theorem (Douady, Hubbard)

Let $f: U \rightarrow U^{\prime}$ be a polynomial-like map of degree $d$. Then, $f$ is hybrid equivalent to a polynomial of degree $d$. If $K_{f}$ is connected, then $P$ is unique up to conjugation by an affine map.

Definition (analytic family)

Let $\Lambda$ be a Riemann surface and $\mathcal{F}=\left\{f_{\lambda}: U_{\lambda} \rightarrow U_{\lambda}^{\prime}, \lambda \in \Gamma\right\}$ be a family of polynomial-like maps. Set

$$
\begin{aligned}
U & =\left\{(z, \lambda): z \in U_{\lambda}\right\} \\
U^{\prime} & =\left\{(z, \lambda): z \in U_{\lambda}^{\prime}\right\} \\
f(\lambda, z) & =\left(\lambda, f_{\lambda}(z)\right) .
\end{aligned}
$$

Then, $\mathcal{F}$ is called an analytic family of polynomial-like maps if it satisfies the following properties:

- $U$ and $U^{\prime}$ are homeomorphic over $\Lambda$ to $\Lambda \times \mathbb{D}$

- the projection from the closure of $U$ in $U^{\prime}$ to $\Lambda$ is proper

- the map $f: U \rightarrow U^{\prime}$ is holomorphic and proper. 
We can define the set

$$
M_{\mathcal{F}}=\left\{\lambda \in \Lambda: K_{\lambda} \text { is connected }\right\}
$$

in analogy to the Mandelbrot set $\mathcal{M}$.

Let $\mathcal{F}=\left\{f_{\lambda}: U_{\lambda} \rightarrow U_{\lambda}^{\prime}, \lambda \in \Gamma\right\}$ be an analytic family of polynomial-like maps of degree 2. For each $\lambda \in M_{\mathcal{F}}, f_{\lambda}$ is hybrid equivalent to a quadratic polynomial of the form $Q_{c}(z)=z^{2}+c$. The map

$$
\begin{aligned}
C: \quad M_{\mathcal{F}} & \rightarrow \mathcal{M} \\
\lambda & \mapsto c=C(\lambda)
\end{aligned}
$$

is well-defined. We have the theorem

Theorem (Douady, Hubbard)

Let $A \subset \Lambda$ be a closed set of parameters homeomorphic to a disk and containing $M_{\mathcal{F}}$, $w_{\lambda}$ the critical point of $f_{\lambda}$ and suppose that for each value $\lambda \in \Lambda \backslash A$ the critical value $f_{\lambda}\left(w_{\lambda}\right) \in U_{\lambda}^{\prime} \backslash U_{\lambda}$. Assume also that as $\lambda$ goes once around $\partial A$, the vector $f_{\lambda}\left(w_{\lambda}\right)-w_{\lambda}$ turns once around the origin. Then, the map $C$ is a homeomorphism and it is analytic in the interior of $M_{\mathcal{F}}$.

A family $\mathcal{F}$ satisfying the hypothesis of this theorem is called Mandelbrot-like.

The proof of the following powerful tool can be found in [26], [29] or [53].

Lemma (generalized qc-Lemma)

Let $f: \mathbb{C} \rightarrow \mathbb{C}$ be a quasi-regular map. Assume that all iterates $f^{n}$ are $K$-quasi-regular with a fixed $K$. Then there exists a quasi-conformal map $\phi: \mathbb{C} \rightarrow \mathbb{C}$ such that $\phi \circ f \circ \phi^{-1}$ is analytic.

\subsection{Baker domains and wandering domains}

In this section we will give a short survey on Baker domains.

The first example of a Baker domain was given by Fatou in 1919, his example was the function $f(z)=z-1+e^{z}$ (with minor changes in notation). The left half plane is invariant with respect to $f$, and it is easy to see that the sequence of iterates of $f$ tends to the constant function $\infty$ on this half plane. Hence, there is a Baker domain containing the left half plane. It also contains all (infinitely many) singularities of the inverse function.

A significantly different example was given by Herman [28] in 1985, the function $f(z)=z+i \lambda+e^{-z}$ has for a suitable value of $\lambda \in \mathbb{R}$ a Baker domain 
again containing a half plane. This Baker domain does not contain singular values.

Another example of a univalent Baker domain was given by Bergweiler ([7], 1995), he showed that the function $f(z)=2 z+\log 2-2+e^{-z}$ also features a univalent Baker domain.

In 1997, Baker and Dominguez [2] showed that the Fatou set of $f(z)=z+e^{-z}$ contains infinitely many Baker domains, each of them is contained in a strip of height $2 \pi$. Each of these Baker domains contains exactly one singular value.

All of these examples have in common that the functions $f$ are logarithmic lifts of entire functions.

Examples of Baker domains not obtained by logarithmic lifts were given by Rippon and Stallard in 1999 ([48] and [49]), they showed that for

$a>1, b>0, k=0,1, \ldots$ the function $f(z)=a z+b z^{k} e^{-z}(1+o(1))$ has a Baker domain containing a large part of the right half plane, if $a=1$ then there are infinitely many Baker domains and each of them is contained in a horizontal strip.

The same authors also give functions having one or infinitely many $p$-cycles of Baker domains for any $p \in \mathbb{N}$. They show that in some of these examples, $f$ is univalent on such a $p$-cycle, while in other examples there are $p$-cycles containing infinitely many critical values.

In 2001, Baranski and Fagella [3] proved the existence of an invariant, univalent Baker domain which is approximately a logarithmic spiral.

Now we turn to general theorems on Baker domains. As mentioned in the introduction, Baker domains of entire functions are necessarily simply connected.

The first result on relations between the set of singular values and Baker domains was given by Eremenko and Lyubich [19] who proved that if an entire function has a Baker domain, then its set of singular values is unbounded. Herman observed that one can use Sullivan's idea in the nowandering-domains-theorem for rational functions to show further connections, for example, that any cycle of Baker domains of $f(z)=z+P(z) e^{Q(z)}$, where $P$ and $Q$ are polynomials, meets $S V(f)$.

The result of Eremenko and Lyubich was strengthened by Bargmann ([4], 2001), who proved that if an entire function has an invariant Baker domain, then there exist constants $c>1$ and $r_{0}>0$ such that

$S V(f) \cap\{r<|z|<c r\} \neq \emptyset$ for all $r>r_{0}$. On the other hand, there are many examples of Baker domains not containing singular values. The example of Bergweiler mentioned earlier even features a Baker domain $U$ of an entire function $f$ such that the distance between $U$ and the postsingular set is strictly positive. Bergweiler gives in [10] some necessary conditions for the 
existence of univalent Baker domains.

Finally, Bergweiler showed in the same paper that if $a, \alpha>0$, $f(z)=z+a+o(1)$ as $z \rightarrow \infty$ and $|\arg z| \leq \alpha$ and $f$ is of finite order, then $f$ has a Baker domain $U$ containing a sector of the form $\{|\arg z| \leq \alpha, \operatorname{Re} z>R\}$ and $U \cap S V(f)$ is unbounded.

Baker and Dominguez [2] proved statements on the boundary of unbounded components of the Fatou set, in particular, that if $U$ is a non-univalent Baker domain of an entire transcendental function $f$, then the boundary of $U$ is highly disconnected and $U$ has infinitely many accesses to $\infty$.

Baranski and Fagella [3] gave a classification of univalent Baker domains. For a univalent Baker domain, the boundary can consist of one or two components, the question if it can consist of more than two components is still open.

A complete classification of Baker domains was given by König ([34], English version in [35]), we only cite the part concerning entire functions:

Definition (absorbing domain, conformal conjugacy)

Let $B$ be a Baker domain of the entire function $f$.

a)

$A$ domain $D \subset B$ is called absorbing for $f$ if

- $D$ is simply connected

- $f(D) \subset D$

- for all compact sets $K \subset B$ there exists an $n \in \mathbb{N}$ such that $f^{n}(K) \subset D$.

b)

Let $\mathbb{H}$ be the right half plane. A triple $(D, \phi, T)$ is called a conformal conjugacy if

- $D \subset B$ is absorbing for $f$

- $\phi: B \rightarrow \Omega \in\{\mathbb{H}, \mathbb{C}\}$ is analytic on $B$ and univalent on $D$

- $T: \Omega \leftrightarrow \Omega$ is a Möbius transformation, $\phi(D)$ is absorbing for $T$

- for all $z \in B$ we have $\Phi(f(z))=T(\Phi(z))$. 
Theorem (König)

Let $f$ be an entire transcendental function and $U_{0}, \ldots, U_{p-1}$ a $p$-cycle of Baker domains. Then there exists a conformal conjugation of $f^{p}$ in $U_{0}$. We can achieve that

- $T(z)=z+1$ and $\Omega=\mathbb{C}$ or

- $T(z)=z \pm i$ and $\Omega=\mathbb{H}$ or

- $T(z)=c z$ for some $c>1$ and $\Omega=\mathbb{H}$.

These possibilities exclude each other.

In other words, Baker domains are linearizable on a subset.

König also gave a criterion to check which case of the theorem applies, in the following theorem, " $f \sim z+1$ "refers to the first case in the preceding theorem, etc..

Theorem (König)

Let $B \subset \mathbb{C}$ be a hyperbolic domain, $f: B \rightarrow B$ be a holomorphic function with $f^{n} \rightarrow \infty$ for $n \rightarrow \infty$, and $(V, \phi, T)$ be a conformal conjugacy of $f$. For $w_{0} \in B$ set $w_{n}=f^{n}\left(w_{0}\right), d_{n}=d\left(w_{n}, \partial B\right)$. Then

$$
\begin{aligned}
& f \sim z+1 \quad \Leftrightarrow \lim _{n \rightarrow \infty} \frac{w_{n+1}-w_{n}}{d_{n}}=0 \text { for all } w_{0} \in B \\
& f \sim z \pm i \quad \Leftrightarrow \lim _{n \rightarrow \infty} \inf \frac{\left|w_{n+1}-w_{n}\right|}{d_{n}}>0 \text { for all } w_{0} \in B \\
& \text { but } \inf _{w_{0} \in B} \lim _{n \rightarrow \infty} \frac{w_{n+1}-w_{n}}{d_{n}}=0 \\
& f \sim c z \text { for some } c>1 \Leftrightarrow \lim _{n \rightarrow \infty} \frac{\left|w_{n+1}-w_{n}\right|}{d_{n}}>\beta \text { for all } w_{0} \in B, n \in \mathbb{N} \\
& \text { and some } \beta=\beta(f)>0 \text {. }
\end{aligned}
$$

König shows that in the first case of his theorem the Baker domain always contains a singular value.

Among various estimations for dynamics on Baker domains to be found in the papers of Rippon and Stallard and in [6] we only mention the following which implies some kind of "speed limit" on Baker domains: 
Lemma (Bergweiler, Rippon, Stallard)

If $B$ is an invariant Baker domain of the entire function $f$, then

a)

there exist a constant $A>1$ and a path $\gamma$ tending to $\infty$ such that $f(\gamma) \subset \gamma \subset B$ and $\frac{|z|}{A} \leq|f(z)| \leq A|z|$ for all $z \in \gamma$ and

b)

for any compact set $K \subset B$ there exists an $C>1$ such that $\left|f^{n}\left(z^{\prime}\right)\right|<C\left|f^{n}(z)\right|$ for all $n \in \mathbb{N}$ and $z, z^{\prime} \in K$.

Hence, the convergence to $\infty$ on Baker domains is always quite slow, at most of linear growth.

Since we will deal with some functions having wandering domains as well, we recall some facts on wandering domains.

First of all, the result of Eremenko and Lyubich also states that functions with a bounded set of singular values do not have wandering domains, neither. Fatou and Cremer proved that all limit functions on wandering domains are constant. Clearly, all finite limit functions are contained in the Julia set. This result was strengthened by Bergweiler, Haruta, Kriete, Meier and Terglane [9], who showed that the set of limit functions is contained in the set $P(f)^{\prime} \cup\{\infty\}$. A further known result is that if an entire function has a wandering domain, then either the constant function $\infty$ is the only limit function or there are infinitely many limit functions (this result can be obtained by combining the results of Perez-Marco [47] and Bueger [11], it was stated for the first time in [55]). In the first case, the wandering domain is called a Baker wandering domain. Eremenko and Lyubich proved in [18] that wandering domains of the second type exist, but no function having this phenomenon is explicitly known. Also open is the question if the set of finite limit function is always unbounded in this case.

Baker showed that in Baker wandering domains there are no limitations of the speed of convergence towards $\infty$, in contrary to the dynamics on Baker domains, as we have seen above.

There are plenty of examples of wandering domains, free of singular values or not, or with any given connectivity. But it is not known if it is possible to have a wandering domain $U$ of an entire transcendental function such that the distance between the forward iterates of $U$ and the postcritical set is strictly positive, as is possible for Baker domains (obviously, wandering domains having this property are Baker wandering domains). In section 3.1.1 we will use a necessary condition for this to happen. 
Finally, note the following definition:

Definition (asymptotic path)

Let $f$ be an entire transcendental function having wandering domains. We say that two wandering domains $V, W$ are equivalent if there exist $m, n \in \mathbb{N}$ such that $f^{m}(V)$ and $f^{n}(W)$ are contained in the same Fatou component. An equivalence class of this relation is called asymptotic path.

The term of asymptotic path is also referred to as "large orbit". 


\subsection{Notation}

Most of the notations we will use are standard notations, but some are not, so here is a list:

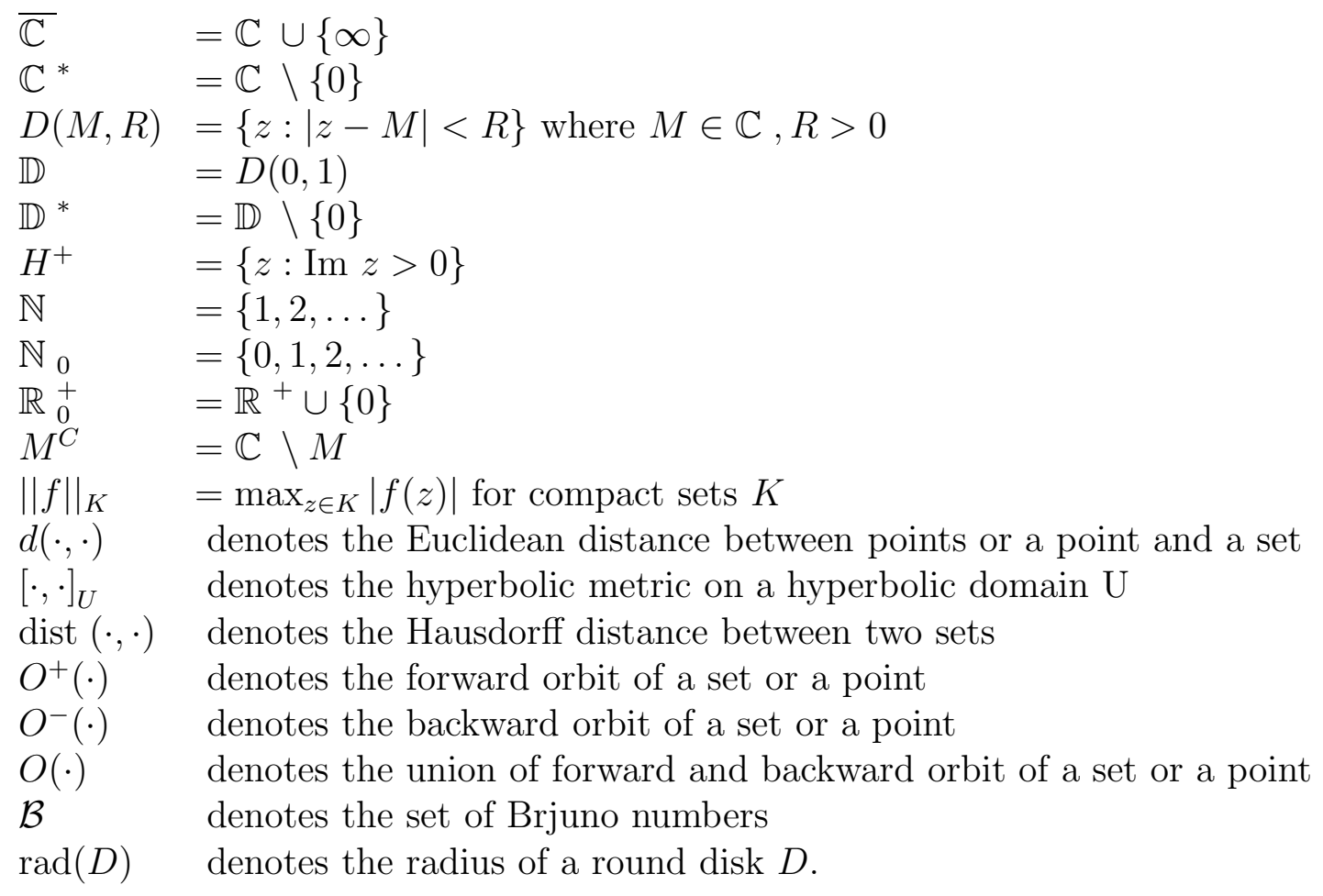




\section{Chapter 3}

\section{Results}

\subsection{A one-parameter family}

We will study the family of entire transcendental functions given by $\mathcal{F}_{b}=\left\{g_{b}: \mathbb{C} \rightarrow \mathbb{C}, g_{b}(z)=z-1+b z e^{z}\right\}$.

\subsubsection{The dynamical plane}

Fix a $b \in \mathbb{C}^{*}$.

\subsubsection{Existence of a Baker domain}

\section{Theorem 1}

The Fatou set of $g_{b}$ contains an invariant Baker domain $B_{b}$. For $\delta \in(0,1)$ and a sufficiently large $R>0$ the set $\left\{z:\left|b z e^{z}\right|<\delta\right.$, Re $\left.z<-R\right\} \subset B_{b}$ is an absorbing domain for $g_{b}$.

Concerning the dynamics on the Baker domain we have the following

\section{Proposition 1}

a)

For all $z \in B_{b}, g_{b}^{n+1}(z)-\left(g_{b}^{n}(z)-1\right)$ tends to 0 as $n$ tends to $\infty$.

b)

For any compact set $K$ contained in the Baker domain $B_{b}$ the set

$\left\{\operatorname{Im} z: z \in \bigcup_{i=0}^{\infty} g_{b}^{i}(K)\right\}$ is bounded.

We also have absorbing domains in the form of sectors:

\section{Corollary 1}

For any sufficiently small $v \in \mathbb{R}^{-}$and $\alpha \in(0, \pi)$, the set $\{z:|\arg (z-v)-\pi|<\alpha\}$ is an absorbing domain of $g_{b}$. 


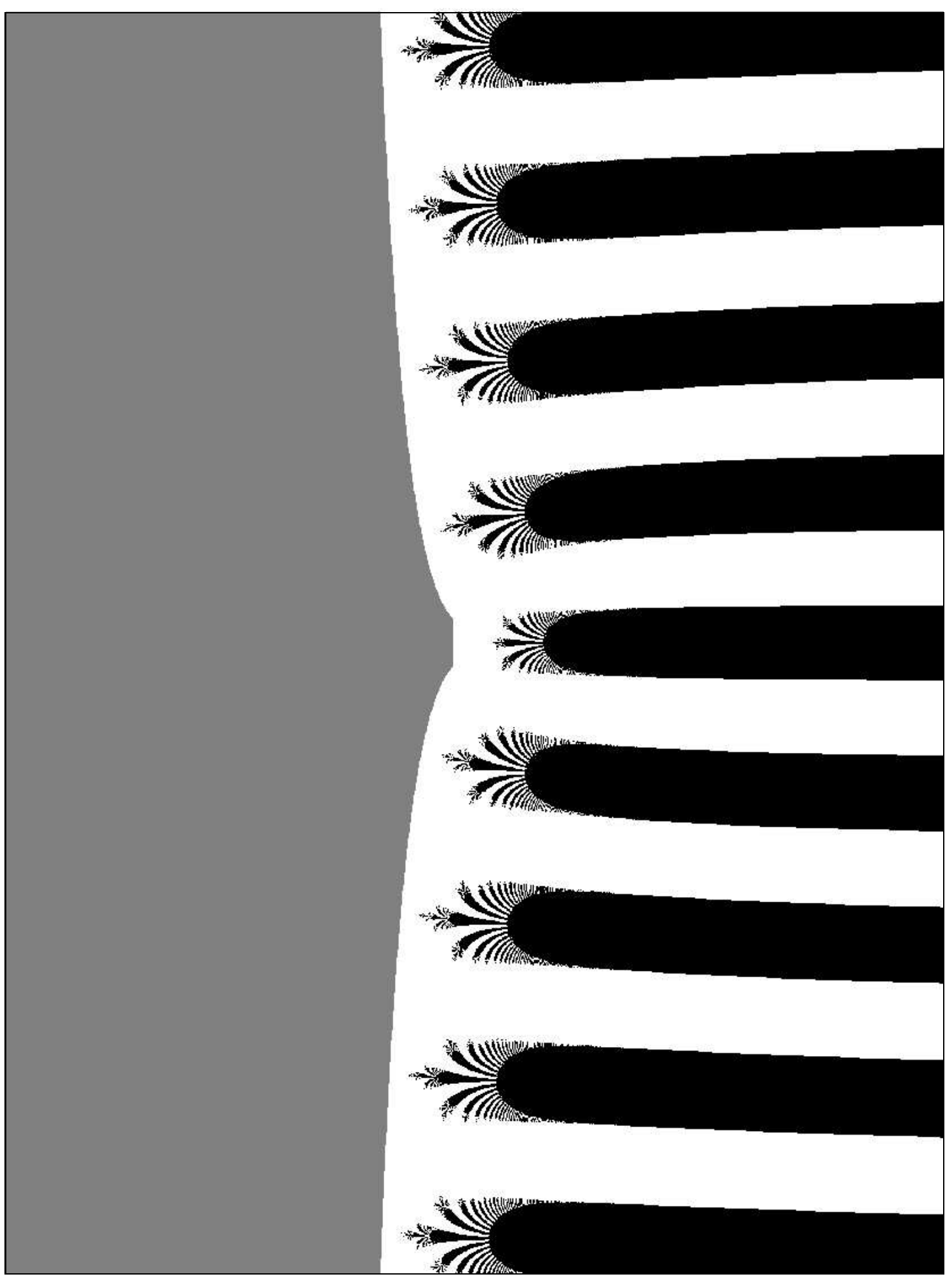

Figure 3.1: The Julia set of $g_{1}$ is shown in black, the grey set is the absorbing domain as given in the theorem with $\delta=0.5$ and $R=1$. 


\subsubsection{Singular values}

First, we state the following result on the type of singular values:

\section{Lemma 1}

$g_{b}$ has no asymptotic values and the set of critical values is unbounded.

Concerning the critical values, we have the following crucial result:

\section{Theorem 2}

$B_{b}$ contains all critical points of $g_{b}$ except for at most one.

This point, provided its existence, will be called the free critical point. We will see that we can find a function $b \mapsto z_{0}(b)$ holomorphic on $\mathbb{C} \backslash \mathbb{R}_{0}^{+}$, where $z_{0}(b)$ is the free critical point of $g_{b}$, provided its existence (if it does not exist, $z_{0}(b)$ is just some critical point contained in $B_{b}$ ). In the case $b \in \mathbb{R}^{+}$the free critical point does not exist (see the lemma below). In this sense, the free critical point moves "nicely", and the fact that we cannot define it for all parameters does not cause any inconveniences.

\section{Lemma 2}

If $b \in \mathbb{R}^{+}$, then all critical points of $g_{b}$ are contained in $B_{b}$.

\subsubsection{Components and geometry of the Fatou set}

An important tool will be the following proposition.

\section{Proposition 2}

For all bounded sets $M \subset \mathbb{C}$ there exists a domain $V \supset M$ such that the triple $\left(g_{b}, V, g_{b}(V)\right)$ is a polynomial-like map.

The number of additional periodic Fatou components is limited:

Proposition 3 (number of non-repelling cycles)

There exists at most one cycle of non-repelling periodic points of $g_{b}$ and $B_{b}$ is the only Baker domain in $F\left(g_{b}\right)$. Furthermore, all components of the Fatou set are simply connected and all (preimages of) attracting or parabolic domains or Siegel disks are bounded. 
The "skeleton" of the Fatou set is described as follows:

\section{Proposition 4}

i)

There are Jordan curves $\Gamma_{k}, k \in \mathbb{Z} \backslash\{0\}$, such that

- all $\Gamma_{k}$ are pairwise disjoint

- each $\Gamma_{k}$ is forward invariant and contained in $B_{b}$

- each $\Gamma_{k}$ contains exactly one critical point

- each $\Gamma_{k}$ is contained in a horizontal strip of height $5 \pi$

- each component $S$ of $\mathbb{C} \backslash \bigcup_{k \in \mathbb{Z} \backslash\{0\}} \Gamma_{k}$, except for at most one, is mapped biholomorphically to $\mathbb{C} \backslash\left(\gamma_{1} \cup \gamma_{2}\right)$, where $\gamma_{1}, \gamma_{2}$ are Jordan arcs contained in $\partial S_{k}$

- each $\Gamma_{k}$ stretches from "- $\infty$ " to " $\infty "$

- if $b \in \mathbb{C} \backslash \mathbb{R}_{0}^{+}$, then $\bigcup_{k \in \mathbb{Z} \backslash\{0\}} \Gamma_{k}$ contains all critical points of $g_{b}$ except for $z_{0}(b)$, if $b \in \mathbb{R}^{+}$, then all non-real critical points are contained in $\bigcup_{k \in \mathbb{Z} \backslash\{0\}} \Gamma_{k}$, so at most two are "missing".

ii)

$J\left(g_{b}\right)$ is thin at $\infty$, more exactly, there exists an $\varepsilon>0$ such that for all but finitely many $k \in \mathbb{Z} \backslash\{0\}$ the $\varepsilon$-neighborhood of $\Gamma_{k}$ is contained in $B_{b}$.

\section{Remark 1}

Using the same arguments as in [30] one can show that each component of $\mathbb{C} \backslash \bigcup_{k \in \mathbb{Z} \backslash\{0\}} \Gamma_{k}$ except for at most one contains a repelling fixed point located on the boundary of $B_{b}$. This point is also called Denjoy-Wolff point.

\section{Remark 2}

Using a theorem of Stallard in [52] it immediately follows that if the free critical point does not exist or is contained in the preimage of the Baker domain or attracted by an attracting cycle, then the Lebesgue measure of the Julia set of $g_{b}$ is equal to 0 . 


\subsubsection{On the existence of wandering domains}

The function $g_{b}$ has an infinite-dimensional Teichmüller space. Hence, we cannot follow Sullivan's idea to prove the non-existence of wandering domains, but we can give some criterions to rule out their existence.

\section{Theorem 3}

If $g_{b}$ has a wandering domain $V$, then

- there are no non-repelling periodic points

- the sequence of iterates of $g_{b}$ has infinitely many limit functions on $V$, including $\infty$

- the free critical point exists and is contained in the Julia set, but not in the boundary of a wandering domain (in particular, it is not contained in a wandering domain!). The sequence of its iterates has infinitely many accumulation points, including $\infty$

- $b \notin \mathbb{R}$.

In particular, $g_{b}$ has no Baker wandering domains. 


\subsubsection{The parameter plane}

Let $B\left(\mathcal{F}_{b}\right)=\left\{z\right.$ : all critical points of $g_{b}$ are contained in $\left.B_{b}\right\}$.

\section{Proposition 5}

$B\left(\mathcal{F}_{b}\right)$ is open, and all components of $B\left(\mathcal{F}_{b}\right) \cup\{0\}$ are simply connected.

Having a result in the next section in mind, we prove

\section{Theorem 4}

The parameter plane of $\mathcal{F}_{b}$ contains a copy of the Mandelbrot set. The main cardiod contains all parameters corresponding to functions having attracting fixed points.

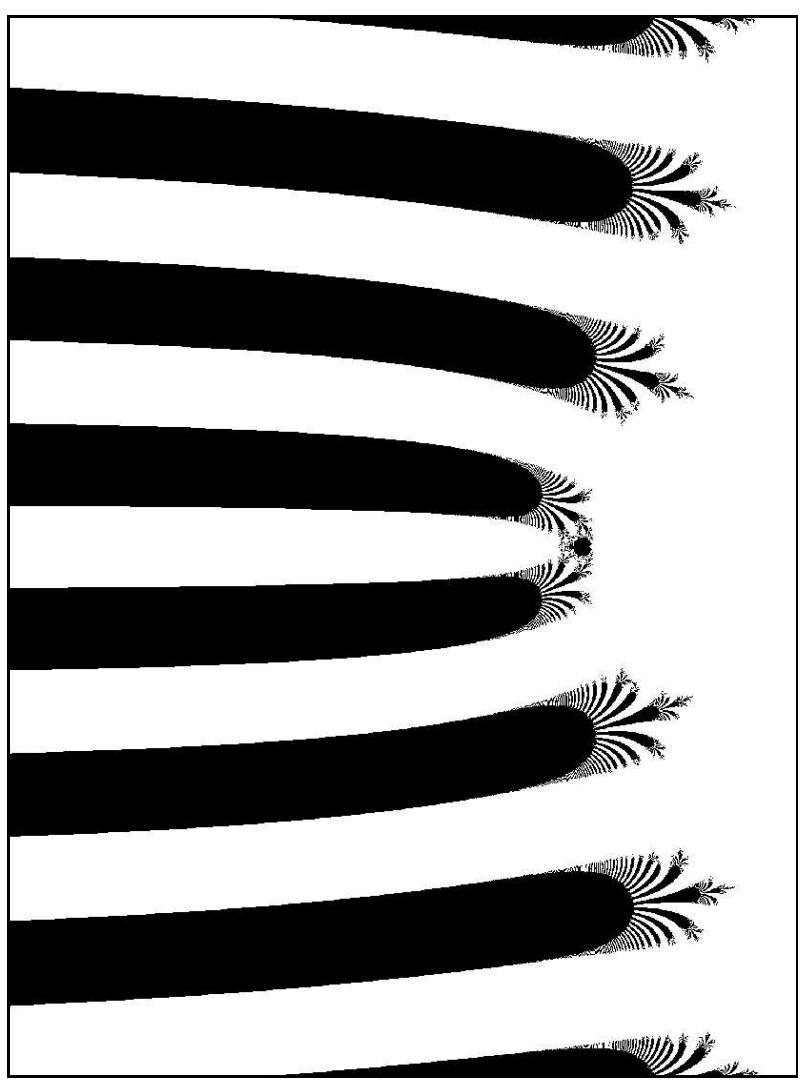

Figure 3.2: Parameter plane of $\mathcal{F}_{b}$, the set $B\left(\mathcal{F}_{b}\right)$ is shown in white.

Range: $[-57.5,17] \times[-50,50]$ 


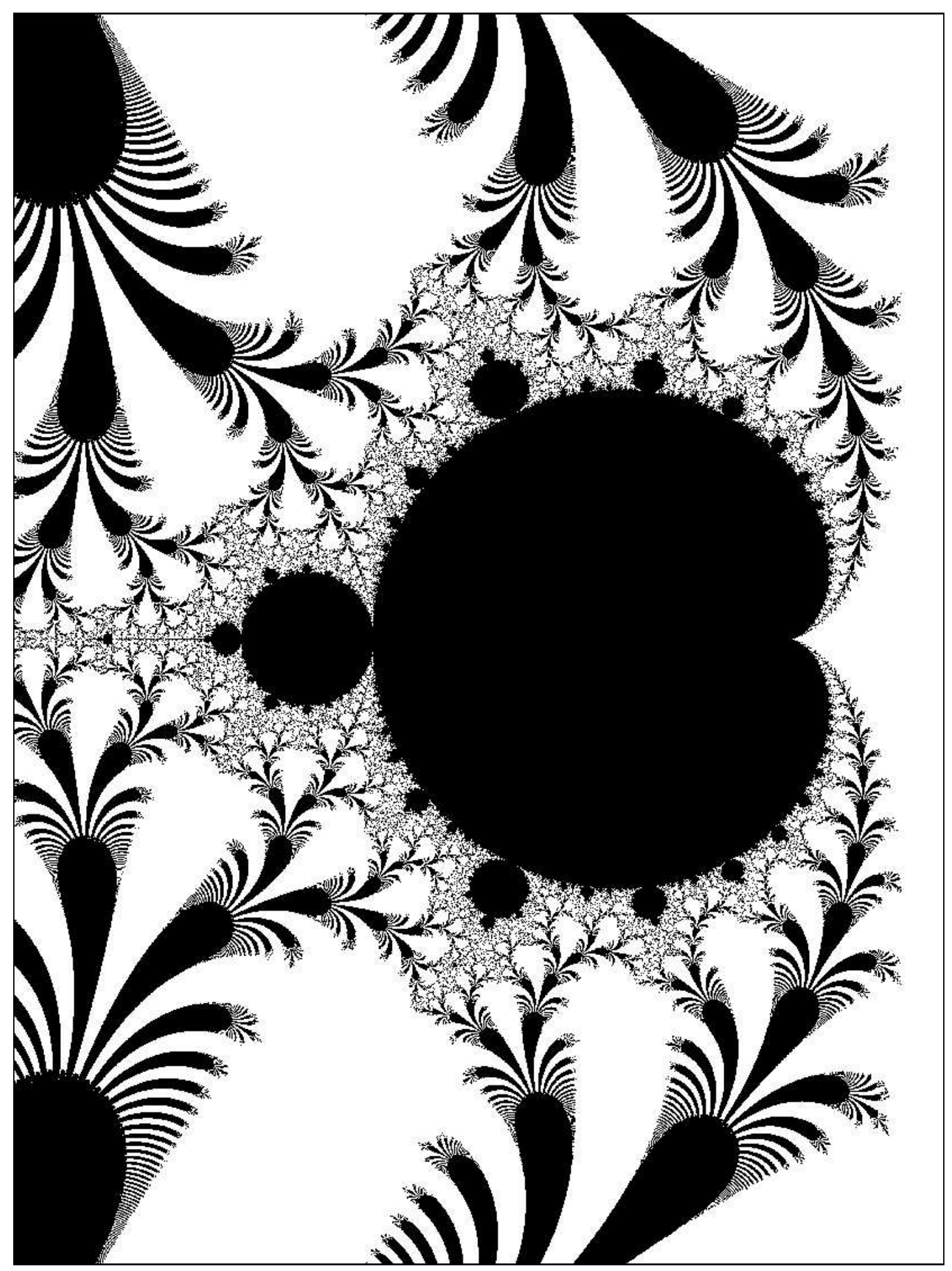

Figure 3.3: Zoom of the parameter plane of $\mathcal{F}_{b}$, range: $[-5.45,-2.15] \times[-2.2,2.2]$ 


\subsubsection{A related family}

Let $\mathcal{F}_{a}=\left\{f_{a}: \mathbb{C} \rightarrow \mathbb{C}, f_{a}(z)=z-1+(1-a z) e^{z}\right\}$. A short calculation shows that if $g_{b}(z)=z-1+b z e^{z}, f_{a}(z)=z-1+(1-a z) z e^{z}$ and $-a e^{\frac{1}{a}}=b$, then $g_{b}$ and $f_{a}$ are conjugated via the linear map $z \mapsto z+\frac{1}{a}$. Using this conjugacy we can immediately transfer the results on the dynamical plane of $\mathcal{F}_{b}$ to all functions in $\mathcal{F}_{a}$, except for the case $a=0$. In this case we have the following

Lemma $3(a=0)$

$f_{0}$ has no wandering domains, and the only periodic Fatou component is a Baker domain which contains all critical points.

0 is some kind of singularity in the parameter plane:

\section{Theorem 5}

In the parameter plane of $\mathcal{F}_{a}$ there exists a sequence of copies of the Mandelbrot set of period one (i.e. all parameters in the main cardiods correspond to functions having attracting fixed points) tending to the set $\{0\}$ in the Hausdorff metric. There also exists a 2:1-covering of the Mandelbrot set.

Let $B\left(\mathcal{F}_{a}\right)=\left\{z\right.$ : all critical points of $f_{a}$ are contained in the Baker domain of $\left.f_{a}\right\}$. This gives the next at first sight surprising result.

\section{Corollary 2}

$B\left(\mathcal{F}_{a}\right) \backslash\{0\}$ is open, but $B\left(\mathcal{F}_{a}\right)$ is not open.

An explanation to this phenomenon is the

\section{Lemma 4}

The set of critical points does not move continuously in 0 (with respect to the Euclidean metric).

More precisely, there are sequences $a_{n} \rightarrow 0$ such that $f_{a_{n}}$ has an "additional" critical point $z_{n}$ such that $\left|\operatorname{Re} z_{n}\right| \rightarrow \infty$, while the critical points of $f_{0}$ are contained in the imaginary axis. This additional critical point makes it possible that there are non-repelling cycles for $f_{a_{n}}$, while such cycles do not exist for $f_{0}$.

\section{Remark 3}

Using that the absorbing domains can be taken such that they move continuously one can show that if $f_{a}$ has no parabolic cycles, Siegel disks or wandering domains, then Julia set moves continuously in a with respect to 
the Hausdorff metric, even in $a=0$. On the other hand, there are parameters such that the Julia set does not move continuously there, as can be seen from the existence of Mandelbrot-like families and the discontinuities of the Julia set for the quadratic family.

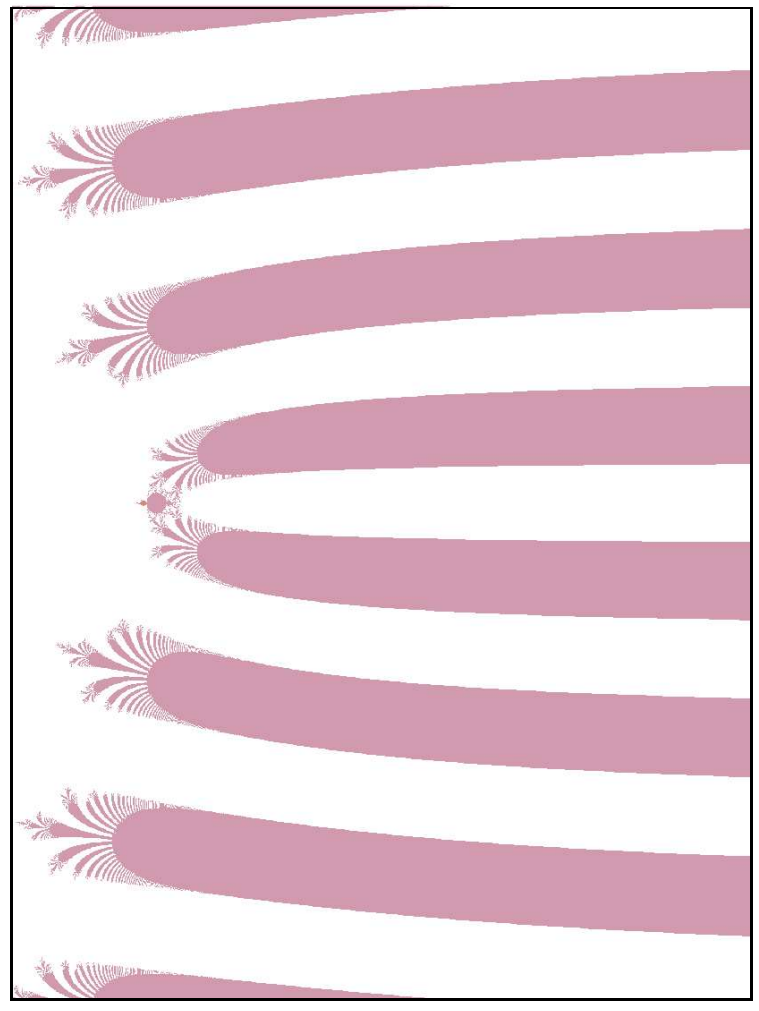

Figure 3.4: Parameter plane of $\mathcal{F}_{a}$, the set $B\left(\mathcal{F}_{a}\right)$ is shown in white. Range: $[-12.5,62.5] \times[-50,50]$

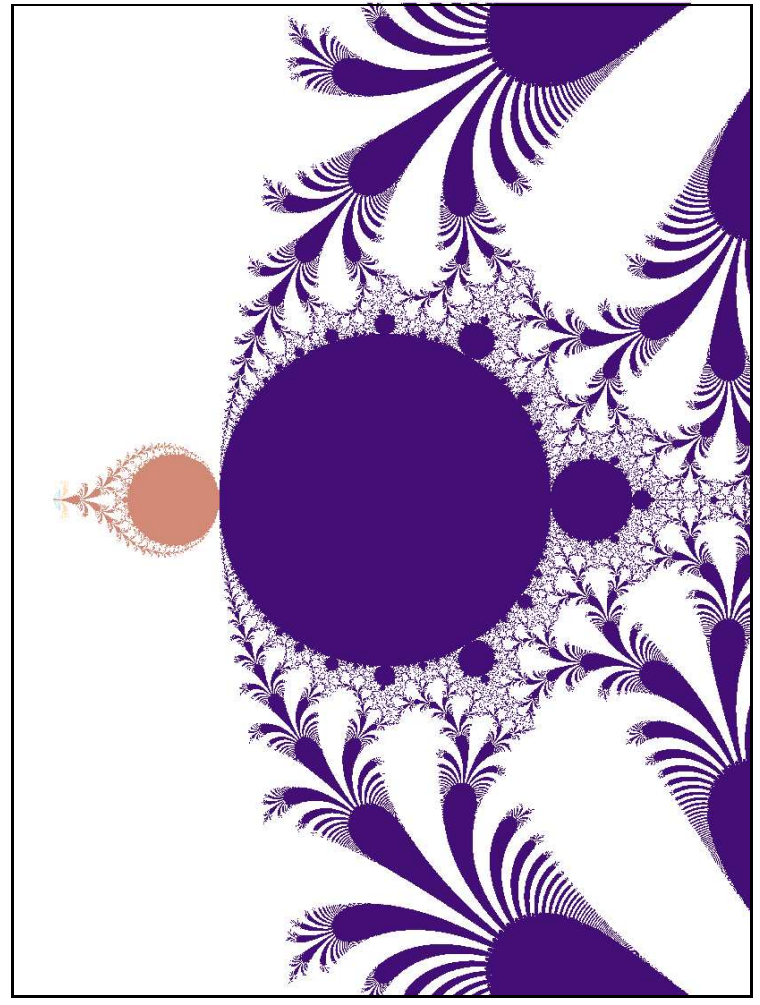

Figure 3.5: Zoom of the parameter plane of $\mathcal{F}_{a}$, range: $[-0.25,4.25] \times$ $[-3,3]$ 


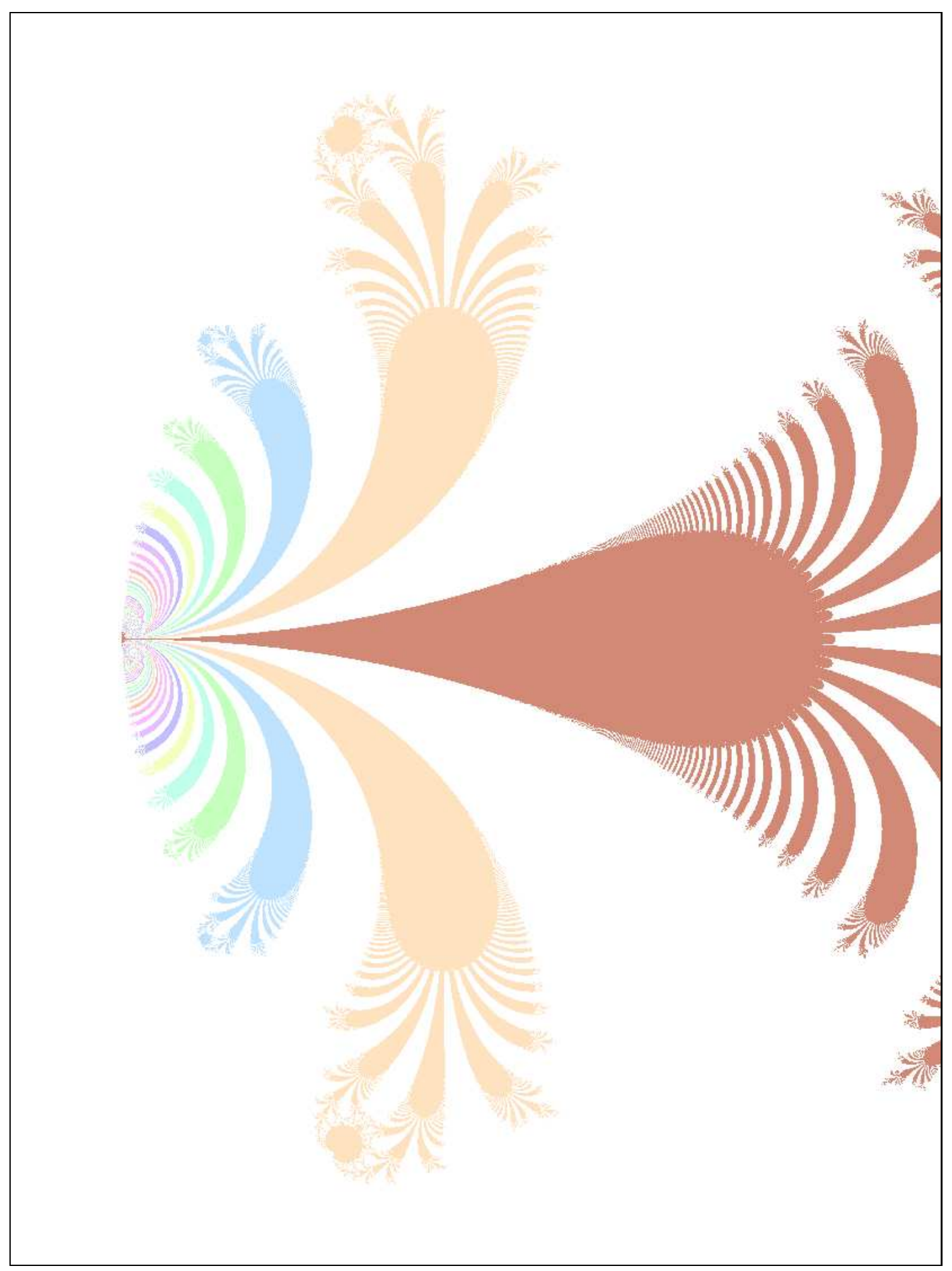

Figure 3.6: Zoom of the parameter plane of $\mathcal{F}_{a}$, range: $[-0.025,0.185] \times[-0.14,0.14]$ 


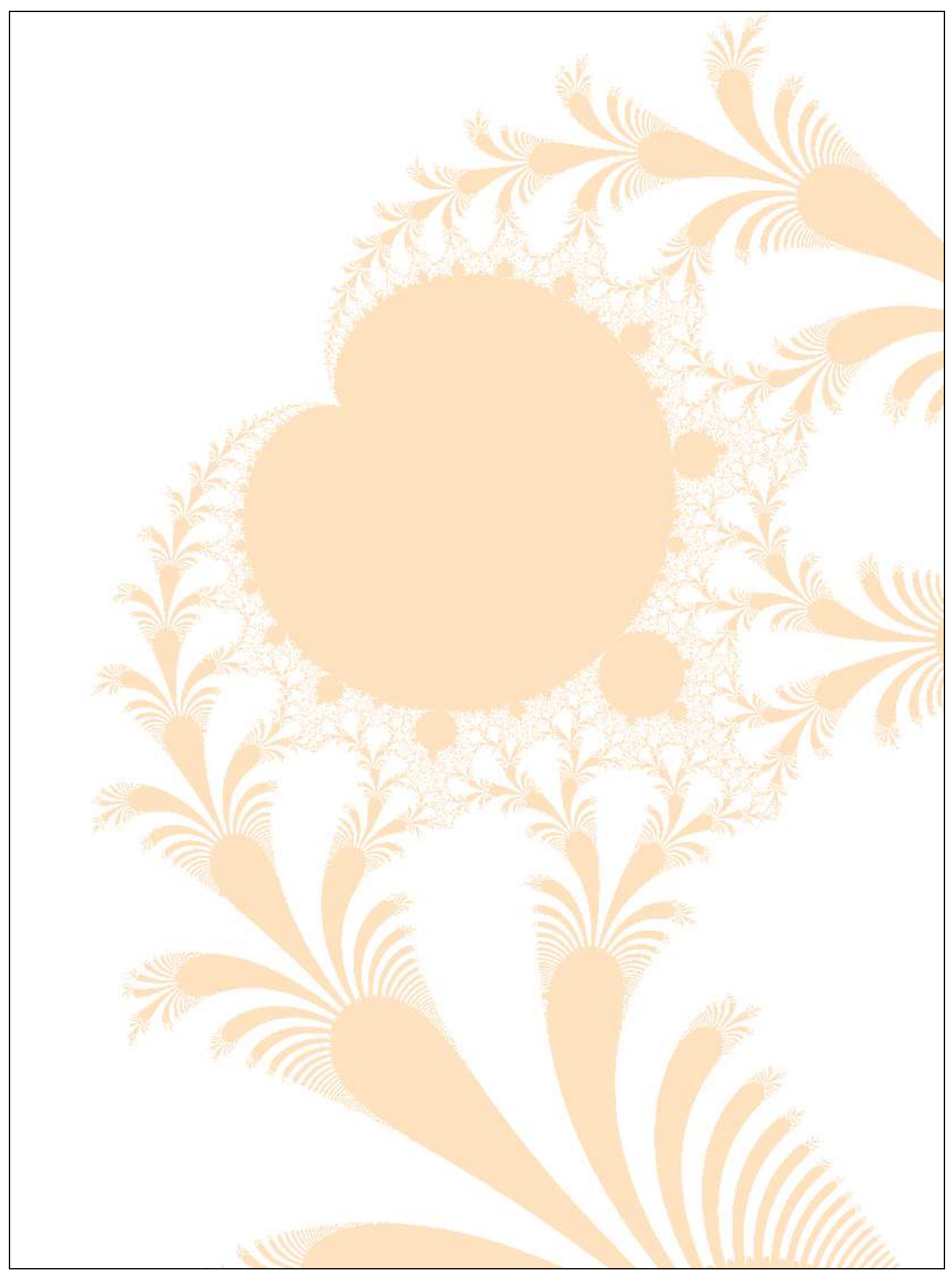

Figure 3.7: Zoom of the parameter plane of $\mathcal{F}_{a}$, range: $[0.041,0.059] \times[0.098,0.122]$ 


\subsection{Generalization}

Let $\mathcal{F}=\left\{f: \mathbb{C} \rightarrow \mathbb{C}: f(z)=z-c+P(z) e^{a z}, \operatorname{Re} a c>0,0 \not \equiv P\right.$ a polynomial $\}$. We will transfer some results from the preceding section to this more general class of functions and provide the properties we will need in the subsequent chapters.

A simple calculation gives

\section{Remark 4}

The class $\mathcal{F}$ is closed with respect to affine conjugations.

In the following, let $f \in \mathcal{F}$, so $f(z)=z-c+P(z) e^{a z}$ and $a, c, P$ as above.

\section{Lemma 5}

$f$ is a logarithmic lift of an entire function if and only if $P$ is constant and $a=1$.

We will take a closer look at functions obtained by logarithmic lifts in section 3.4.1. Concerning the singular values we have the following:

\section{Lemma 6}

$f$ has no asymptotic values.

Our previous methods are sufficient to establish the existence of Baker domains.

\section{Theorem 6}

The Fatou set of $f$ contains a Baker domain $B$, and for $\delta \in(0,1)$ and sufficiently large $R>0$ the set

$D_{\delta, R}=\left\{z \in \mathbb{C}:\left|P(z) e^{a z}\right|<\delta\right.$, Re az $\left.<-R\right\} \subset B$ is an absorbing domain for $f$. For all points $z \in B, f^{n+1}(z)-\left(f^{n}(z)-c\right) \rightarrow 0$ as $n$ tends to $\infty$.

\section{Corollary 3}

$B$ is an example of the first case in König's classification of Baker domains.

\section{Remark 5}

We can take $\delta$ and $R$ such that $D_{\delta, R}$ does not contain singular values.

If we restrict our class of functions, then we can make a statement on the number of free critical values.

\section{Proposition 6}

If Re ac $>0.3$, then $B$ contains all but finitely many critical values of $f$. 
This proposition reduces the number of possible periodic Fatou components.

\section{Lemma 7}

Let $h(z)=z-c$. There exists an absorbing domain $D$ of $B$ and a constant $M>0$ such that for all compact sets $K \subset D$ and all $n \in \mathbb{N}$ we have $d\left(f^{n}(K), h^{n}(K)\right)<M$.

These Baker domains have some features in common with invariant Leau domains; the same functional equation is satisfied (on the absorbing domains), and all points tend to $\infty$ along a unique attracting direction, which is illustrated by the following picture of the Julia set of $f(z)=z-1+e^{z}$ conjugated with $z \mapsto \frac{1}{z}$ :

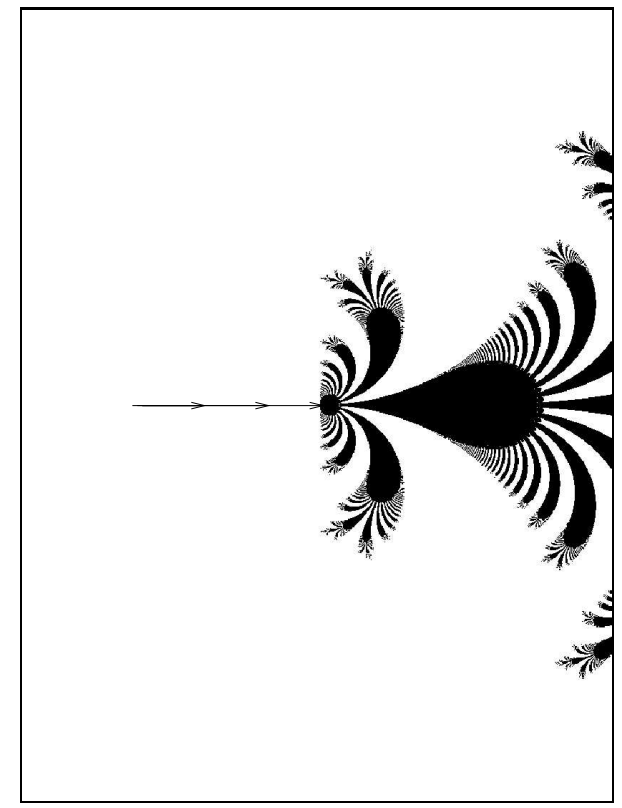

Figure 3.8:

Range: $[-0.2,0.2] \times[-0.3,0.3]$

\section{Remark 6}

In particular, if $a$ and $c$ are in $\mathbb{R}$, then for any compact set $K$ contained in the Baker domain the set $\left\{\operatorname{Im} z: z \in \bigcup_{i=0}^{\infty} f^{n}(K)\right\}$ is bounded.

Again, we can also find absorbing domains of a simpler form:

\section{Remark 7}

For any $\alpha \in(0, \pi)$ there exists a $m \in \mathbb{R}^{-}$such that the sector $\{z:|\arg (z-m)-\arg c|<\alpha\}$ is an absorbing domain. 


\section{Remark 8}

With some more technical work one may prove that for each $f \in \mathcal{F}$ there exist approximately horizontal channels contained in the Baker domain (as for the special case treated earlier). Having established this, the results on wandering domains and the existence of polynomial-like maps for $\mathcal{F}_{a}$ carry over to $\mathcal{F}$ (with some obvious changes), in particular, the absence of Baker wandering domains if $f(z)=z-c+P(z) e^{z}$ and Re $c>0.7$.

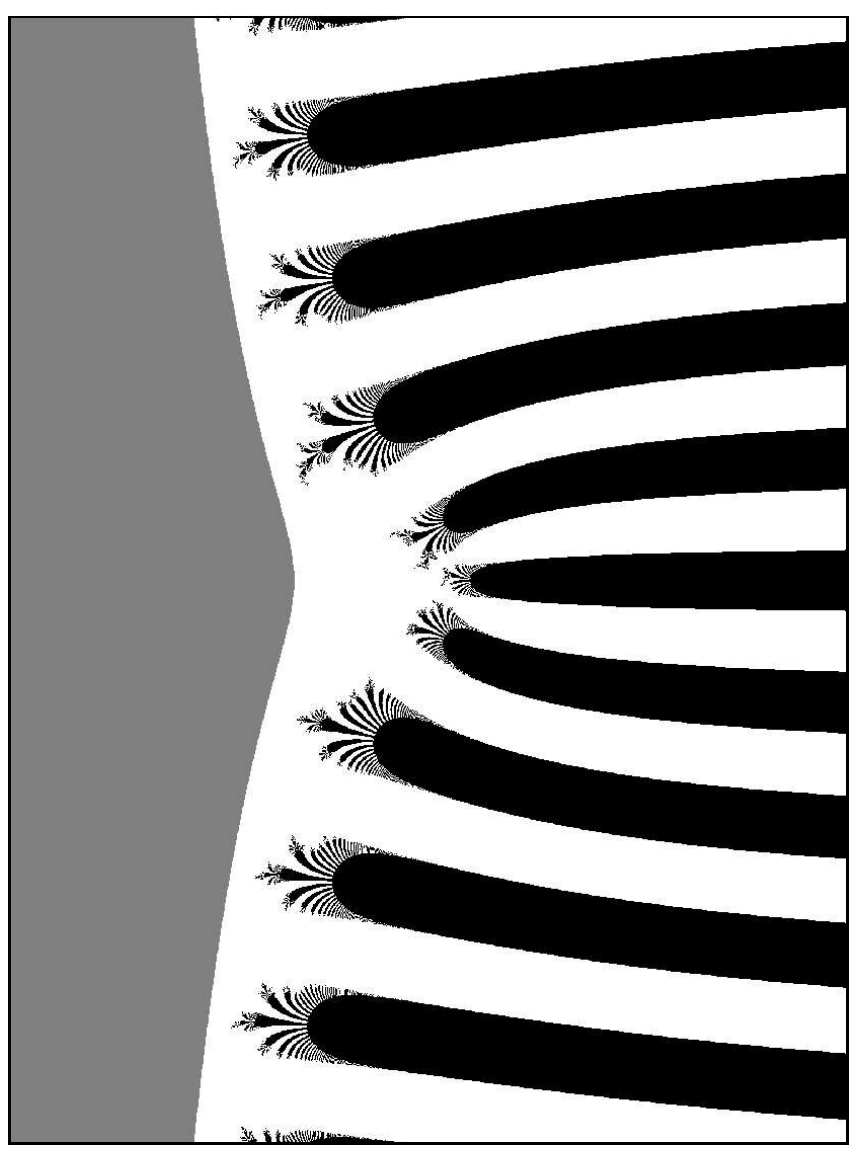

Figure 3.9: The Julia set of $f(z)=z-1-i+z^{3} e^{z}$ is shown in black, the grey set is the absorbing domain as given in the theorem with $\delta=0.5$ and $R=5$ 


\subsection{Approximation of Julia sets}

We will deal with the following problem: If we have a sequence of entire functions $f_{n}$ converging locally uniformly on compact subsets, do we have

$$
\lim _{n \rightarrow \infty} J\left(f_{n}\right)=J\left(\lim _{n \rightarrow \infty} f_{n}\right) ?
$$

In general, the answer is "no", as we will see below, but the question has been answered more detailed, depending on the type of Fatou components of the limit function.

\subsubsection{Preliminaries}

To talk about convergences, we need a metric. This will be provided in this section (cf. [42]).

Let $\rho$ be the chordal metric on $\overline{\mathbb{C}}$. The $\varepsilon$-neighborhood of a closed set $A$ with respect to the hyperbolic metric is denoted by $U_{\varepsilon}(A)$. Let $A$ and $B$ be non-empty compact sets in $\overline{\mathbb{C}}$. The Hausdorff distance dist $(A, B)$ is defined by

$$
\operatorname{dist}(A, B)=\inf \left\{\varepsilon>0: A \subset U_{\varepsilon}(B), B \subset U_{\varepsilon}(A)\right\} .
$$

This distance defines the Hausdorff metric on the set of all non-empty compact sets in $\overline{\mathbb{C}}$. Let $K$ and $K_{n}$ be non-empty compact sets in $\overline{\mathbb{C}}$. We say that $K_{n}$ converges to $K$ in the Hausdorff metric if dist $\left(K_{n}, K\right) \rightarrow 0$ as $n$ tends to $\infty$.

The following property of Julia is also referred to as lower semicontinuity. Henceforth, " converge" means "converge locally uniformly on compact subsets".

Lemma 8 (lower semicontinuity, [15],[42])

Let $f_{n}$ and $f$ be entire functions. If $f_{n}$ converges to $f$, then, for an arbitrary $\varepsilon>0$, there exists an $N>0$ such that

$$
J(f) \subset U_{\varepsilon}\left(J\left(f_{n}\right)\right)
$$

for all $n>N$.

The proof is based on the fact that the Julia set is equal to the set of repelling periodic points. Hence, if we take any $z \in J(f)$, we can find in a $\varepsilon / 2$-neighborhood of $z$ a repelling periodic point of $f$, by the Hurwitz theorem we then obtain that all but finitely many $f_{n}$ have repelling periodic points in the $\varepsilon$-neighborhood of $z$. 
Definition 1 (Carathéodory convergence of open sets)

Let $O$ and $O_{n}$ be open sets in $\overline{\mathbb{C}}$. We say that $O_{n}$ converges to $O$ in the Carathéodory sense if the following two conditions hold:

- for any compact set $I \subset O$ we have $I \subset O_{n}$ for all but finitely many $n \in \mathbb{N}$

- if an open set $U$ is contained in $O_{m}$ for infinitely many $m \in \mathbb{N}$, then $U \subset O$.

The two ideas of convergence defined above have the following relationship:

\section{Lemma 9}

Non-empty compact sets $K_{n}$ converge to $K$ in the Hausdorff metric if and only if the complements $K_{n}^{C}$ of $K_{n}$ converge to the complement $K^{C}$ of $K$ in the Carathéodory sense.

The lemma on semicontinuity is rephrased as follows:

\section{Lemma 10}

Assume that entire functions $f_{n}$ converge to $f$ locally uniformly. If for an open set $U U \subset F\left(f_{n}\right)$ holds for all but finitely many $n$, then $U \subset F(f)$.

Finally, we introduce a term which is slightly weaker than convergence in the Carathéodory sense:

Definition 2 (approaching of open sets)

Let $O$ and $O_{n}$ be open sets in $\overline{\mathbb{C}}$. We say that $O_{n}$ approach $O$ if

- for any compact set $I \subset O$ we have $I \subset O_{n}$ for all but finitely many $n \in \mathbb{N}$ and

- for any $\varepsilon>0, \partial O \subset U_{\varepsilon}\left(\partial O_{n}\right)$ for all but finitely many $n \in \mathbb{N}$.

An example of a sequence of Fatou components which approach an open set but do not converge to it are the basins of attraction of $\infty$ of the polynomials $Q_{n}(z)=z^{2}+0.25+\frac{1}{n}$ that approach the basin of $\infty$ of $Q_{0.25}(z)=z^{2}+0.25$, but do not converge to it. An example of Fatou sets of a sequence of functions that do not approach the Fatou set of the limit function is given by $f_{n}(z)=\left(\frac{1}{e}+\frac{1}{n}\right) e^{z}$, where $F\left(f_{n}\right)=\emptyset$ for all $n \in \mathbb{N}$, but $F\left(\lim _{n \rightarrow \infty} f_{n}\right)$ is not empty, since it contains a Leau domain. It is easy to check that we have the following criterion for approaching (forward and backward) orbits of Fatou components: 


\section{Lemma 11}

Assume that $f_{n}$ converges to $f$ locally uniformly and let $U$ be a Fatou component of $f$. If for any compact set $K \subset U$ we find an $m \in \mathbb{N}$ such that $f^{m}(K)$ is contained in Fatou components $U_{n}$ of $f_{n}$ for all but finitely many $n \in \mathbb{N}$, then $O\left(U_{n}\right)$ approaches $O(U)$. If all Fatou components of $f$ are approached by Fatou components of $f_{n}$, then $J\left(f_{n}\right) \rightarrow J(f)$ in the Hausdorff metric.

We remark that the set of all non-empty compact sets in $\overline{\mathbb{C}}$ is pre-compact (cf. [15]), so for any sequence of non-empty compact sets $K_{n}$ we find a converging subsequence.

\subsubsection{Known results}

We recall some known results on the continuity of Julia sets.

\section{Theorem 7}

If $f_{n}, n \in \mathbb{N}$, are entire functions converging to a function $f, \infty \in J(f)$ and $F(f)$ consists of attracting basins only, then $J\left(f_{n}\right)$ tends to $J(f)$ in the Hausdorff metric. In particular, this conclusion holds if $J(f)=\overline{\mathbb{C}}$.

The condition $\infty \in J(f)$ is necessary to avoid some pathological cases such as a sequence of entire transcendental functions tending to a polynomial.

\section{Remark 9}

In general, attracting basins of finite periodic points do not cause any discontinuities of Julia sets. If we have a sequence $f_{n}$ tending to $f$, where $F(f)$ contains an attracting basin $A$, then for sufficiently large $n$ the Fatou sets of the approximating functions contain attracting basins $A_{n}$ such that $\partial O\left(A_{n}\right)$ approaches $\partial O(A)$. The proof of this fact relies on Hurwitz' theorem stating that if $f$ has an attracting fixed point $z$ then all but finitely many of the approximating functions $f_{n}$ have attracting fixed points $z_{n}$ such that $z_{n} \rightarrow z$.

If we leave the smooth world of attracting cycles, we have to face more complicated situations, even if the functions we are dealing with are comparably simple: 
Theorem 8 (Douady, [15])

Let $P^{d}$ the set of polynomials of degree $d$.

a)

If $f_{0} \in P^{d}$ has a parabolic cycle, then the functions $f \mapsto J(f)$ and $f \mapsto K(f)$, where $K(f)$ is the filled Julia set of $f$, are not continuous at $f_{0}$ in $P^{d}$.

b)

If $f_{0} \in P^{d}$ has a Siegel disk but no parabolic cycle, then the function $f \mapsto J(f)$ is not continuous at $f_{0}$, but $f \mapsto K(f)$ is continuous at $f_{0}$ in $P^{d}$.

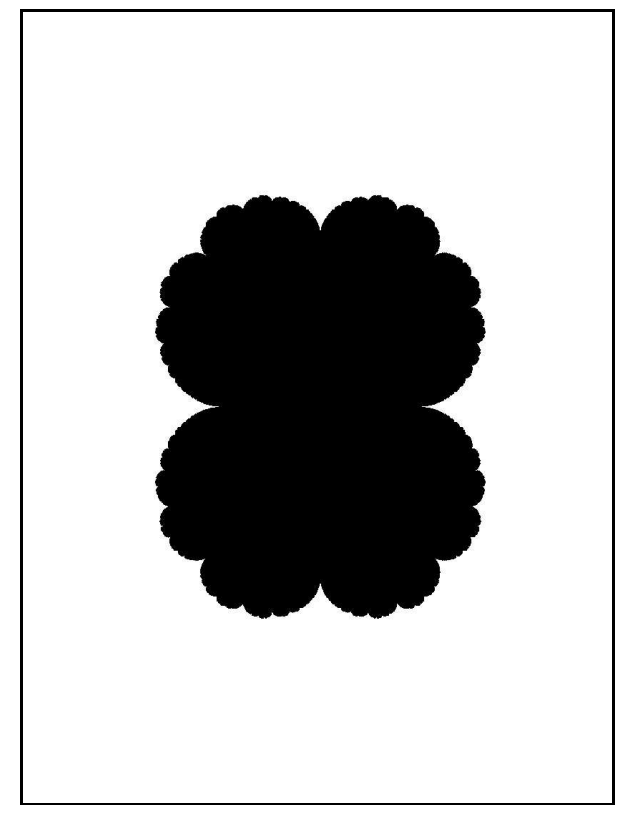

Figure 3.10: Filled Julia set of $Q_{0.25}(z)=z^{2}+0.25$

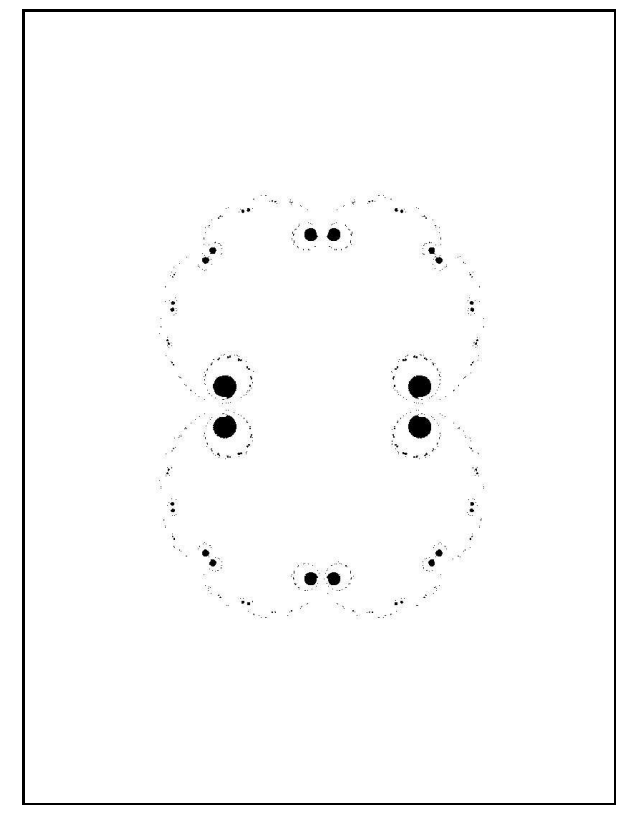

Figure 3.11: Filled Julia set of $Q_{0.25393+0.00046 i}(z)=z^{2}+0.25393+$ $0.00046 i$

Hence, the case of Siegel disks is "tame", while the case of parabolic cycles is not. This rich phenomenon is called "parabolic implosion", it also helped to better understand features of rational functions, for examples to prove that the Hausdorff dimension of the boundary of the Mandelbrot set is 2 or the existence of rational maps where the Hausdorff dimension of the Julia set is arbitrarily close to 2 . The parabolic implosion has been studied in detail (see ([15], [38], [45], [46] and [51]), it has been found that if the Fatou set of a rational function $f_{0}$ of degree $d$ consists of parabolic domains only (or their preimages), and $f_{n} \rightarrow f$ is a sequence of rational functions of degree $d$, then any possible limit set of the Julia sets of $f_{n}$ can be de- 
scribed in terms of a so-called Julia-Lavaurs set. This set is defined by $J\left(f_{0}, g\right)=\left\{z: g^{m}(z) \in J\left(f_{0}\right)\right.$ for some $\left.m \geq 0\right\}$, where $g$ is a holomorphic map defined in the parabolic basins. Some properties of these Julia-Lavaurs sets have been revealed, for example, they always have empty interior if $f_{0}$ is a polynomial.

One can say more if one restricts the set where the approximating functions come from. For example, it is known that if we take the quadratic polynomials $Q_{c}(z)=z^{2}+c$ and approach $Q_{1 / 4}$, then there are only finitely many directions in the $c$-plane causing discontinuities.

\subsubsection{Approximation of functions having Baker domains}

For Baker domains and wandering domains a corresponding theory has not been set up yet. Morosawa gave in [41] and [42] examples of Baker domains and wandering domains which are approximated by polynomials such that the Julia sets of the polynomials converge to the Julia set of the limit function in the Hausdorff metric. If we use the functions we considered in the last section, we will obtain the following:

\section{Theorem 9}

Let $P$ be a polynomial not vanishing everywhere, $a, c \in \mathbb{C}$ such that Re $a c>0$ and $f(z)=z-c+P(z) e^{a z}$. Let $B$ be the Baker domain of $f$ studied in 3.2.

a)

If $P_{n}(z)=\left(1-\frac{c}{n}\right) z-c+P(z)\left(1+\frac{a z}{n}\right)^{n}$, then there exist Fatou components $B_{n}$ of $P_{n}$ such that the $\partial O\left(B_{n}\right)$ approaches $\partial O(B)$. In particular, if $f$ has no wandering domains, Siegel disks or parabolic cycles and only one Baker domain, then $J\left(P_{n}\right)$ converges to $J(f)$ in the Hausdorff metric.

b)

If $Q_{n}(z)=z-c+P(z)\left(1+\frac{a z}{n}\right)^{n}$ and $a c \in[1,2)$, then $J\left(Q_{n}\right) \nrightarrow J(f)$ in the Hausdorff metric.

In general there may be discontinuities in case a) as well, but the Baker domain is not the "culpable" for that. The restriction of the values of $a c$ in b) is necessary for our proofs, since we use the fact that the polynomials $Q_{n}$ have attracting fixed points and that these basins intersect the Baker domain. If $a c \notin D(1,1)$, then $Q_{n}$ has no attracting fixed points anymore. 


\section{Remark 10}

Morosawa studied the sequence $\tilde{P}_{n}(z)=\left(1-\frac{1}{n}\right) z-1+\frac{n-1}{n+1}\left(1+\frac{z}{n}\right)^{n+1}$ and proved that the Julia sets of $\tilde{P}_{n}$ converge to the Julia set of $f(z)=z-1+e^{z}$ in the Hausdorff metric. Using symmetry arguments he showed that all critical points of $\tilde{P}_{n}$ tend to the same finite attracting fixed point, so the Julia set of $\tilde{P}_{n}$ is a simply closed curve, even a quasicircle. Fagella proved in [20] that the Julia sets of all $g_{\lambda}, \lambda \in \mathbb{C}^{*}$, contains Cantor Bouquets. Using the lift once more we obtain that the Julia set of the limit function $f$ contains a Cantor Bouquet, so we have the result that this very complicated structure in $J(f)$ is approximated by "smooth" curves!

The proofs of Morosawa can be generalized to all functions of the type $f(z)=z-c+e^{z}, c \in\{z: \operatorname{Re} z>0\}$ (if we set

$\left.\tilde{P}_{n}(z)=\left(1-\frac{c}{n}\right) z-c+\frac{n-c}{n+1}\left(1+\frac{z}{n}\right)^{n+1}\right)$, the result of Fagella gives us the existence of Cantor Bouquets for all of these functions as well. Further generalizations seem to be difficult, for one reason because the existence of Cantor Bouquets is more difficult to prove if $f$ is not a lift of an entire functions, for another reason, since the Fatou set of such an $f$ may contain several periodic Fatou components, so in general the Julia set of the approximating polynomials is not a simply closed curve (but still might be a closed curve).

One remaining question is how grave the discontinuity of the Julia set in part b) of the theorem is. The computer pictures seem to support the

\section{Conjecture 1}

$\partial \lim _{n \rightarrow \infty} K\left(P_{n}\right)=J(f)$.

If this was true we could speak, in analogy of the Siegel disk case mentioned in section 3.3.2, of "tame" discontinuities.

\section{Remark 11}

Numerical experiments suggest that the sequence of sets $J\left(Q_{n}\right)$ does not converge, but the sequences $J\left(Q_{2 n}\right)$ and $J\left(Q_{2 n+1}\right)$ do (to different limit sets). In the special case $Q_{n}(z)=z-1+\left(1+\frac{z}{n}\right)^{n}$ it is easy to verify that $-2 n$ is a super-attracting fixed point of $Q_{n}$ if $n$ is even, while for odd $n \mathbb{R}^{-}$belongs to the immediate basin of $\infty$.

\section{Remark 12}

The existence of parabolic periodic points for the limit function does not necessarily mean that the Julia sets of the polynomials do not converge. For example, if we look at the function $f_{a}(z)=z-1+(1-a z) e^{z}, a \in \mathbb{C}$, again, then we see that 0 is a parabolic periodic point of $f_{1}$. The polynomials $P_{n}(z)=\left(1-\frac{1}{n}\right) z-1+(1-z)\left(1+\frac{z}{n}\right)^{n}$ have attracting fixed points at 0 . 


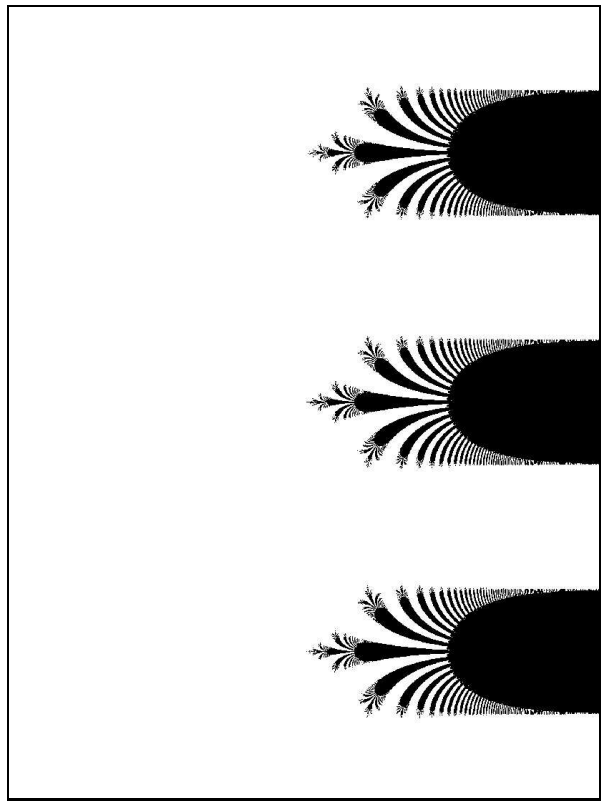

Figure 3.12: Julia set of $f(z)=z-1+e^{z}$, range: $[-7.5,7.5] \times[-10,10]$

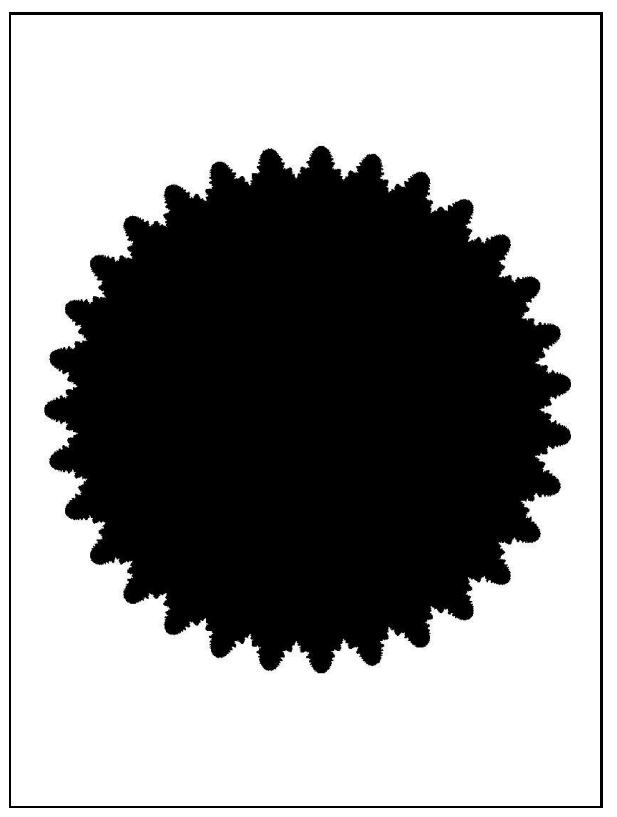

Figure 3.13: Filled Julia set of $P_{32}(z)=\left(1-\frac{1}{32}\right) z-1+\left(1+\frac{z}{32}\right)^{32}$, range: $[-71.75,-7.75] \times[-55,55]$

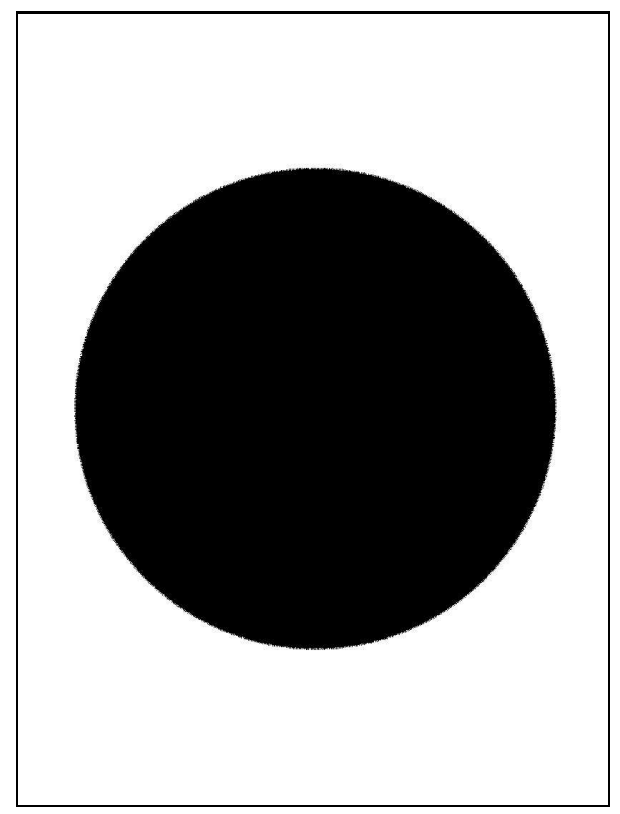

Figure 3.14: Filled Julia set of $P_{1024}(z)=\frac{1023}{1024} z-1+\left(1+\frac{z}{1024}\right)^{1024}$, range: $[-2299,251] \times[-1700,1700]$ 


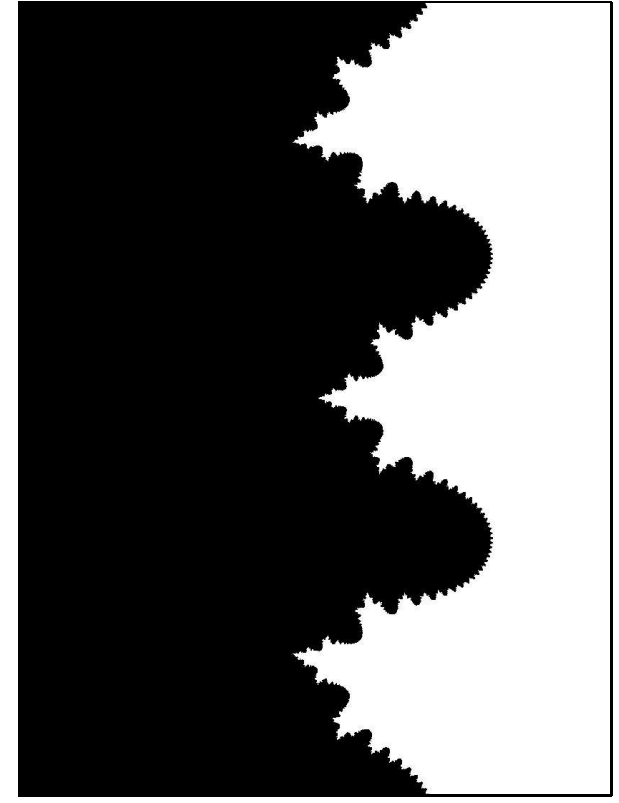

Figure 3.15: Filled Julia set of $P_{32}(z)=\left(1-\frac{1}{32}\right) z-1+\left(1+\frac{z}{32}\right)^{32}$, range: $[-7.5,7.5] \times[-10,10]$

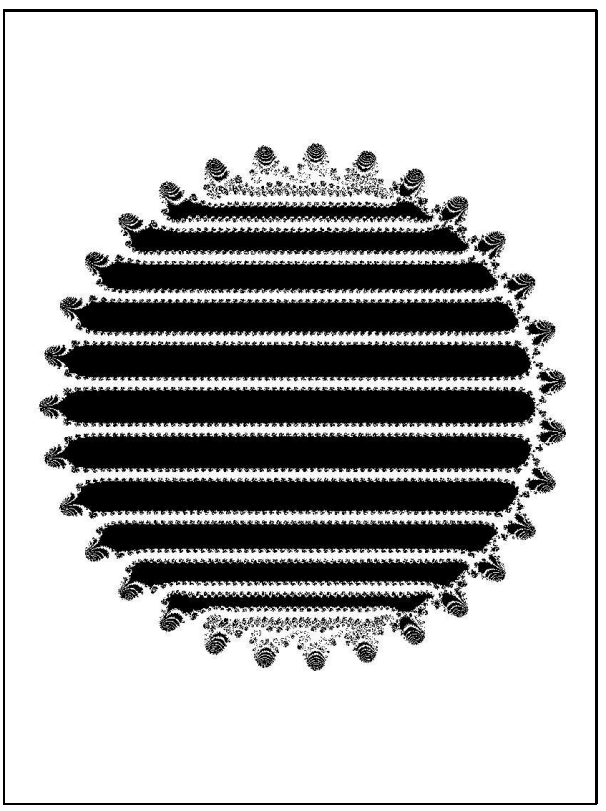

Figure 3.17: Filled Julia set of $Q_{32}(z)=z-1+\left(1+\frac{1}{32}\right)^{32}$, range: $[-71.75,-7.75] \times[-55,55]$

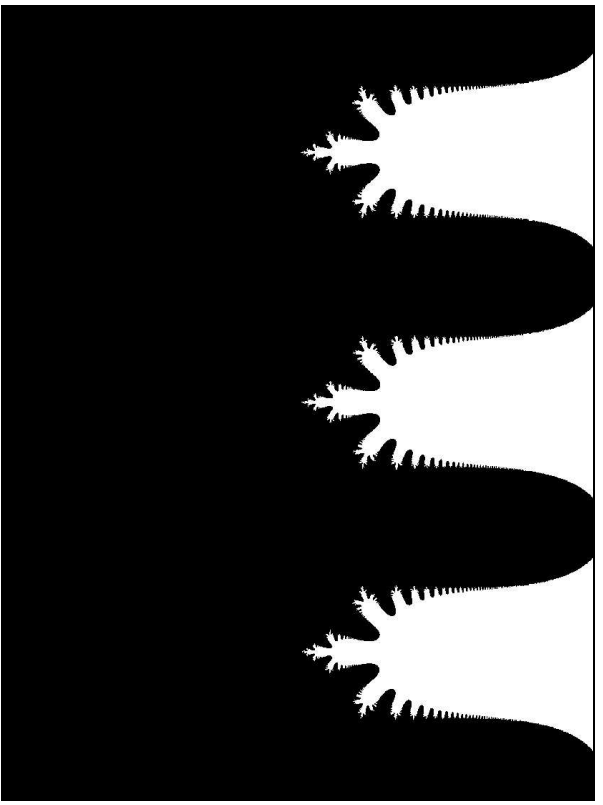

Figure 3.16: Filled Julia set of $P_{1024}(z)=\frac{1023}{1024} z-1+\left(1+\frac{z}{1024}\right)^{1024}$, range: $[-7.5,7.5] \times[-10,10]$

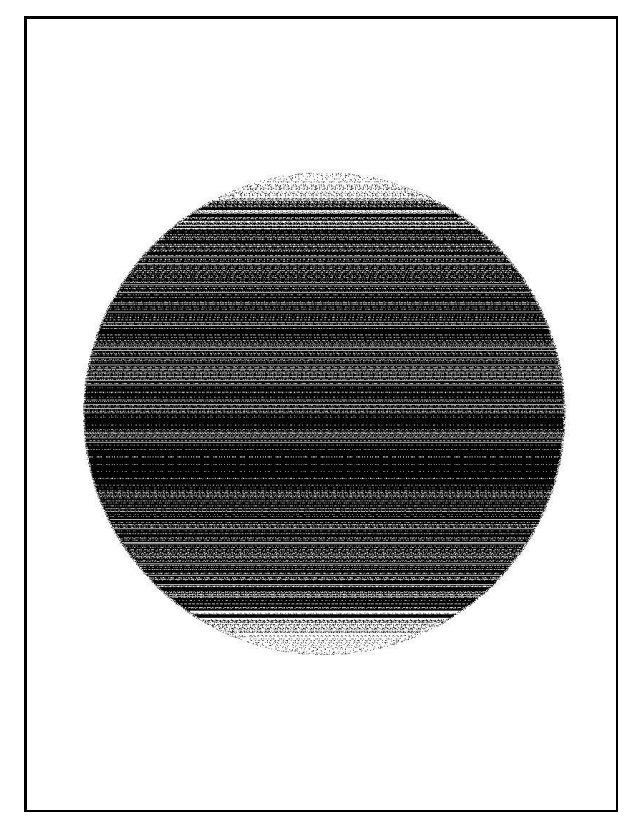

Figure 3.18: Filled Julia set of $Q_{1024}(z)=z-1+\left(1+\frac{1}{1024}\right)^{1024}$, range: $[-2299,251] \times[-1700,1700]$ 


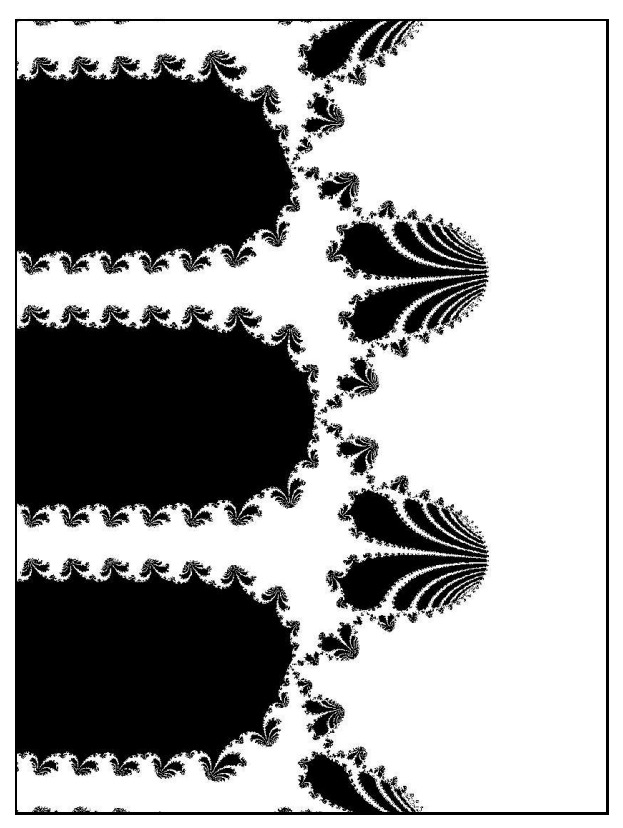

Figure 3.19: Filled Julia set of $Q_{32}(z)=z-1+\left(1+\frac{z}{32}\right)^{32}$, range: $[-7.5,7.5] \times[-10,10]$

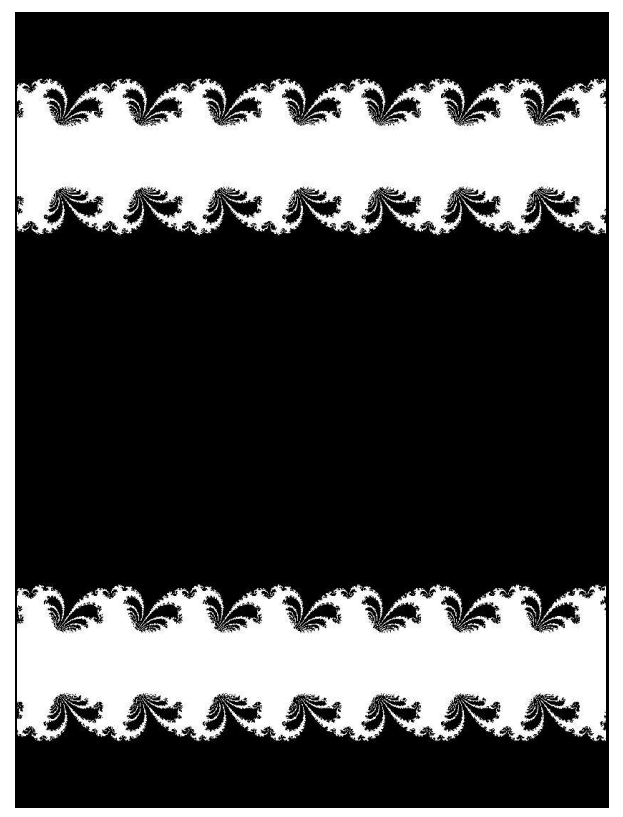

Figure 3.21: Filled Julia set of $Q_{32}(z)=z-1+\left(1+\frac{z}{32}\right)^{32}$, range: $[-35.75,-28.25] \times[-5,5]$

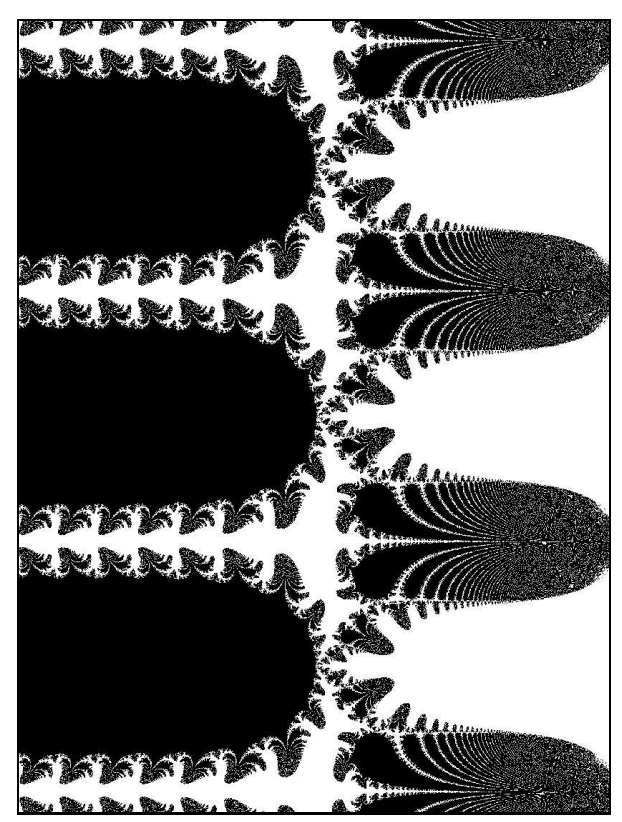

Figure 3.20: Filled Julia set of $Q_{1024}(z)=z-1+\left(1+\frac{z}{1024}\right)^{1024}$, range: $[-7.5,7.5] \times[-10,10]$

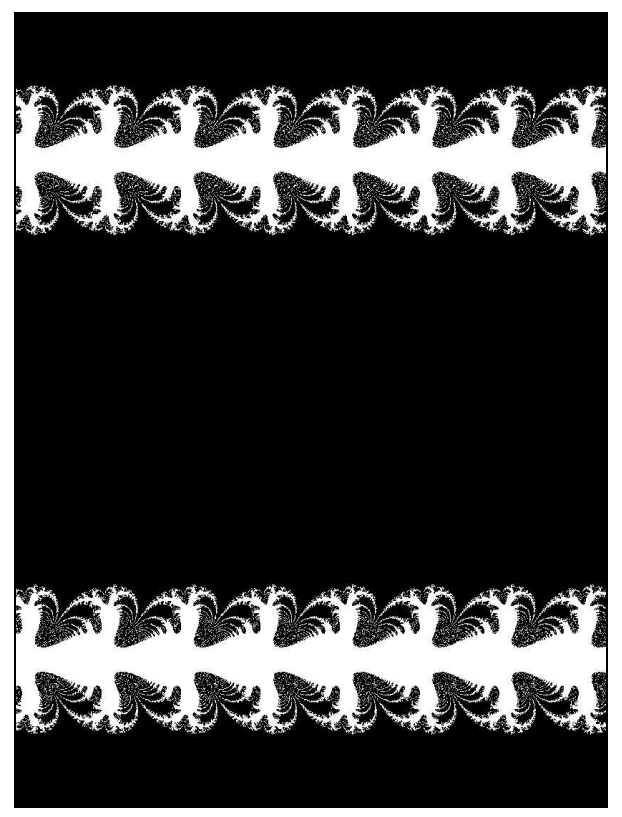

Figure 3.22: Filled Julia set of $Q_{1024}(z)=z-1+\left(1+\frac{z}{1024}\right)^{1024}$, range: $[-35.75,-28.25] \times[-5,5]$ 


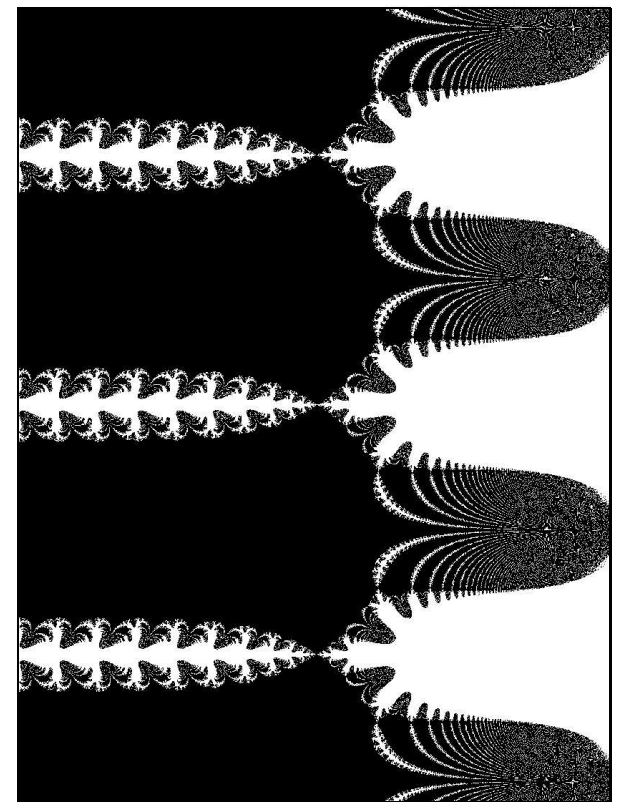

Figure 3.23: Filled Julia set of $Q_{1025}(z)=z-1+\left(1+\frac{z}{1025}\right)^{1025}$, range: $[-7.5,7.5] \times[-10,10]$

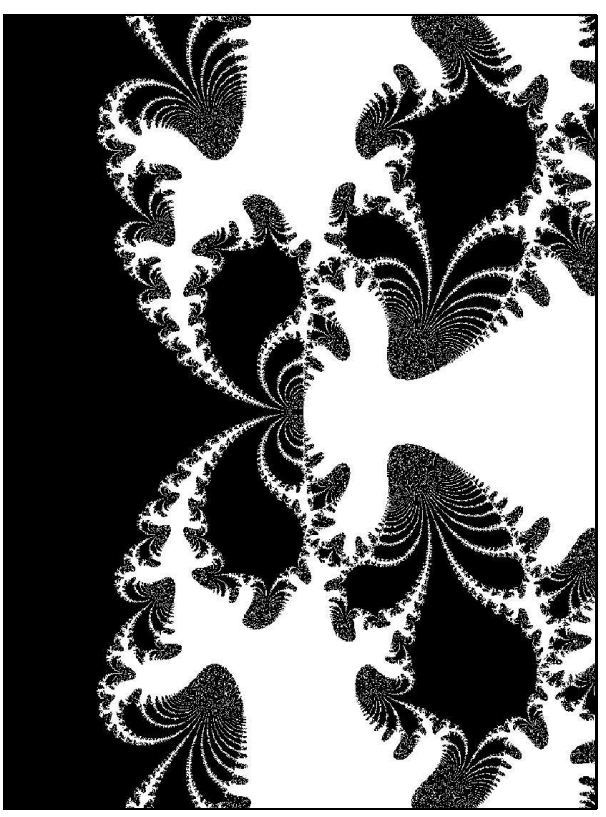

Figure 3.25: Filled Julia set of

$Q_{1024}(z)=z-1+\left(1+\frac{z}{1024}\right)^{1024}$ conjugated with $z \mapsto \frac{1}{z}$,

range: $[-0.375,0.375] \times[-0.5,0.5]$

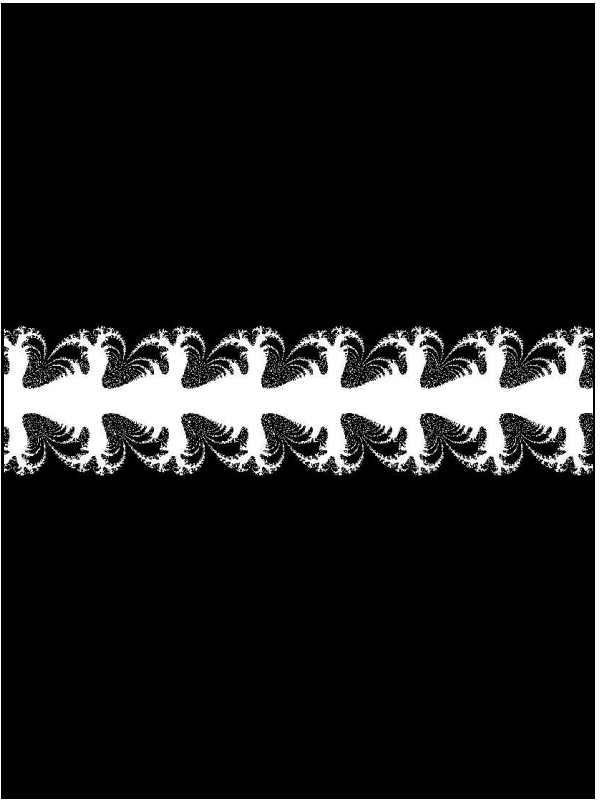

Figure 3.24: Filled Julia set of $Q_{1025}(z)=z-1+\left(1+\frac{z}{1025}\right)^{1025}$, range: $[-35.75,-28.25] \times[-5,5]$

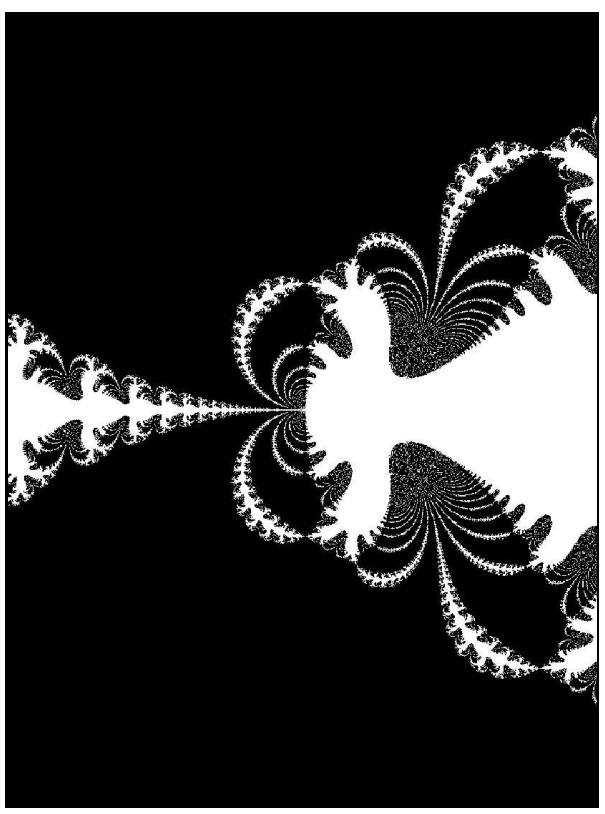

Figure 3.26: Filled Julia set of

$Q_{1025}(z)=z-1+\left(1+\frac{z}{1025}\right)^{1025}$ conjugated with $z \mapsto \frac{1}{z}$,

range: $[-0.375,0.3755] \times[-0.5,0.5]$ 
We already know that we can find a domain $U$ containing 0 such that $f_{1}$ is polynomial-like of degree 2 on $U$. Since this property is stable under perturbation, we find domains $U_{n}$ containing 0 such that $P_{n}$ is polynomial-like of degree 2 on $U_{n}$. Using the straightening theorem we see that each $P_{n}$ is hybrid equivalent to a quadratic polynomial $Q_{c_{n}}$, where $Q_{c_{n}}=z^{2}+c_{n}, c_{n} \in\left(-\frac{3}{4}, \frac{1}{4}\right)$ and $c_{n} \rightarrow \frac{1}{4}$ as $n$ tends to $\infty$. $f_{1 \mid U}$ is hybrid equivalent to $Q_{\frac{1}{4}}$. In various papers it has been found that $J\left(Q_{c_{n}}\right) \rightarrow J\left(Q_{\frac{1}{4}}\right)$ in the Hausdorff metric, so $J\left(P_{n}\right) \rightarrow J\left(f_{1}\right)$.

Another way to come to the same conclusion is to use McMullen's theorems in section 4.4.

For Siegel disks we can do even better in this family: since the filled Julia sets of quadratic polynomials having Siegel disks move continuously, also the closure of the attractive basin of $\infty$ of these polynomials moves continuously. Using the straightening map we can transfer this result to the family $\mathcal{F}_{a}$, yielding that the Baker domains move continuously even if there are Siegel disks.

\subsection{Bifurcations of Baker domains}

In this section we will investigate the limit processes

$f_{c}(z)=f_{c_{1}+i c_{2}}(z)=z-c_{1}-i c_{2}+e^{z} \rightarrow f_{i c_{2}}$ and study the limit functions. In particular, we are interested in what happens to the Baker domains and if these changes are "smooth". This will give us Baker domains of a different case of König's classification than before and examples of instable Baker domains and wandering domains. We will see that if we approach these limit functions with polynomials we will obtain similar results as in the preceding section.

\subsubsection{The dynamical plane}

Our main tool will be the fact that each $f_{c}$ is a logarithmic lift of an entire transcendental function, an easy calculation shows that if $g_{\lambda}(z)=\lambda z e^{z}$ and $\lambda=e^{-c}$, then $\exp \circ f_{c}=g_{\lambda} \circ \exp$.

The family $\left\{g_{\lambda}(z)=\lambda z e^{z}, \lambda \in \mathbb{C}^{*}\right\}$ has been intensively studied, for example in [20], [21] and [27], while the case $f_{c}, c \in \mathbb{R}^{+}$, is examined in [43].

Before we state a theorem, recall the definition of the Brjuno set: 
Definition 3 (Brjuno set)

Let $\theta \in \mathbb{R} \backslash \mathbb{Q}$ and $\left(\frac{p_{n}}{q_{n}}\right)_{n \in \mathbb{N}}$ be the rational approximations to $\theta$ coming from the continued fraction expansion. The set of Brjuno numbers is defined as

$$
\mathcal{B}=\left\{\theta \in \mathbb{R} \backslash \mathbb{Q}: \sum_{n \in \mathbb{N}} \frac{\log q_{n+1}}{q_{n}}<\infty\right\}
$$

Our main result in this section is the

\section{Theorem 10}

Let $f_{c}(z)=z-c+e^{z}, c \in i \mathbb{R}$. We have the following possibilities:

a)

If $c=0$, then the Fatou set of $f_{c}$ consists of infinitely many Baker domains and their preimages.

b)

If $c=2 \pi i p / q, p / q \in \mathbb{Q} \backslash\{0\},(p, q)=1$, then the Fatou set of $f_{c}$ consists of wandering domains only. Let $k$ be the smallest integer larger than $|p / q|$, then the number of asymptotic paths of wandering domains is equal to $k$ times the number of parabolic petals of $g_{\exp (-c)}$ at 0 .

c)

If $c=2 \pi i \alpha$, where $\alpha$ is contained in the Brjuno set, then the Fatou set of $f_{c}$ consists of a univalent Baker domain and its preimages.

d)

If $c=2 \pi i \alpha$, where $\alpha$ is irrational and not contained in the Brjuno set, then the Fatou set of $f_{c}$ is empty.

Remark 13 All results we will use for the case $\lambda \in \mathbb{D}^{*}$ can be found in section 3.2; actually, it is easy to see a little bit more: it has been proved that $g_{\lambda}$ is structurally stable if $\lambda \in \mathbb{D}^{*}$ ([31]), so for $\lambda_{1}, \lambda_{2} \in \mathbb{D}^{*}$ the functions $g_{\lambda_{1}}, g_{\lambda_{2}}$ are quasiconformally conjugate. Using the same ideas as in [14] we can see that the Baker domain is in fact the only Fatou component of $f_{-\ln \lambda_{0}}$ for some $\lambda_{0} \in \mathbb{D}^{*}$ and therefore for all $\lambda \in \mathbb{D}^{*}$, so these Baker domains are completely invariant as are the coresponding attracting basins of $g_{\lambda}$.

In section 4.4 we will give details on the location of the singular values of $f_{c}$, but since we do not need that in the following chapters, we did not include this in our theorem.

We note that the Baker domain can vanish or split into infinitely many components; König remarked that in the cases a) and c) the Baker domains belong to the second class of his classification, so in any case the functions 
are dynamically distinguishable from the functions we dealt with in section 2.3 .

\subsubsection{Radial continuity of Julia sets}

If we have a holomorphically parameterized family of rational maps and a sequence of parameters contained in a hyperbolic component approaches a point on the boundary "nicely" (that is, not tangential to the boundary), then the corresponding Julia sets converge to the Julia set of the limit function (see [36]). For entire transcendental functions this result is not known, but at least in our special case the analogous result holds:

\section{Theorem 11}

For $\lambda \in \partial \mathbb{D}$ and $\varepsilon>0, J\left(g_{(1-\varepsilon) \lambda}\right)$ converges to $J\left(g_{\lambda}\right)$ as $\varepsilon$ tends to 0 .

Using the lift, we obtain the corresponding result for our family $\left\{f_{c}\right\}$ :

Corollary 4

Let $c \in \mathbb{R}$ and $\varepsilon>0$. Then $J\left(f_{\varepsilon+i c}\right)$ converges to $J\left(f_{i c}\right)$ as $\varepsilon$ tends to 0 .

\section{Remark 14}

Morosawa and Taniguchi [43] proved this result for $c=0$ directly in the c-plane, but it seems to be difficult to carry over their method to all $c \in \mathbb{R}$. 


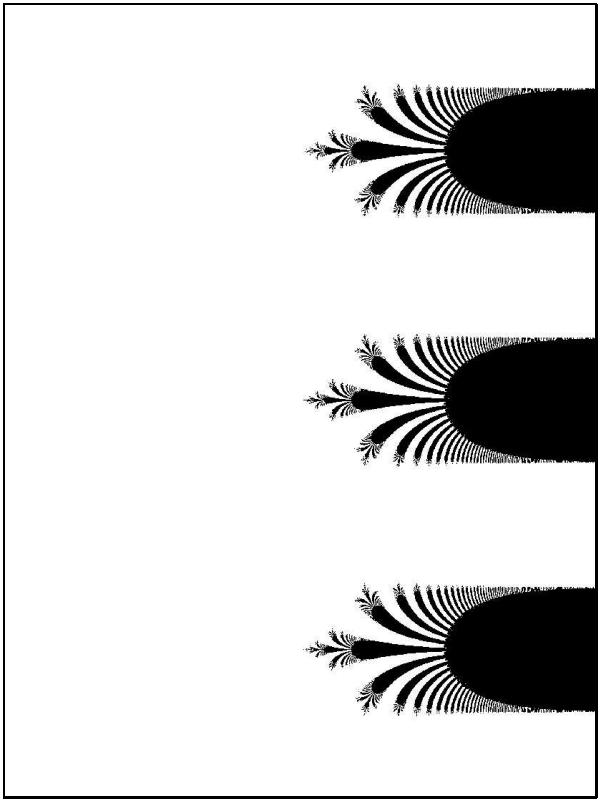

Figure 3.27: Julia set of $f(z)=z-1+e^{z}$, range: $[-7.5,7.5] \times[-10,10]$

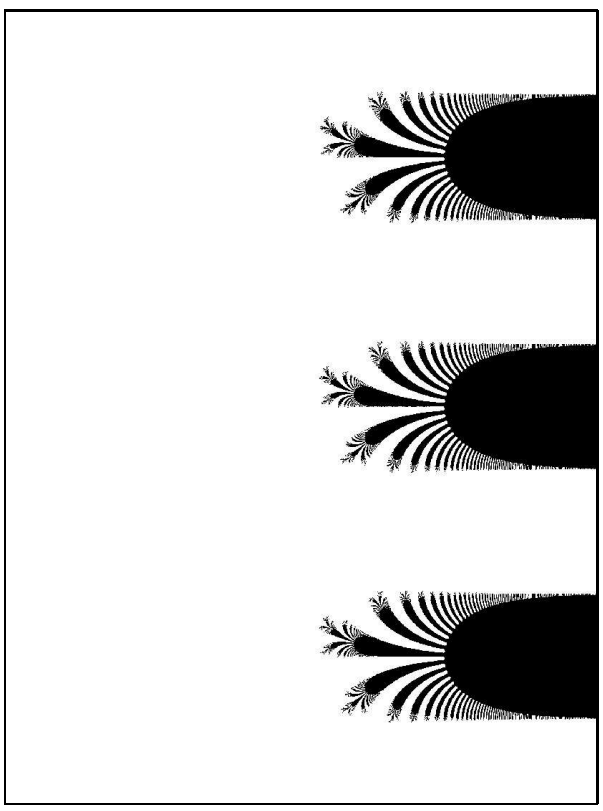

Figure 3.29: Julia set of $f(z)=z-1+i \frac{\pi}{2}+e^{z}$, range: $[-7.5,7.5] \times[-10,10]$

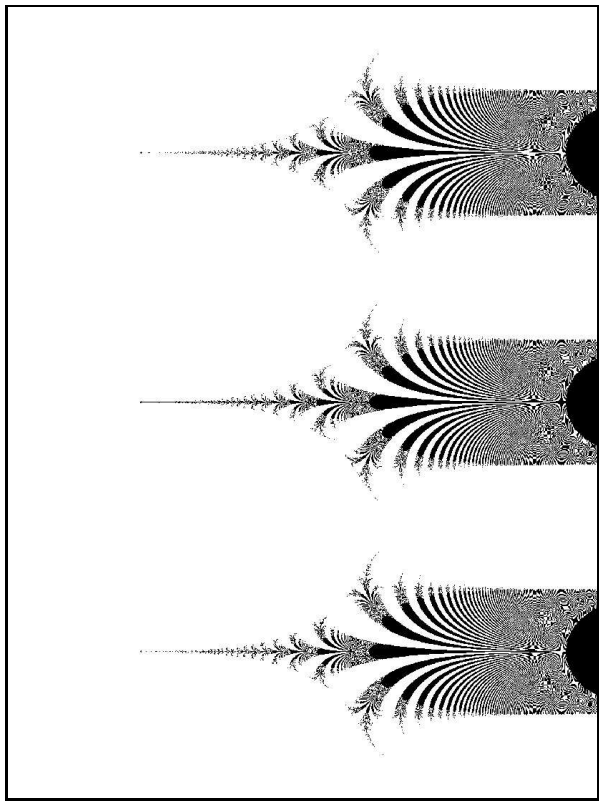

Figure 3.28: Julia set of $f(z)=z-0.000001+e^{z}$, range: $[-7.5,7.5] \times[-10,10]$

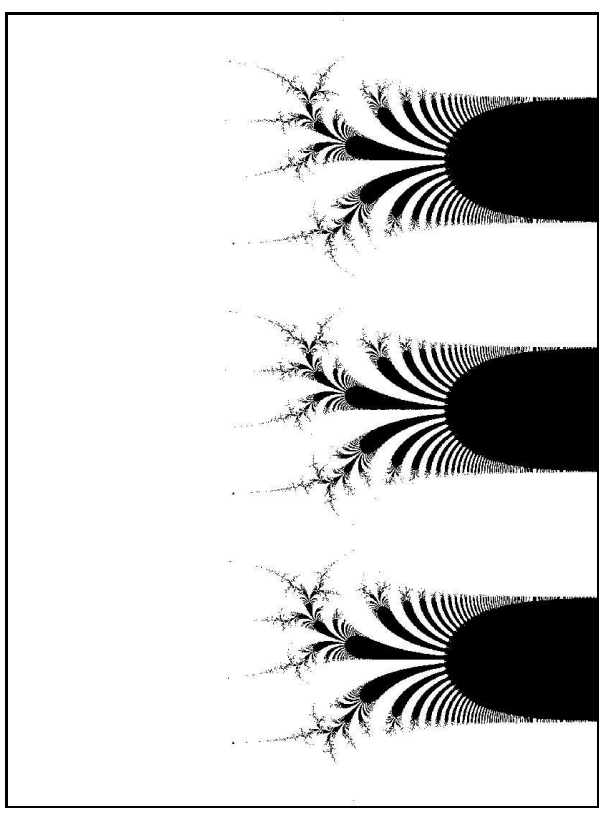

Figure 3.30: Julia set of $f(z)=z-0.01+i \frac{\pi}{2}+e^{z}$, range: $[-7.5,7.5] \times[-10,10]$ 


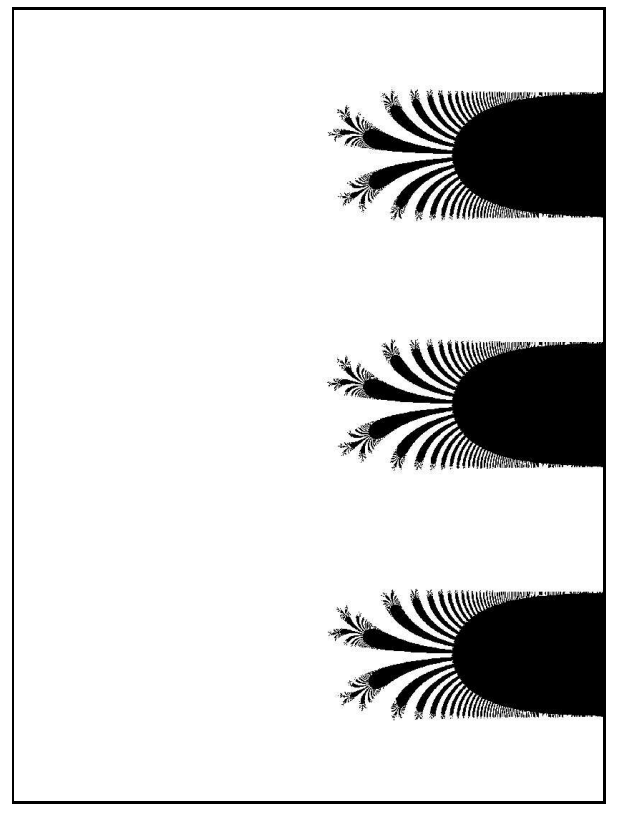

Figure 3.31: Julia set of $f(z)=z-1+i(1+\sqrt{5}) \pi+e^{z}$, range: $[-7.5,7.5] \times[-10,10]$

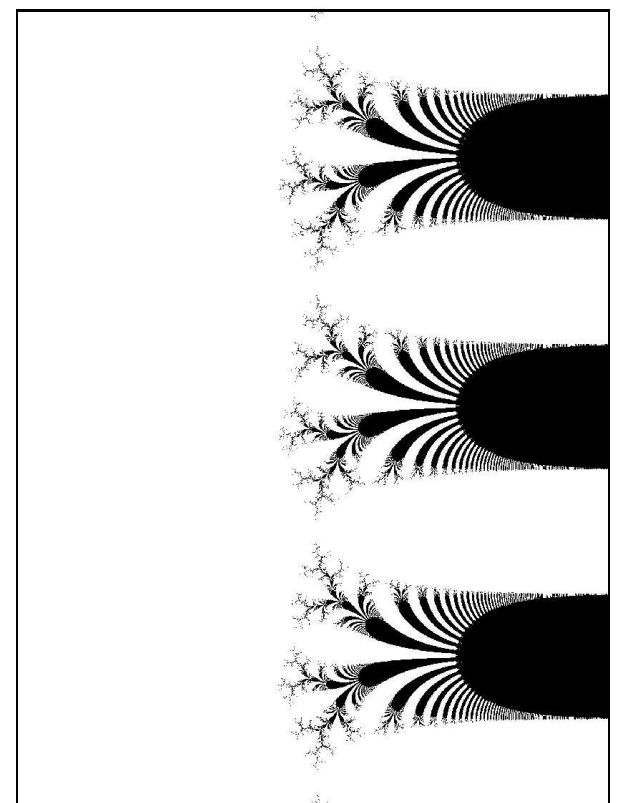

Figure 3.32: Julia set of $f(z)=z-0.01+i(1+\sqrt{5}) \pi+e^{z}$, range: $[-7.5,7.5] \times[-10,10]$ 


\subsubsection{Approximation of Baker domains and wandering domains}

Again, we can approximate the functions $f(z)=z-c+e^{z}, c \in i \mathbb{R}$, with polynomials and ask if the Julia sets of the polynomials converge to the Julia sets of the limit functions. The following theorem shows that the results are similar to the results in section 3.3 .

\section{Theorem 12}

Let $f(z)=z-c+e^{z}, c \in i \mathbb{R}$. Then there exists a sequence of entire transcendental functions $f_{n}$ and sequences $P_{n}$ and $Q_{n}$ of polynomials such that $f_{n} \rightarrow f, P_{n} \rightarrow f$ and $Q_{n} \rightarrow f$ and

a)

b)

$J\left(f_{n}\right)=\overline{\mathbb{C}}$ for all $n \in \mathbb{N}$

c)

$J\left(P_{n}\right) \rightarrow J(f)$ in the Hausdorff metric

If $\frac{c}{2 \pi i} \in \mathbb{Q} \cup \mathcal{B}$, then $J\left(Q_{n}\right) \nrightarrow J(f)$ in the Hausdorff metric. In particular, we can take the polynomials $Q_{n}$ such that $J\left(Q_{n}\right) \rightarrow \overline{\mathbb{C}} \neq J(f)$. If $\frac{c}{2 \pi i} \notin \mathbb{Q} \cup \mathcal{B}$, then such a sequence of entire functions $g_{n}$ with $g_{n} \rightarrow f, J\left(g_{n}\right) \nrightarrow J(f)$ in the Hausdorff metric does not exist.

Of course, the case $\frac{c}{2 \pi i} \in \mathbb{Q}$ provides a direct link between the parabolic implosion and the instability of Baker domains. 


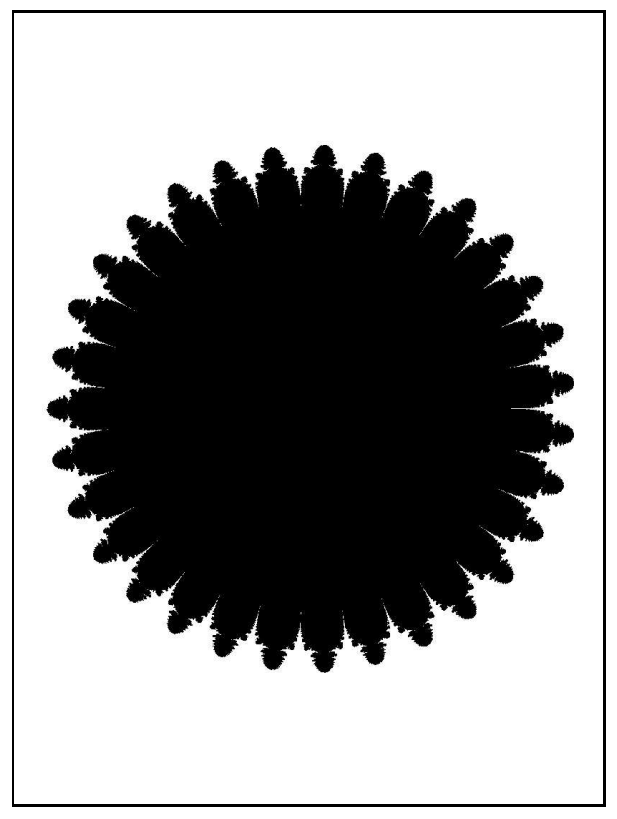

Figure 3.33: Filled Julia set of $f(z)=z-0.01+\left(1+\frac{z}{32}\right)^{32}$, range: $[-71.75,-7.75] \times[-55,55]$

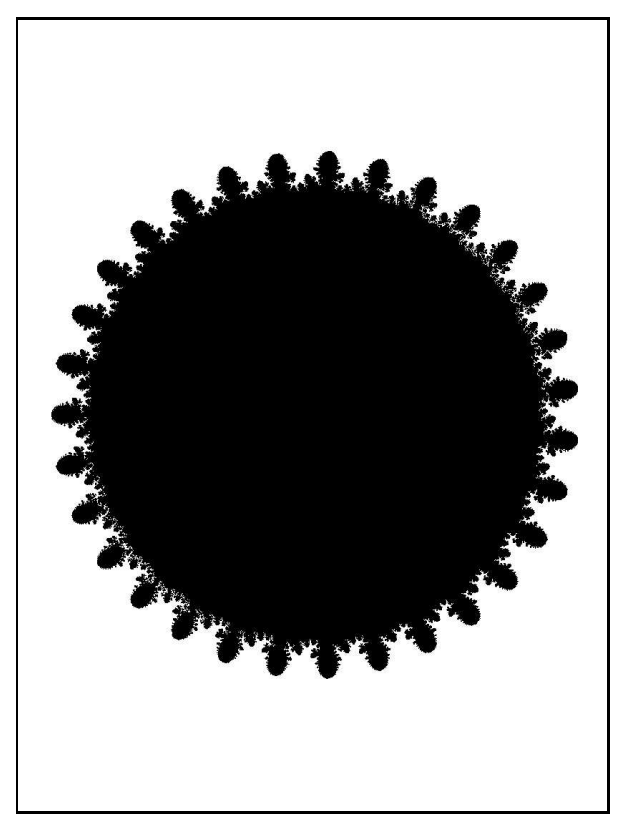

Figure 3.35: Filled Julia set of

$f(z)=z-0.01+i \frac{\pi}{2}+\left(1+\frac{z}{32}\right)^{32}$, range: $[-71.75,-7.75] \times[-55,55]$

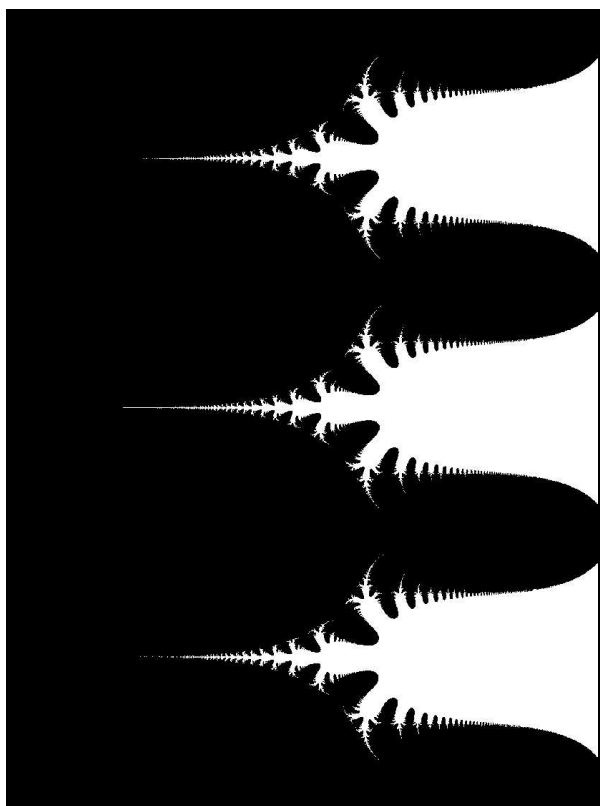

Figure 3.34: Filled Julia set of $f(z)=z-0.01+\left(1+\frac{z}{1024}\right)^{1024}$, range: $[-7.5,7.5] \times[-10,10]$

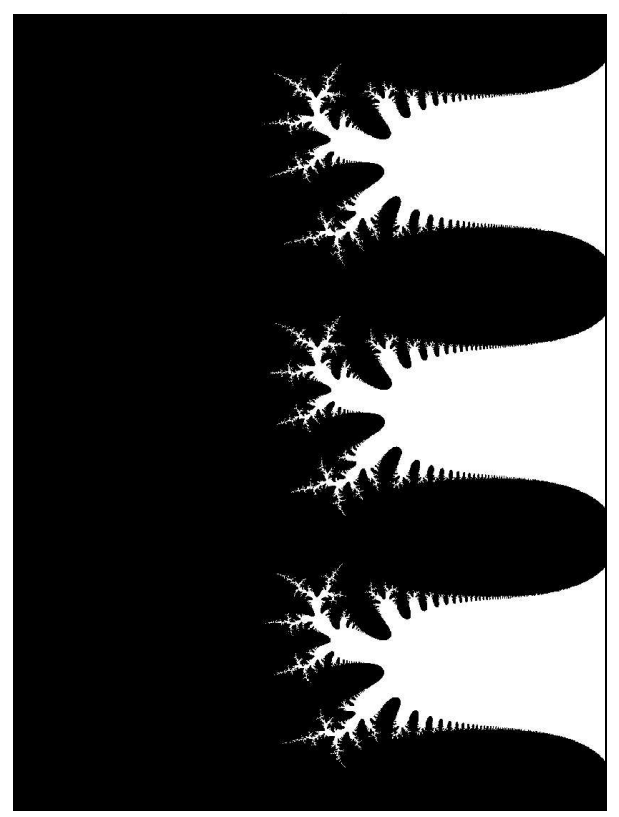

Figure 3.36: Filled Julia set of $f(z)=z-0.01+i \frac{\pi}{2}+\left(1+\frac{z}{1024}\right)^{1024}$, range: $[-7.5,7.5] \times[-10,10]$ 


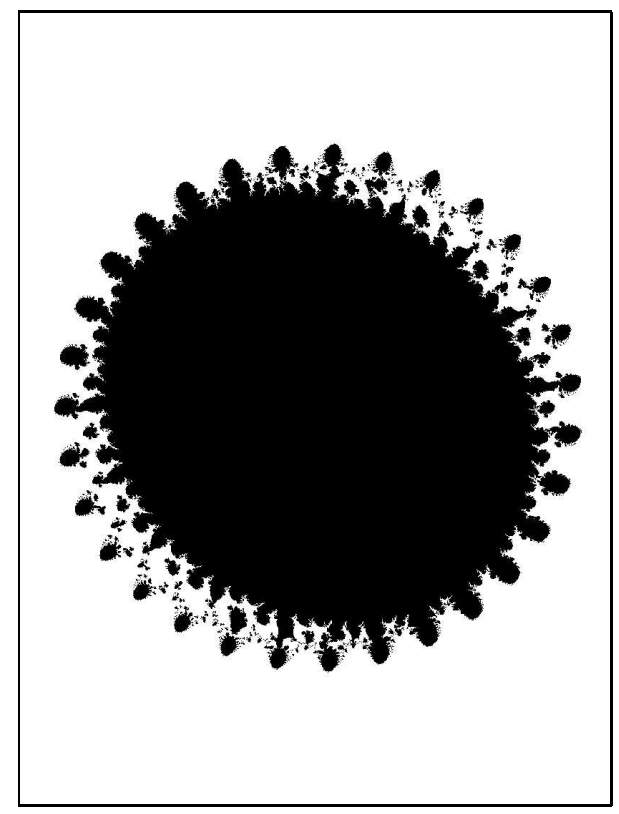

Figure 3.37: Filled Julia set of $f(z)=z-0.01+i(1+\sqrt{5}) \pi+(1+$ $\left.\frac{z}{32}\right)^{32}$,

range: $[-71.75,-7.75] \times[-55,55]$

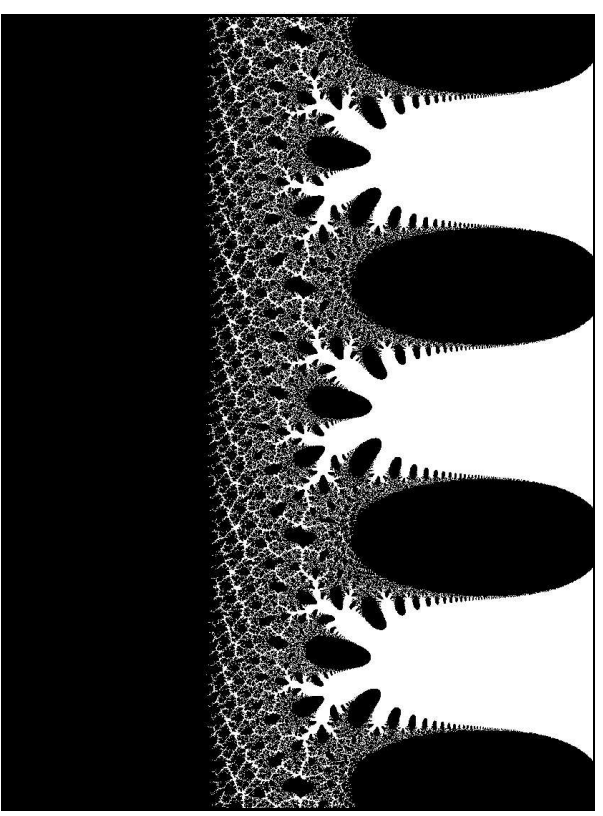

Figure 3.38: Filled Julia set of $f(z)=z-0.01+i(1+\sqrt{5}) \pi+(1+$ $\left.\frac{z}{1024}\right)^{1024}$,

range: $[-7.5,7.5] \times[-10,10]$ 


\subsubsection{Stability}

König raised the question which of his classes of Baker domains are stable under perturbations. Using our previous results we may answer this question for our one-parameter-families.

In the following, a "Baker domain of type 1" is a Baker domain belonging to the first class of König's theorem, etc..

In 3.3 we have seen that Baker domains of type 1 can be stable. Our results in 3.4.1 give that Baker domains of type 2 may be unstable. To make this statement more precise, we formulate the

\section{Lemma 12}

Let $f_{c}(z)=z-c+e^{z}, c \in \mathbb{C}$.

$f_{c}$ has a Baker domain if and only if one of the following holds:

a)

$\operatorname{Re} c>0$ or

b)

$c=0$ or

c)

$\frac{c}{2 \pi i} \in \mathcal{B}$.

In the first case and second case the Baker domains of $f_{c}$ are of type 1 , in the third case all Baker domains are of type 2.

Hence, the set $\left\{c: f_{c}\right.$ has a Baker domain of type 1$\}$ is the right half plane plus the point 0 ; the set $\left\{c: f_{c}\right.$ has a Baker domain of type 2$\}$ is a Cantor set, in particular, it has empty interior.

On the other hand, we can find a holomorphically parameterized family such that a function $f_{c}$, where $\operatorname{Re} c>0$, is not stable in this family; recall that by Bergweiler's estimations on Baker domains for any invariant Baker domain $B$ of an entire transcendental function $f$ we can find a forward invariant curve $\gamma \subset B$ such that $|f(z)|<A|z|$ for some $A>0$. Hence, $\gamma$ is an asymptotic path of the asymptotic value 0 of the meromorphic function

$g: \mathbb{C} \rightarrow \overline{\mathbb{C}} ; z \mapsto \frac{f(z)}{z^{2}}$. Now consider for a fixed $c$ contained in the right half plane the functions $f_{\lambda, c}(z)=\lambda z^{2}+z-c+e^{z}$. Clearly, $f_{\lambda, c} \rightarrow f_{c}$ as $\lambda \rightarrow 0$. Since 0 is the only asymptotic value of the function $z \mapsto \frac{e^{z}}{z^{2}}, g$ has only one asymptotic value, namely $\lambda$. We deduce that $f_{\lambda, c}$ has no invariant Baker domain if $\lambda \neq 0$, so the set $\left\{\lambda: f_{\lambda, c}\right.$ has an invariant Baker domain of type 1$\}$ has empty interior. But this case might be an example of a singularity in the parameter plane as in 3.1.3, since it is easy to check that the set of critical points does not move continuously with respect to the Euclidean metric in 0 .

The result of Rippon and Stallard mentioned in the introduction gives that 
there exists holomorphically parameterized families such that all of these functions have Baker domains of type 3, for example, all of the functions $f_{\lambda}(z)=2 z+\lambda e^{z}, \lambda \in \mathbb{C}^{*}$, have such a Baker domain. Using the same argument as above we see that the set

$\left\{\lambda: g_{\lambda, c}\right.$ has an invariant Baker domain of type 3$\}=\{0\}$ if we define $g_{\lambda}$ by $g_{\lambda}(z)=\lambda z^{2}+2 z+e^{z}$.

In an analogous manner one can do this for Baker domains of type 2 as well. So, functions having Baker domains can be imbedded in families making them stable or unstable, comparably to functions having parabolic cycles or Siegel disks. None of these types is always stable as are attracting basins. Nevertheless, by restricting the space of functions we may try to obtain stronger results:

Let $g$ be an entire transcendental function of bounded type such that $g(0)=0$ and $O^{-}(0)=\{0\}$. Then $g(z)=\lambda z^{m} e^{h(z)}$, where $\lambda \in \mathbb{C}^{*}, m \in \mathbb{N}$ and $h$ is entire. By Bergweiler's result in [8] there exists a logarithmic lift $f$ of $g$. Using the linearizations of Fatou components of $g$ one can see that $f$ has a Baker domain of type 3 if and only if $g$ has a super-attracting fixed point at 0 (this happens if and only if $m>1$ ), $f$ has a Baker domain of type 1 if and only if $g$ has a parabolic fixed point at 0 with only one petal attached to 0 or an attracting but not super-attracting fixed point at 0 , and $f$ has a Baker domain of the second type iff 0 is the center of a Siegel disk. If we perturb $g$ we see how the Baker domain of $f$ changes (or does not change). A closer look reveals that for any family $\mathcal{F}=\left\{f_{\lambda}\right\}_{\lambda \in \Lambda}$ holomorphically parameterized by a complex manifold $\Lambda$ of such functions the set $M_{2}=\{f \in \mathcal{F}: f$ has a Baker domain of type 2$\}$ is closed, possibly with empty interior, and $M_{3}=\{f \in \mathcal{F}: f$ has a Baker domain of type 3$\}$ is empty or equal to $\Lambda$. The analogy to more explored Fatou components in this case motivates questions if one can enlarge the class of "allowed" functions as the following one:

\section{Question 1}

Can one give some conditions such that for any family $\mathcal{F}=\left\{f_{\lambda}\right\}_{\lambda \in \Lambda}$ of entire transcendental functions having Baker domains and satisfying these conditions the set $\{f \in \mathcal{F}: f$ has a Baker domain of type 2$\}$ is closed and $\{f \in \mathcal{F}: f$ has a Baker domain of type 3$\}$ is open?

We conjecture that the functions $f_{\lambda}(z)=\lambda z-1-e^{z}$ have Baker domains of type 3 if and only if $\lambda \in(1, \infty)$ and a Baker domain of type 1 only for $\lambda=1$. If this is true, then the condition " $\mathcal{F}$ is holomorphically parameterized and the set of critical points moves continuously with respect to the Euclidean metric with the parameter" is not sufficient. Hence, these conditions have to 
be quite restrictive (as the condition that all $f_{\lambda}$ are logarithmic lifts). König proposed to use methods similar to the $\lambda$-Lemma for rational functions to work on this problem.

\subsection{Open questions}

As mentioned before, the question of the structure of the possible limit sets of sequences of polynomials tending to an entire transcendental function having Baker domains is still open; we have seen that $\overline{\mathbb{C}}$ is a possible limit set, but one may ask if it the only limit set not having empty interior. Note that the analogous question concerning the parabolic implosion for entire transcendental functions is also still open.

A related problem is formulated in Conjecture 1.

Next, we suppose that the calculations in 3.2 and 3.3 can be generalized, for example one may try to take $P$ to be a entire transcendental or rational function. This might help to better understand Baker domains of meromorphic functions.

Another question is formulated in section 3.4.4.

Finally, there are more questions concerning the approximation of entire transcendental functions by polynomials. We only want to mention the following: $f_{c}(z)=z+c-e^{z}$ has wandering domains for $c=1+2 \pi i$ (it is similar to Herman's example). For all sequences $g_{n}$ of entire functions tending to $f$ we have $\lim _{n \rightarrow \infty} J\left(g_{n}\right)=J\left(f_{1}\right)$, since the Fatou set of $f_{1}$ consists of basins of super-attracting fixed points only. Furthermore, $J\left(f_{1}\right)=J\left(f_{1+2 \pi i}\right)$. Do we have $\lim _{n \rightarrow \infty} J\left(h_{n}\right)=J\left(f_{1+2 \pi i}\right)$ for all sequences $h_{n}$ of entire functions tending to $f_{1+2 \pi i}$ ? 


\section{Chapter 4}

\section{Proofs}

\subsection{A one-parameter family}

\subsubsection{The dynamical plane}

\subsubsection{Existence of a Baker domain}

Let $\delta \in(0,1)$ and

$$
\begin{aligned}
M_{\delta} & =\left\{z:\left|g_{b}(z)-(z-1)\right|<\delta\right\} \\
& =\left\{x+i y: y^{2}<\delta^{2} \frac{e^{-2 x}}{|b|^{2}}-x^{2}\right\} .
\end{aligned}
$$

We will use later that a point $z$ in $M_{\delta}$ satisfies $g_{b}(z) \in D(z-1, \delta)$, in particular, $\operatorname{Re} g_{b}(z)<\operatorname{Re} z-(1-\delta)$. Some short calculations give

\section{Lemma 13}

For any $c>0$, the function $x \mapsto c \frac{e^{-2 x}}{|b|^{2}}-x^{2}$ has at most one maximum and one minimum. Both the maximum value and minimum value are smaller than $1 / 4$, while the maximum and minimum are in $\mathbb{R}_{0}^{-}$.

\section{Proof:}

Let $h(x)=c \frac{e^{-2 x}}{|b|^{2}}-x^{2}$. Then $h^{\prime}(x)=-2\left(c \frac{e^{-2 x}}{|b|^{2}}+x\right)$. Any critical point $x$ of $h$ satisfies $c \frac{e^{-2 x}}{|b|^{2}}=-x$, so $h(x)=-x-x^{2} \leq 1 / 4$. Furthermore, $h^{\prime \prime}(x)=4 c \frac{e^{-2 x}}{|b|^{2}}-2$ has at most one root, so there are at most two roots of $h^{\prime}$. Finally, $h^{\prime}(x)<0$ on $\mathbb{R}^{+}$. 
Noting that $h(z) \rightarrow+\infty$ as $x \rightarrow-\infty$, we obtain the

\section{Corollary 5}

$M_{\delta}$ is non-empty, symmetric with respect to the real axis, consists of at most two components and exactly one component is unbounded. If there are two components, then the bounded one is contained in the strip $\{z:|\operatorname{Im} z| \leq 1 / 2\}$. If $z \in M_{\delta}$ and $\operatorname{Im} z>1 / 2$, then $x+i y \in M_{\delta}$ for all $x, y$ satisfying $x<R e z,|y|<\operatorname{Im} z$. Furthermore, if $x \in M_{\delta}^{C} \cap(0, \infty)$, then $M_{\delta} \subset\{z: \operatorname{Re} z<x\}$.

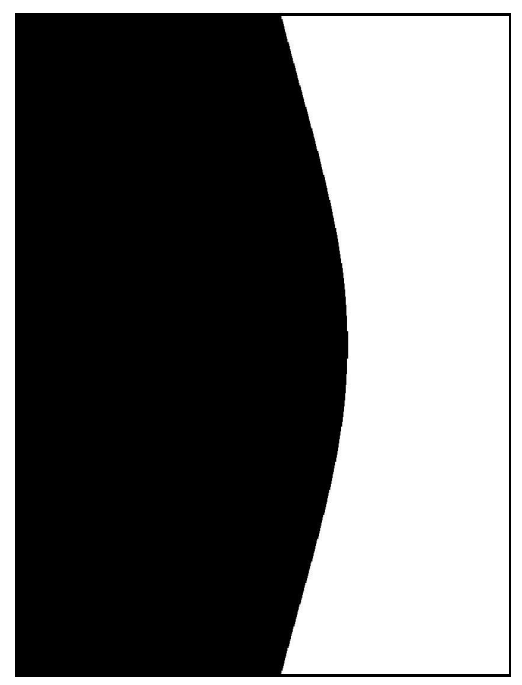

Figure 4.1: $M_{1}$ for $|b|=0.1$

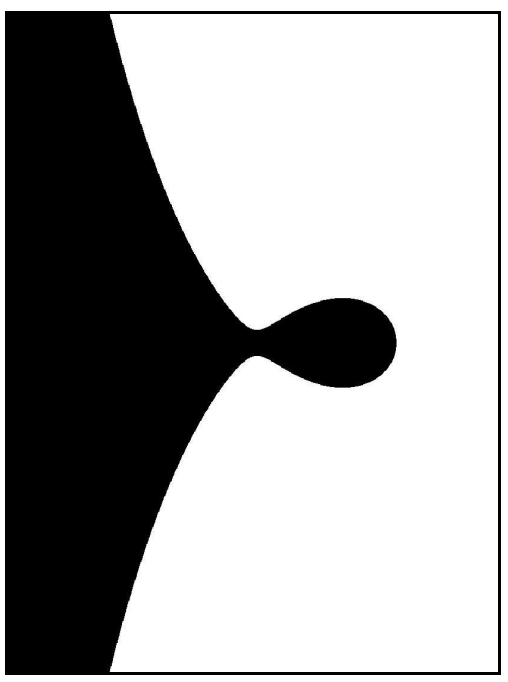

Figure 4.2: $M_{1}$ for $|b|=2.7$ 


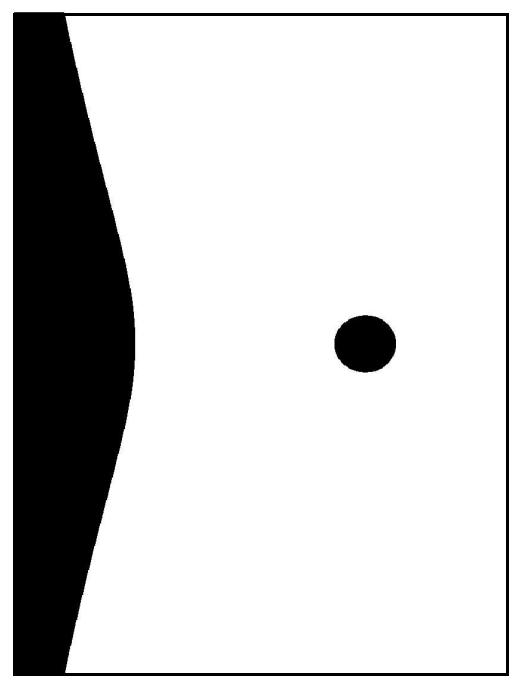

Figure 4.3: $M_{1}$ for $|b|=4$

Now we focus on the unbounded component. The functions $h_{1,2}(x)= \pm \delta\left(\frac{e^{-2 x}}{|b|^{2}}-x^{2}\right)^{1 / 2}$ are defined on an interval $(-\infty,-R)$, decrease/increase exponentially as $x$ tends to $-\infty$ and parameterize the boundary of $M_{\delta}$ on that half plane. Take $R=R(\delta)>0$ such that $\left|h_{1,2}^{\prime}(x)\right|>\frac{\delta}{1-\delta}$ on $(-\infty,-R)$. Then for all $z \in M_{\delta} \cap\{z: \operatorname{Re} z<-R\}=: D_{\delta, R}$ the disk $D(z-1, \delta)$ is contained in $D_{\delta, R}$. By definition of $M_{\delta}, D(z-1, \delta)$ contains $g_{b}(z)$, we conclude that $D_{\delta, R}$ is forward invariant with respect to $g_{b}$, even its boundary is mapped into itself. Since $\operatorname{Re} g_{b}(z)<\operatorname{Re} z-\delta$ for all $z \in M_{\delta}$, it is contained in a Baker domain $B_{b}$.

Note that this argumentation works for all $\delta \in(0,1)$ and also for any translation of a set $D_{\delta, R}$ to the left.

In the following, let $\delta$ and $R(\delta)$ be such that $D_{\delta, R}$ is forward invariant. Since $R(\delta)$ is fixed, we will drop an index and write $D_{\delta}$.

\section{Proposition 7}

For all $z \in B_{b}$ there exists an $n \in \mathbb{N}$ such that $g_{b}^{n}(z) \in D_{\delta}$.

\section{Proof:}

For any $z \in D_{\delta}$ we have $g_{b}(z) \in D(z-1, \delta)$, so $z_{n}=g_{b}^{n}(z)$ remains in the sector $\left\{x+i y: x<\operatorname{Re} z,\left|\frac{y-\operatorname{Im} z}{x-\operatorname{Re} z}\right|<\frac{\delta}{1-\delta}\right\}$. Hence, the Euklidean distance between the boundary of $D_{\delta}$ and $z_{n}$ tends to $\infty$ as $n$ tends to $\infty$.

The fixed points of $g_{b}$ are located on the boundary of the set $M_{1}=\left\{x+i y: y^{2}<\frac{e^{-2 x}}{|b|^{2}}-x^{2}\right\}=\left\{z:\left|b z e^{z}\right|=1\right\} . \partial M_{1}$ and $\partial D_{\delta}$ have an asymptotic distance (in the sense of Lemma 29) of $|\ln \delta|$. 


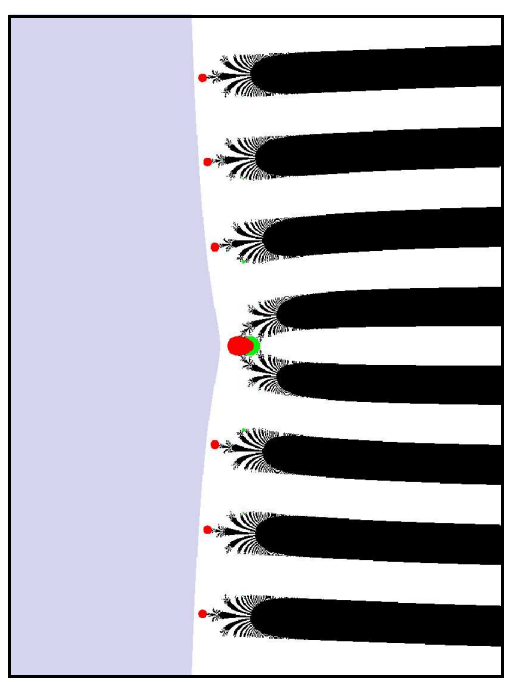

Figure 4.4: The Julia set of $g_{-3}$ is shown in black, the absorbing domain as constructed above (for $\delta=0.5$ and $R=1$ ) in blue and neighborhoods of the fixed points in red

Since $\left|\frac{d}{d z} b z e^{z}\right|=\left|b(z+1) e^{z}\right|=\left|b z e^{z}+b e^{z}\right| \approx\left|b z e^{z}\right|=1$ if $z \in \partial M_{1}$ and $|z|$ is sufficiently large, the fixed points have an asymptotic vertical distance of $2 \pi i$. As we will prove later, $g_{b}$ has at most one non-repelling cycle, so all fixed points except for at most one belong to the Julia set. Hence, we find a $K>0$ such that $d(z, \partial B)<|z-v|+K$ for all $z \in D_{\delta}$ and $v \in \partial D_{\delta}$. From the estimation $\frac{1}{2 d(z, \partial B)} \leq \rho(z) \leq \frac{2}{d(z, \partial B)}$ for the hyperbolic metric $\rho(z)|d z|$ on $B_{b}$ we obtain the following estimation for the hyperbolic distance on $B_{b}$ between $z_{n}$ and the boundary of $D_{\delta}$ :

Let $\gamma_{v, n}$ be the segment connecting $z_{n}$ and $v \in \mathbb{C}$.

$$
\begin{aligned}
{\left[z_{n}, \partial D_{\delta}\right]_{B_{b}} } & =\inf _{v \in D_{\delta}} \int_{\gamma_{v, n}} \frac{d z}{2 d(z, \partial B)} \\
& \geq \inf _{v \in \partial D_{\delta}} \frac{1}{2} \int_{\gamma_{v, n}} \frac{d z}{d(z, v)+K} \\
& =\inf _{v \in \partial D_{\delta}} \frac{1}{2} \int_{0}^{\left|v-z_{n}\right|} \frac{d x}{x+K} \\
& =\inf _{v \in \partial D_{\delta}} \frac{1}{2} \ln \left(\left|v-z_{n}\right|+K\right)-\ln K
\end{aligned}
$$

which tends to $\infty$ as $n$ tends to $\infty$. On the other hand, the hyperbolic met- 
ric is contracting, so for any $z^{\prime} \in B_{b}$ we have $\left[z_{n}, g_{b}^{n}\left(z^{\prime}\right)\right]_{B_{b}} \leq\left[z, z^{\prime}\right]_{B_{b}}$. This implies $z_{n}^{\prime} \in D_{\delta}$ for $n$ sufficiently large.

Hence, for any $\varepsilon>0$ and any $z \in B_{b}$ we find an $n_{0} \in \mathbb{N}$ such that

$g_{b}(z) \in\left\{z:\left|g_{b}(z)-(z-1)\right|<\varepsilon\right\}$ for all $n>n_{0}$. This proves the first statement of Proposition 1 .

\section{Corollary 6}

$D_{\delta}$ is an absorbing domain of $g_{b}$.

\section{Proof:}

The only condition we have to prove is the condition on compact sets. Let $K \subset B_{b}$ be a compact set. $D_{\delta}$ attracts all points of the Baker domain, so $\bigcup_{n \in \mathbb{N}_{0}} g_{b}^{-n}\left(D_{\delta}\right) \supset B_{b}$. This union covers $K$, and since $K$ is compact, we find a finite subcovering, say, $\bigcup_{n \in I} g_{b}^{-n}\left(D_{\delta}\right)$, where $I \subset\left\{0, \ldots, n_{\max }\right\}$ is a finite index set. But this means that $g_{b}^{n_{\max }}(K) \subset D_{\delta}$.

This finishes the proof of Theorem 1, and we will prove the second statement of Proposition 1 in a more general setting in section 4.2 .

\subsubsection{Singular values}

\section{Proof of Lemma 1:}

The first statement will be proved in a more general context in section 4.2 , the second statement is a consequence of a result of Lyubich and Eremenko mentioned in the introduction.

Let us define

$$
\begin{aligned}
M_{c} & =\left\{z:\left|g_{b}^{\prime}(z)-1\right|<1\right\} \\
& =\left\{x+i y: y^{2}<\frac{e^{-2 x}}{|b|^{2}}-(x+1)^{2}\right\} \\
& =\left\{t-1+i y: y^{2}<e^{2} \frac{e^{-2 t}}{|b|^{2}}-t^{2}\right\} .
\end{aligned}
$$

The last equation shows that $M_{c}$ is just a translation by one of a set we dealt with in Lemma 13, so we obtain analogous results as for the set $M_{c}$ above:

\section{Lemma 14}

$M_{c}$ is non-empty, symmetric with respect to the real axis, consists of at most two components and exactly one component is unbounded. If there are two components, then the bounded one is contained in the strip

$\{z:|\operatorname{Im} z| \leq 1 / 2\}$. If $z \in M_{c}$ and $\operatorname{Im} z>1 / 2$, then $x+i y \in M_{c}$ for all $x, y$ satisfying $x<\operatorname{Re} z,|y|<\operatorname{Im} z$. Furthermore, if $x \in M_{c}^{C} \cap(-1, \infty)$, then $M_{c} \subset\{z: \operatorname{Re} z<x\}$. 
Note that a point $z \in M_{c}$ satisfies $g_{b}^{\prime}(z) \in D(1,1)$, in particular,

$\operatorname{Re} g_{b}^{\prime}(z)>0$ and $\left|\operatorname{Im} g_{b}^{\prime}(z)\right|<1$. Obviously, all critical point of $g_{b}$ are located in $\partial M_{c}$.

We have the following estimations for the critical values:

Lemma 15 (critical values)

Let $z=x+i y \in \mathbb{C}$ be a critical point of $g_{b}$. Then

a) $b e^{z}=-\frac{1}{z+1}$

b) $g_{b}(z)=z-2-b e^{z}=z-2+\frac{1}{z+1}=z-2+\frac{x+1}{|z+1|^{2}}-i \frac{y}{|z+1|^{2}}$

c) $|y|>1.5 \Rightarrow g_{b}(z) \in D(z-2,2 / 3)$ and

d) $y>0 \Rightarrow \operatorname{Im} g_{b}(z)<y$.

\section{Proof:}

a)

$$
\begin{aligned}
& g_{b}^{\prime}(z) \\
\Rightarrow 1+b(z+1) e^{z} & =0 \\
\Rightarrow b e^{z} & =-\frac{1}{z+1} .
\end{aligned}
$$

b)

$$
\begin{aligned}
g_{b}^{\prime}(z) & =0 \\
\Rightarrow b z e^{z} & =-1-b e^{z} \\
\Rightarrow g_{b}(z) & =z-1+b z e^{z} \\
& =z-2-b e^{z} \\
& =z-2+\frac{1}{z+1} .
\end{aligned}
$$

c)

$$
\begin{aligned}
& \begin{aligned}
|y| & >1.5 \\
\Rightarrow|z+1| & >1.5
\end{aligned} \\
& \Rightarrow g_{b}(z) \quad=z-2+\frac{1}{z+1} \in D(z-2,2 / 3)
\end{aligned}
$$

by b).

d)

This is an immediate consequence of $b$ ).

Now we are ready to deal with the critical points. Our general method to show that a critical point is contained in the Baker domain will be to construct an invariant open set intersecting the Baker domain and containing this critical point. The general idea of the construction is described in the following proposition: 


\section{Proposition 8}

Let $z=x+i y$ be a critical point of $g_{b}$ in the upper half plane, $x_{0}<x, \operatorname{Im} g_{b}\left(\tilde{x}+i y_{0}\right)>y_{0}$ for some $y_{0}<y$ and all $\tilde{x}<x$. If the to the left unbounded rectangle $R$ given by the points $x_{0}+i y$ and $x_{0}+i y_{0}$ is contained in $M_{c} \cap M_{1}$, then it satisfies $g_{b}(\bar{R}) \subset R$. The analogous statement is true for all critical points in the lower half plane. Furthermore, if $g_{b}(z) \in R$ and all points $v$ contained in the segment $S=\left\{t+i y: x_{0} \leq t \leq x\right\}$ satisfy Re $g_{b}(v)<\operatorname{Re} g_{b}(z)$, then $g_{b}(S) \subset R$.

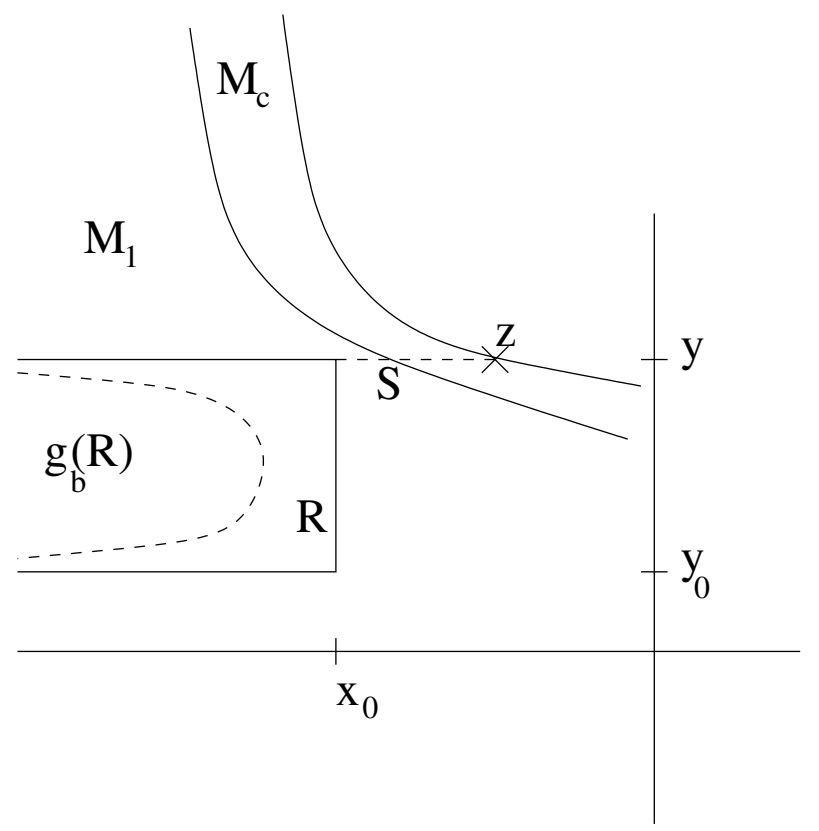

\section{Proof:}

The hypothesis implies that $\operatorname{Re} g_{b}(z)<\operatorname{Re} z$ for all $z \in R \subset M_{1}$. Furthermore, we have $\operatorname{Im} g_{b}(\tilde{x}+i y)<y$ (Lemma 22) and $\operatorname{Im} g_{b}\left(\tilde{x}+i y_{0}\right)>y_{0}$ for all $\tilde{x}<x$. Together with $\operatorname{Re} g_{b}^{\prime}(z)=\frac{d \operatorname{Im} g_{b}(x+i y)}{d y}>0$ on $R \subset M_{c}$, this implies the first claim. The second claim is again a consequence of Lemma 22.

Due to the relations $g_{b}(\bar{z})=\overline{g_{\bar{b}}(z)}$ and $g_{b}^{\prime}(\bar{z})=0 \Leftrightarrow g_{\bar{b}}^{\prime}(z)=0$ we may restrict to the case $\operatorname{Im} b \geq 0$ while examining the forward orbits of the critical points. We will do so in our proofs. 
The critical points are determined by the equation

$$
\begin{array}{rlrl} 
& g_{b}^{\prime}(z) & = & 0 \\
\Leftrightarrow 1+b(z+1) e^{z} & =0 \\
\Leftrightarrow b & = & \frac{-e^{-z}}{z+1},
\end{array}
$$

so the function $h(z)=-\frac{e^{-z}}{z+1}$ maps critical points of $g_{b}$ to the parameter $b$. $h^{\prime}(z)=-\frac{-e^{-z}(z+1)-e^{-z}}{(z+1)^{2}}=\frac{z+2}{(z+1)^{2}} e^{-z}$, so the singularities of the inverse function of $h$ are the critical value $e^{2}$ and the asymptotic value 0 .

$\mathbb{C} \backslash \mathbb{R}_{0}^{+}$is simply connected, so all branches of $h^{-1}$ are defined and holomorphic in this domain and each of these branches maps the parameter value to some critical point of the corresponding function. If we take a parameter $b_{0}$ in $\mathbb{R}^{-}$close to 0 , then the intersection of the boundary of $M_{c}$ and the upper half plane is the graph of a monotonous function. Some elementary calculations give that the critical points are symmetric with respect to the real axis (for this real parameter) and that $\mathbb{R}$ contains exactly one critical point. We may denote the critical points with $z_{n}\left(b_{0}\right), n \in \mathbb{Z}$, such that $\operatorname{Im} z_{n}\left(b_{0}\right)>\operatorname{Im} z_{m}\left(b_{0}\right)$ if $m<n, m, n \in \mathbb{Z}$, and $z_{0}\left(b_{0}\right) \in \mathbb{R}$. By our previous observations we can extend $z_{n}(b)$ to analytic functions on $\mathbb{C} \backslash \mathbb{R} 0_{0}^{+}$for each $n \in \mathbb{Z}$.

Lemma 16 (critical points)

Let $b \in \mathbb{C} \backslash \mathbb{R}_{0}^{+}$. Then

1. $\operatorname{Im} z_{n}(b)>\pi$ if $n>0, \operatorname{Im} b \geq 0$

2. $\operatorname{Im} z_{-n}(b)<0$ if $n>0$

3. $\operatorname{Im} z_{0}(b)>0$ if $\operatorname{Im} b>0$

4. $\operatorname{Im} z_{0}(b) \in(-\pi, \pi)$

5. $\operatorname{Im} z_{n}(b)>\operatorname{Im} z_{m}(b)$ if $n>m$

6. $\left|\operatorname{Im} z_{n}(b)-\operatorname{Im} z_{n+1}(b)\right|$ and $\left|z_{n}(b)-z_{n+1}(b)\right|$ tend to $2 \pi$ as $|n|$ tends to $\infty$. 


\section{Proof:}

We begin with observing that if $\operatorname{Im} b \geq 0$, then the horizontal line at $i \pi$ does not contain a critical point:

$$
\begin{aligned}
g_{b}^{\prime}(x+i \pi) & =0 \\
\Leftrightarrow 1+b(x+1+i \pi) e^{x+i \pi} & =0 \\
\Leftrightarrow b & =\frac{e^{-x}}{x+1+i \pi}=e^{-x}\left(\frac{x+1}{|x+1+i \pi|^{2}}-i \frac{\pi}{|x+1+i \pi|^{2}}\right) \\
\Rightarrow \operatorname{Im} b & <0 .
\end{aligned}
$$

Now the first statement follows from observing that the strip

$\{z: 0<\operatorname{Im} z \leq \pi\}$ does not contain critical points if $b \in \mathbb{R}^{-}$(as done in Lemma 25) and the continuity of the functions $z_{n}$. Our calculations also shows that $\operatorname{Im} z_{0}(b)<\pi$ if $\operatorname{Im} b \geq 0$.

Next, if $x \in \mathbb{R}$ is a critical point, then $1+b(x+1) e^{x}=0$, so $b \in \mathbb{R}$ as well. If $b \in \mathbb{R}^{-}$, then $z_{0}(b) \in \mathbb{R}$, and since $\mathbb{R}$ contains only one critical point we have $\operatorname{Im} z_{n}(b)<0$ for all $n<0$. This proves statement 2 .

To see the third statement it suffices to show that there exists a parameter $b$ on the upper half plane such that the line at $i \frac{\pi}{2}$ contains a critical point. Let $x \in \mathbb{R}$, then $b=\frac{-e^{-x-i \pi / 2}}{1+x+i \pi / 2}$ satisfies $g_{b}^{\prime}(x+i \pi / 2)=1+\frac{-e^{-x-i \pi / 2}}{1+x+i \pi / 2}(x+1+i \pi / 2) e^{x+i \pi / 2}=0$.

By 1 . and 2 . it has to be the critical point $z_{0}(b)$. Hence, $z_{0}(b)$ is contained in the strip bounded by the real axis and the horizontal line at $i \pi$ if $\operatorname{Im} b>0$, by symmetry it is always contained in the strip bounded by the horizontal lines at $i \pi$ and $-i \pi$ if $b \in \mathbb{C} \backslash \mathbb{R}_{0}^{+}$.

To show the fifth statement for $\operatorname{Im} b \geq 0$ and $\left|\operatorname{Im} z_{n}\right|>1 / 2$ or $\left|\operatorname{Im} z_{m}\right|>1 / 2$ we note that two critical points never collide for a parameter outside $\mathbb{R}^{+}$, otherwise some functions $z_{n}(b)$ would not be analytic in this parameter value. Since all critical points move along the boundary of $M_{c}$ which itself moves continuously, this proves the statement outside the strip $\{z:|\operatorname{Im} z|<1 / 2\}$ (note that outside this strip the boundary of $M_{c}$ is the union of the graphs of two continuous and monotonous functions by Lemma 13). Inside the strip a possible problem is that the boundary of $M_{c}$ might no longer be the graph of a monotonous function. But we know that $\operatorname{Im} z_{n}(b)>0$ if $n>0, \operatorname{Im} z_{1}(b)>\pi$ and $\operatorname{Im} z_{-1}(b)<0$, so we only need that $\operatorname{Im} z_{-2}(b)<-2 \pi$. This is true for $b \in \mathbb{R}^{-}$(Lemma 25), and we conclude with showing that the line at $-2 \pi i$ 
does not contain critical points if $\operatorname{Im} b \geq 0$ :

$$
\begin{aligned}
g_{b}^{\prime}(x-2 \pi i) & =0 \\
\Leftrightarrow 1+b(x+1-2 \pi i) e^{x-2 \pi i} & =0 \\
\Leftrightarrow b & =-\frac{e^{-x}}{x+1-2 \pi i} \\
\Rightarrow \operatorname{Im} b & =e^{-x}\left(-\frac{x+1}{|x+1-2 \pi i|^{2}}-i \frac{2 \pi}{|x+1-2 \pi i|^{2}}\right) \\
& <0 .
\end{aligned}
$$

To see the last statement we recall that $\partial M_{c}$ is mapped by $g_{b}^{\prime}$ into $\partial D(1,1)$ and contains all critical points. It suffices to show that $\left|g_{b}^{\prime \prime}(z)\right|$ tends to 1 as $z \in \partial M_{c}$ and $|z|$ tends to $\infty$. We calculate

$\left|g_{b}^{\prime \prime}(z)\right|=\left|b(z+2) e^{z}\right|=\left|b(z+1) e^{z}+b e^{z}\right| \rightarrow 1$ under these conditions.

Theorem 2 and Lemma 2 will be a consequence of the following proposition:

\section{Proposition 9}

a)

If $b \in \mathbb{C} \backslash \mathbb{R}_{0}^{+}$and $n \in \mathbb{Z} \backslash\{0\}$, then there exists a rectangle $R_{n}$ of the form $\left\{z: \operatorname{Re} z<x_{0}, y_{0}<\operatorname{Im} z<y_{1}\right\}$ such that $y_{1}-y_{0}<2 \pi, g_{b}\left(\overline{R_{n}}\right) \subset R_{n}$ and $g_{b}$ is conformal on $R_{n}$. Furthermore, we can take $R_{n}$ such that $R_{n}$ contains $g_{b}\left(z_{n}(b)\right)$ and there is a segment connecting $z_{n}(b)$ with $\partial R_{n}$ which is mapped into $R_{n}$ as well.

b)

If $b \in \mathbb{R}^{+} \cup D(0,0.1) \backslash\{0\}$, then all critical points of $g_{b}$ are contained in the Baker domain $B_{b}$.

\section{Proof:}

a)

Again, we may restrict to the case $\operatorname{Im} b \geq 0$. Let $b=c+i d, c \in \mathbb{R}$ and $d \in \mathbb{R}^{+}$. For $b \in \mathbb{R}^{-}$the $\operatorname{strip}\{z: 0<\operatorname{Im} z \leq \pi\}$ does not contain critical points (see Lemma 25), so $\operatorname{Im} z_{n}(b)>y_{+}(b)=\pi$ for $n>0$ and by symmetry $\operatorname{Im} z_{n}(b)<y_{-}(b)=-\pi$ for $n<0\left(y_{+}(b)\right.$ and $y_{-}(b)$ are defined in Lemma 27). Since $\operatorname{Im} g_{b}\left(z_{n}\right)<\operatorname{Im} z_{n}(b)$ for all $n>0$, we have $\operatorname{Im} z_{1}(b) \neq y_{+}(b)$ for all $b \in \mathbb{C} \backslash \mathbb{R}_{0}^{+}$, so continuity gives that $\operatorname{Im} z_{n}(b)>y_{+}(b)$ for all $b \in \mathbb{C} \backslash \mathbb{R}_{0}^{+}$and $n>0$. Hence, for each critical point $z_{n}(b), n>0$, the value $y_{n}^{+}(b)=\max _{y<\operatorname{Im} z_{n}(b)}\left\{y=y_{+}(b)+2 \pi k, k \in \mathbb{N}_{0}\right\}>0$ is well defined. Analogously, we obtain a value $y_{n}^{-}(b)=\min _{y>\operatorname{Im} z_{n}(b)}\left\{y=y_{-}(b)-2 \pi k, k \in \mathbb{N}_{0}\right\}<0$ for each $z_{n}(b), n<0$. In Lemma 27 we calculate some properties of $y_{n}^{+}(b)$ and $y_{n}^{-}(b)$.

First, let $n>0$.

Combining Lemma 23 and Proposition 8 gives that the to the left unbounded 
rectangle $R_{n}(b)$ given by $z_{n}(b)-4 / 3$ and $\operatorname{Re} z_{n}(b)-4 / 3+i y_{n}^{+}(b)$ is forward invariant. The same proposition gives that the segment connecting $z_{n}$ and $z_{n}-\frac{4}{3}$ is mapped into $R_{n}$ as well (note Lemma 15c) and Lemma 22b)1. for that). To see the conformality we note that the boundary of $R_{n}$ is mapped univalently to a Jordan curve. This is a consequence of $\frac{d \operatorname{Im} g_{b}\left(x+i \operatorname{Im} z_{n}(b)\right)}{x}<0$ for $x<\operatorname{Re} z_{n}(b)($ Lemma 22$), \frac{d \operatorname{Im} g_{b}\left(\operatorname{Re} z_{n}(b)-\frac{4}{3}+i y\right)}{x}>0$ for $y \in\left(y_{n}^{+}, \operatorname{Im} z_{n}(b)\right)$ and $\frac{d \operatorname{Im} g_{b}\left(x+i \operatorname{Im} z_{n}(b)\right)}{x}>0$ for $x \in \mathbb{R} \quad($ Lemma 27$)$.

The case $n<0$ is only slightly more difficult. As we fix a parameter $b$ we will drop $b$ in our notations from now on.

Let $n<0, z_{n}=x_{n}+i y_{n}$ be a critical point of $g_{b}$, let $L_{1}$ be the line $\left\{x+i y_{n}^{-}: x \leq x_{n}-4 / 3\right\}, L_{2}$ the segment connecting $x_{n}-4 / 3+i y_{n}^{-}$and $z_{n}-4 / 3$ and $L_{3}$ the line $\left\{z+i y_{n}: x \leq x_{n}-4 / 3\right\}$. We will show that the rectangle $R$ bounded by $L_{1} \cup L_{2} \cup L_{3}$ is forward invariant with respect to $g_{b}$. First, let $y_{n} \leq-2 / 3$. By Lemma $23, R$ is contained in $M_{1} \cup M_{c}$ and we are done by applying proposition 8 once more. Combining Proposition 8 , Lemma 22 and Lemma 15c), respectively, gives that $z_{n} \in B_{b}$ as well.

If $y_{n}>-2 / 3$ then we prove that the to the left unbounded rectangle $\tilde{R}$ given by $z_{n}$ and Re $z_{n}+i y_{n}^{-}$is mapped into $R$. Note that Lemma 24 gives $x_{n}=\operatorname{Re} z_{n}<-1$. The segment $S$ connecting $z_{n}$ and $x_{n}+i y_{n}^{-}$is contained in $M_{c} \cup\left\{z_{n}\right\}$, so we have $\operatorname{Im} g_{b}\left(z_{n}\right)<\operatorname{Im} g_{b}\left(x_{n}+i y\right)<\operatorname{Im} g_{b}\left(x_{n}+i y_{n}^{-}\right)$for $y_{n}^{-}>y>y_{n}$. Again using $S \subset M_{c} \cup\left\{z_{n}\right\}$ we obtain $\left|\operatorname{Im} g_{b}^{\prime}(z)\right|<1$ for all $z \in S$, so

$$
\begin{aligned}
\operatorname{Re} g_{b}\left(x_{n}+i y\right) & <\operatorname{Re} g_{b}\left(z_{n}\right)+2 / 3 \\
& =x_{n}-2+\operatorname{Re} \frac{1}{z_{n}+1}+2 / 3<x_{n}-2+2 / 3 \\
& =x_{n}-4 / 3,
\end{aligned}
$$

therefore $S$ is mapped into $R$.

To see that the line $\left\{x+i y_{n}^{-}: x \leq x_{n}\right\}$ is mapped into $R$ we note that $\operatorname{Im} g_{b}\left(x+i y_{n}^{-}\right)<y_{n}^{-}$and $\frac{d}{d x} \operatorname{Re} g_{b}\left(x+i y_{n}^{-}\right)>0$ for all $x<x_{n}$ (Lemma 27, 6.), so $\operatorname{Re} g_{b}\left(x+i y_{n}^{-}\right)<\operatorname{Re} g_{b}\left(x_{n}+i y_{n}^{-}\right)<x_{n}-\frac{4}{3}$.

The fact that the line $\left\{x+i y_{n}: x \leq x_{n}\right\}$ is mapped into $R$ is a consequence of $x_{n}<-1, \operatorname{Re} g_{b}\left(z_{n}\right)=x_{n}-2+\frac{x_{n}+1}{\left|z_{n}\right|^{2}}<x_{n}-2$ and Lemma 22a) and $\mathrm{b}$ ).

The conformality can be seen as above.

b)

Let $b \in \mathbb{R}^{+}$. Since $g_{b}$ maps $\mathbb{R}$ into itself, the set of critical points and the Julia set are symmetric with respect to the real axis.

Let $z=x+i y$ be a critical point in the upper half plane. Let

$y_{0}=\max _{n \geq 0}\{2 \pi n: 2 \pi n<y\}$, then $\operatorname{Im} g_{b}\left(t+i y_{0}\right)=y_{0}+b y_{0} e^{t} \geq y_{0}$ for all $t \in \mathbb{R}$. First, we look at the case $\operatorname{Im} z \geq \pi / 2$. In this case, Lemma 23 shows 
that the to the left unbounded rectangle given by $z-4 / 3$ and $\operatorname{Re} z-4 / 3+i y_{0}$ satisfies the hypothesis of Proposition 8. Thus, it is contained in the Baker domain $B_{b}$, and as before we see that the critical point $z$ is contained in $B_{b}$ as well.

If $\operatorname{Im} z<\pi / 2$, then we calculate

$\operatorname{Re} g_{b}(z)=\operatorname{Re}\left(x-2-b e^{z}\right)=x-2-b \cos y e^{x}<x-2$.

The segment $S$ connecting $z$ and $\operatorname{Re} z$ is contained in $\overline{M_{c}}$, by definition of $M_{c}$ we have $\left|\operatorname{Im} g_{b}^{\prime}(v)\right|=\left|\frac{d \operatorname{Re} g_{b}(v)}{d \operatorname{Im} v}\right|<1$ for all $v \in M_{c}$, so $\operatorname{Re} g_{b}(v)<\operatorname{Re} v$ for all $v \in S$. Furthermore, $\operatorname{Im} g_{b}^{\prime}(x+i y)=b((x+1) \sin y+y \cos y) e^{x}$, so $x<-1$. For each $t \in \mathbb{R}^{-}$we have $g_{b}(t)<t-1$, so the interval $(-\infty, t)$ is invariant. Now we can apply Lemma 22 as before and conclude that the boundary of the to the left unbounded rectangle $R$ given by $z$ and $\operatorname{Re} z$ is mapped into $\bar{R}$, so $R$ is invariant.

It remains to look at critical points on the real line. If $|b| \geq 1$, then $\frac{e^{0}}{|b|^{2}}-(1)^{2} \leq 0$, so every real critical point is contained in $\mathbb{R}_{0}^{-}$(Lemma 14). All points $t \in \mathbb{R}_{0}^{-}$satisfy $g_{b}(t) \leq t-1$. Thus, all real critical points are contained in the Baker domain.

If $|b|<1$, then $g_{b}^{\prime}(x)=1+b(x+1) e^{x}>1+1\left(-e^{-2}\right)>0$ for all $x \in \mathbb{R}$, so there are no real critical points.

Now we will examine the punctured disk $D(0,0.1) \backslash\{0\}$.

Let $b \in D(0,0.1) \backslash\{0\}$. By a), symmetry arguments and our results on $\mathbb{R}^{+}$we only need to consider the critical point $z_{0}=z_{0}(b)=x+i y$ for $b$ in the upper half plane and the real critical points for $b \in \mathbb{R}^{-}$. First, let $b$ be in the upper half plane $H^{+}$. We already know that $\operatorname{Im} z_{0} \in[0, \pi)$, and the reasoning, following the ideas used in a), is as follows: first, we see that $\frac{e^{-6 / 4}}{|b|^{2}}-(1+3 / 4)^{2}>\pi^{2}$, so $x>3 / 4$. This yields $\left|b e^{z_{0}}\right|^{-1}=\left|z_{0}+1\right|>7 / 4$, so $\operatorname{Re} g_{b}\left(z_{0}\right)=x-2-\operatorname{Re} \frac{1}{z_{0}+1}<x-2$ and $\operatorname{Im} g_{b}\left(z_{0}(b)\right)>y-\frac{7}{4}>-\frac{7}{4}$. As in Lemma 27 we can find a $y^{+} \in\left[-2 \pi-\frac{7}{4},-\frac{7}{4}\right)$ such that $\operatorname{Im} g_{b}\left(t+i y^{+}\right)>y^{+}$ for all $t \in \mathbb{R}$. We seek to show that the rectangle given by $z_{0}(b)-2$ and $x-2+i y^{+}$is forward invariant. In order to be able to apply Lemma 22 and Proposition 8 we only need to show that Re $z_{0}(b)-2-2 \pi-\frac{7}{4} \in M_{1} \cap M_{c}$, and to do this we show that

$$
\frac{e^{-2(x-2)}}{|b|^{2}}-(x-2)^{2}>\left(2 \pi+\frac{7}{4}\right)^{2} \text { and } \frac{e^{-2(x-2)}}{|b|^{2}}-(x-2+1)^{2}>\left(2 \pi+\frac{7}{4}\right)^{2} .
$$

Since $x>3 / 4>1 / 2$, the second inequation implies the first one, and 


$$
\begin{aligned}
& \frac{e^{-2(x-2)}}{|b|^{2}}-(x-2+1)^{2} \\
= & \left(e^{4}-1\right) \frac{e^{-2 x}}{|b|^{2}}+\frac{e^{-2 x}}{|b|^{2}}-(x+1)^{2}+4 x \\
> & \left(e^{4}-1\right) \frac{49}{16}+y^{2}+4 \frac{3}{4} \\
> & \left(2 \pi+\frac{7}{4}\right)^{2},
\end{aligned}
$$

and we may conclude as before.

It remains to look at the real critical points of parameters in $(-0.1,0)$. If $b \in(-0.1,0)$, then for any $x \in \mathbb{R}$ we have $b x e^{x}<b e^{-1}$ yielding $g_{b}(x)<x-1+b e^{-1}<x-1+e^{-1} / 10$. Hence, $\mathbb{R} \in B_{b}$.

Note that $-1+\frac{3 \pi i}{2}$ is a critical point of $g_{b_{0}}$, where $b_{0}=-\frac{2 e}{3 \pi}$. Furthermore, $y_{+}\left(-\frac{2 e}{3 \pi}\right)=\pi$ and the strip $\{z:|\operatorname{Im} z| \leq \pi\}$ does not contain critical points, so $z_{1}\left(-\frac{2 e}{3 \pi}\right) \in\left(y_{+}\left(-\frac{2 e}{3 \pi}\right), y_{+}\left(-\frac{2 e}{3 \pi}\right)+\pi\right)$. Since

$$
\begin{aligned}
g_{b}\left(z_{1}(b)+1\right) & =z_{1}(b)+1-1+b\left(z_{1}(b)+1\right) e^{z_{1}(b)+1} \\
& =z_{1}(b)+e b\left(z_{1}(b)+1\right) e^{z_{1}(b)} \\
& =z_{1}(b)-e+e g_{b}^{\prime}\left(z_{1}(b)\right) \\
& =z-e
\end{aligned}
$$

we obtain $z_{1}(b) \neq y_{+}(b)$ and $z_{1}(b) \neq y_{+}(b)+\pi$ for all $b \in\{z: \operatorname{Im} z>0\} \cup \mathbb{R}^{-}$. We can proceed analogously for $z_{-1}(b)$ and obtain

\section{Corollary 7}

$R_{1}$ is contained in the strip

$\left\{z: y_{+}(b)<\operatorname{Im} z<y_{+}(b)+\pi\right\} \subset\{z: 0<\operatorname{Im} z<3 \pi\}$ and $R_{-1}$ is contained in $\left\{z: y_{-}(b)>\operatorname{Im} z>y_{-}(b)-\pi\right\} \subset\{z: 0>\operatorname{Im} z>-3 \pi\}$.

\section{Remark 15}

Doing things more carefully, one may prove that for all parameters $b$ satisfying $0<|b|<1 / 4$ all critical points of $g_{b}$ are contained in the Baker domain $B_{b}$.

The last information we need to know about critical points is if there are multiple critical points.

\section{Lemma 17}

If $g_{b}$ has a multiple critical point, then $b=e^{2}$.

\section{Proof:}

One obtains this result by solving the systems of two equations given by $g_{b}^{\prime}(z)=g_{b}^{\prime \prime}(z)=0$ for $b$ (analogously to the proof of Lemma 21). 


\subsubsection{Components and geometry of the Fatou set}

\section{Proof of Proposition 4:}

We will use the rectangles $R_{k}, k \in \mathbb{Z} \backslash\{0\}$, as constructed in Proposition 9 . i)

Again, it suffices to do this for $\operatorname{Im} b \geq 0$. In section 4.1.1 we labelled all critical points of $g_{b}$ with $z_{k}, k \in \mathbb{Z}$, for all $b \in \mathbb{C} \backslash \mathbb{R}_{0}^{+}$. Using continuity arguments we can do this for all non-real critical points of $g_{b}, b \in \mathbb{R}^{+}$, as well. Let $k \in \mathbb{Z} \backslash\{0\}$, let $v_{k}=g_{b}\left(z_{k}\right), w_{k}=g_{b}\left(v_{k}\right)$ and $L_{k}$ be the segment connecting $v_{k}$ and $w_{k}$.

Then $\gamma_{k}:=O^{+}\left(g_{b}\left(L_{k}\right)\right)$ is contained in a to the left unbounded horizontal rectangle $R_{k}$ as was stated in Corollary 7 . Since $z_{k}$ is a simple critical point, there is exactly one curve $\Gamma_{k}$ in $g_{b}^{-1}\left(\gamma_{k}\right)$ such that $\Gamma_{k}$ is mapped 2:1 to $\gamma_{k}$ and $\gamma_{k} \subset \Gamma_{k}$.

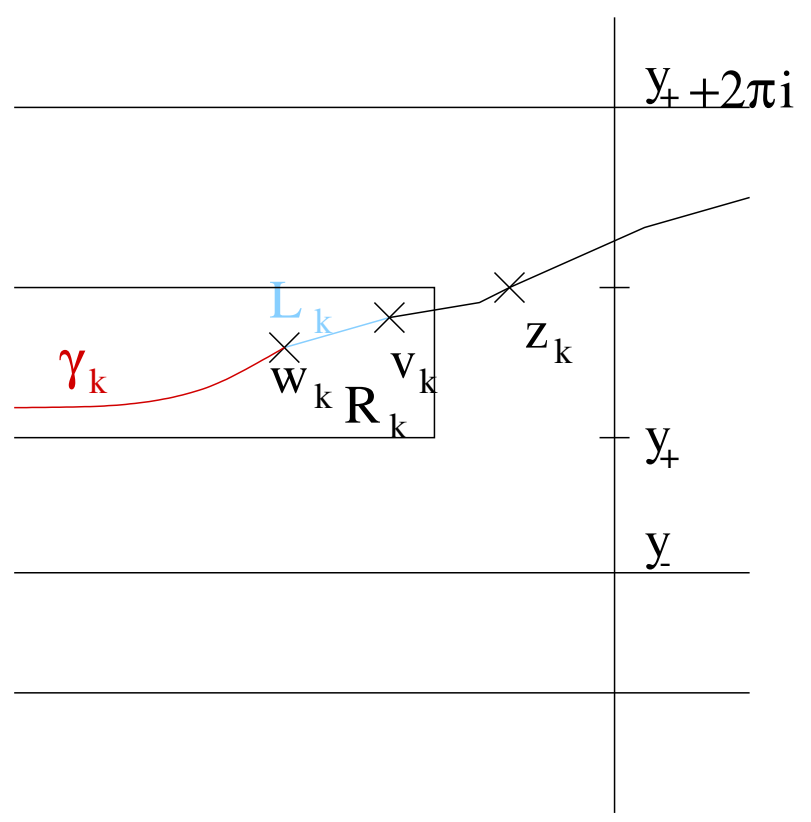

Obviously, $\Gamma_{k} \subset B_{b}$. Proposition 9 gives that $\Gamma_{n}$ and $\Gamma_{m}$ are disjoint for $m \neq n$.

If $k>0$, then the lower boundary of $R_{k}$ contains a line of the form $\left\{t+i y_{k}^{+}: t \leq \operatorname{Re} z_{k}\right\}$, where $y_{k}^{+}$has the property that $\operatorname{Im} g_{b}\left(x+i y_{k}^{+}\right)>y_{k}^{+}$ for all $x \in \mathbb{R}$ (see Lemma 27). If $z \in\left\{t+i\left(y_{k}^{+}+2 \pi\right): t \in \mathbb{R}\right\}$, then $\operatorname{Im} g_{b}(z)>y_{k}^{+}+2 \pi$, so $z \notin \Gamma_{k}$. As in Lemma 27 we find an $y_{k}^{-} \in\left(y_{k}^{+}-3 \pi, y_{k}^{+}\right)$ such that $\operatorname{Im} g_{b}(z) \leq y_{k}^{-}$if $\operatorname{Im} z=y_{k}^{-}$. Hence, $\Gamma_{k}$ is contained in the strip $\left\{z: y_{k}^{+}-3 \pi<\operatorname{Im} z<y_{k}^{+}+2 \pi\right\}$. Now suppose that the set $\left\{\operatorname{Re} z: z \in \Gamma_{k}\right\}$ 
is bounded above. Let $U$ be a domain bounded by $\Gamma_{k}$ not containing a half plane. Then $g_{b}(\partial U)=\gamma_{k}$, so the image of $U$ contains a half plane. But the image of a set of the form $\left\{z: y_{0}>\operatorname{Im} z>y_{0}-5 \pi\right.$, Re $\left.z<c\right\}$, where $y_{0}, c \in \mathbb{R}$, cannot contain a half plane due to our results on the Baker domain. Thus, $\Gamma_{k}$ stretches to " $+\infty "$. Note that $\gamma_{1} \subset R_{1}(b) \subset\{z: \operatorname{Im} z>\pi\}$ and $z_{0}(b)$ is always contained in the strip bounded by $\Gamma_{1}$ and $\Gamma_{-1}$ if $b \in \mathbb{R}_{0}^{+}$. If $b \in \mathbb{R}^{+}$, then all real critical points (which are at most two) are contained in this strip.

Let $k>0$ and $S_{k}$ the set bounded by $\Gamma_{k}$ and $\Gamma_{k+1} . \partial S_{k}$ is forward invariant and $S_{k}$ does not contain a critical point. Define $\tilde{S}_{k}=S_{k} \backslash g_{b}^{-1}\left(\Gamma_{k} \cup \Gamma_{k+1}\right)$. $S_{k} \backslash \tilde{S}_{k}$ is a finite union of disjoint Jordan arcs, so $\tilde{S}_{k}$ is simply connected. The boundary of $\tilde{S}_{k}$ is mapped to $\gamma_{k} \cup \gamma_{k+1}$, and the Open Mapping Theorem gives $\partial g_{b}\left(\tilde{S}_{k}\right)=\gamma_{k} \cup \gamma_{k+1}$, so $g_{b}\left(\tilde{S}_{k}\right)=\mathbb{C} \backslash\left(\gamma_{k} \cup \gamma_{k+1}\right)$ is simply connected as well. Furthermore, $g_{b \mid \tilde{S}_{k}}$ is a proper mapping of finite degree. By the RiemannHurwitz-Formula $g_{b \mid \tilde{S}_{k}}$ is univalent. This in turn implies $S_{k} \backslash \tilde{S}_{k}=\emptyset$, so $g_{b \mid S_{k}}$ is a conformal mapping from $S_{k}$ onto $\mathbb{C} \backslash\left(\gamma_{k} \cup \gamma_{k+1}\right)$. We obtain the analogous result for $-k$ and the strip bounded by $\Gamma_{-k}$ and $\Gamma_{-k-1}$.

ii)

Let $D=D_{0.5, R}$ be a fundamental domain as given by Theorem 1 . We may assume that $0 \notin \bar{D}$ (otherwise we take a $\tilde{R}<R$ such that this is true). $D$ is of the form $\left\{x+i y: y^{2}<\frac{1}{4} \frac{e^{-2 x}}{|b|^{2}}-x^{2}, x<-R\right\}$. All critical points are located on the curve $\partial M_{c}=\left\{x+i y: y^{2}=\frac{e^{-2 x}}{|b|^{2}}-(x+1)^{2}\right\}$. The asymptotic distance in the sense of Lemma 29 between $\partial M_{c}$ and $\partial D$ is $|\ln 0.5|=\ln 2$. Every critical point satisfies $g_{b}(z)=z-2+\frac{1}{z+1}$. If $|z|$ is sufficiently large then $g_{b}(z) \in D$, so $D$ contains all but finitely many critical values. Furthermore, all but finitely many curves $\gamma_{n}, n \in \mathbb{Z} \backslash\{0\}$, as constructed in i) are contained in $D$. Let $I$ be the index set such that $D$ contains $\gamma_{n}$ if and only if $n \in I$, then $d\left(\bigcup_{i \in I} \gamma_{i}, \partial B_{b}\right)>\sigma$ for some $\sigma>0$. Let $K>0$ such that the translation of $D$ by $K$ contains the disk $D(0,2)$ and $\frac{1}{2}\left|b v e^{v}\right|\left|\frac{v+x}{v}\right| e^{x}-x-10 \pi-1>0$ for all $x \in(K, \infty)$ and $v \in \partial D$ (note that $\left|b v e^{v}\right|$ is bounded away from 0 on $\partial D$ ). Let $D_{K}$ be the translation of $D$ by $K$. Then

$$
\begin{aligned}
\left\|g_{b}^{\prime}\right\|_{\overline{D_{K}}} & =\sup _{z \in D_{K}}\left|1+b(z+1) e^{z}\right| \\
& =\sup _{z \in D_{K}}\left|1+b(z-K) e^{z-K} e^{K}+b(K+1) e^{z}\right| \\
& \leq 1+\sup _{z \in D} e^{K}\left|b z e^{z}\right|+\sup _{z \in D_{K}}\left|b(K+1) e^{z}\right| \\
& =1+\frac{e^{K}}{2}+\sup _{z \in D_{K}}\left|b(K+1) e^{z}\right|<\infty .
\end{aligned}
$$

Let $\Gamma_{k}$ be the component of $g_{b}^{-1}\left(\gamma_{k}\right)$ as in i), then we can find an $\varepsilon_{1}>0$ such 
that $\varepsilon_{1}$-neighborhood of $D_{K} \cap \bigcup_{i \in I} \Gamma_{i}$ is contained in $B_{b}$.

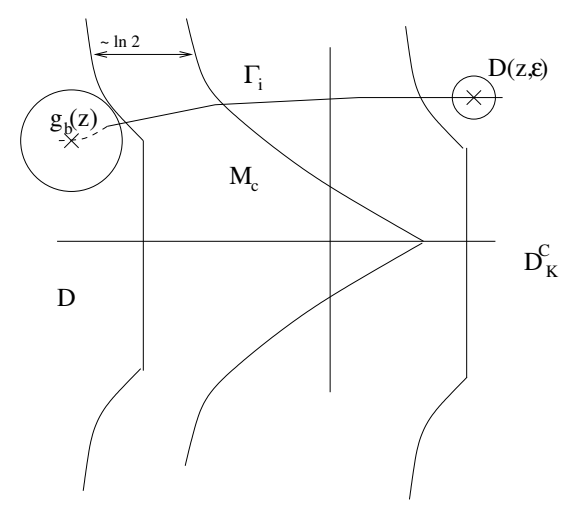

We are done if we show that for some $\varepsilon>0$, all $i \in I$ and $z \in D_{K}^{C} \cap \Gamma_{i}$ we have $\left.\varepsilon|| g_{b}^{\prime}\right|_{\overline{D(z, \varepsilon)}}<d\left(g_{b}(z), \partial D\right)$. Let $\varepsilon>0$ such that $2 \varepsilon e^{\varepsilon}\left|b z e^{z}\right|+\varepsilon<\frac{1}{4}\left|b z e^{z}\right|$ for all $z \in D_{K}^{C}$. Let $i \in I, z \in D_{k}^{C} \cap \Gamma_{i}, v \in \partial D \cap\left\{x+i \operatorname{Im} g_{b}(z), x \in \mathbb{R}\right\}$ and $\tilde{v} \in \partial D \cap\{x+i \operatorname{Im} z, x \in \mathbb{R}\}$. Using that $g_{b}(z) \in \gamma_{i}$, that each $\Gamma_{i}$ is contained in a horizontal strip of height less than $5 \pi$ and that any tangent to $\partial D$ has a slope of modulus larger than one (cf. the construction of $D$ ) we obtain $d\left(g_{b}(z), \partial D\right)>\frac{1}{2}\left|g_{b}(z)-v\right|$ and $|v-z|<|z-\tilde{v}|+10 \pi=: x+10 \pi$, where $x \in(K, \infty)$. Hence,

$$
\begin{aligned}
& d\left(g_{b}(z), \partial D\right) \\
> & \frac{1}{2}\left|g_{b}(z)-v\right| \\
\geq & \frac{1}{2}\left(\left|g_{b}(z)-z\right|-|v-z|\right) \\
\geq & \frac{1}{2}\left(\left|b z e^{z}-1\right|-x-10 \pi\right) \\
\geq & \frac{1}{2}\left(\left|b z e^{z}\right|-1-x-10 \pi\right) \\
= & \frac{1}{4}\left|b z e^{z}\right|+\frac{1}{2}\left(\frac{1}{2}\left|b \tilde{v} e^{\tilde{v}}\right|\left|\frac{\tilde{v}+x}{\tilde{v}}\right| e^{x}-1-x-10 \pi\right) \\
\geq & \frac{1}{4}\left|b z e^{z}\right| \\
\geq & 2 \varepsilon e^{\varepsilon}\left|b z e^{z}\right|+\varepsilon \\
\geq & \varepsilon\left(\left|b(z+1+\varepsilon) e^{z+\varepsilon}\right|+1\right) \\
\geq & \varepsilon|| g_{b}^{\prime}||_{D(z, \varepsilon)} .
\end{aligned}
$$

Hence, $D(z, \varepsilon) \subset B_{b}$.

\section{Corollary 8}

Together with Corollary 7 the proof of i) gives that the curves $\Gamma_{1}$ and $\Gamma_{-1}$ are contained in the strip $\{z:-4 \pi<\operatorname{Im} z<4 \pi\}$. 
Another useful corollary is the following.

\section{Corollary 9}

There exists an $\varepsilon>0$ such that $d\left(P(f) \backslash O^{+}\left(z_{0}(b)\right), \partial B_{b}\right)>\varepsilon$.

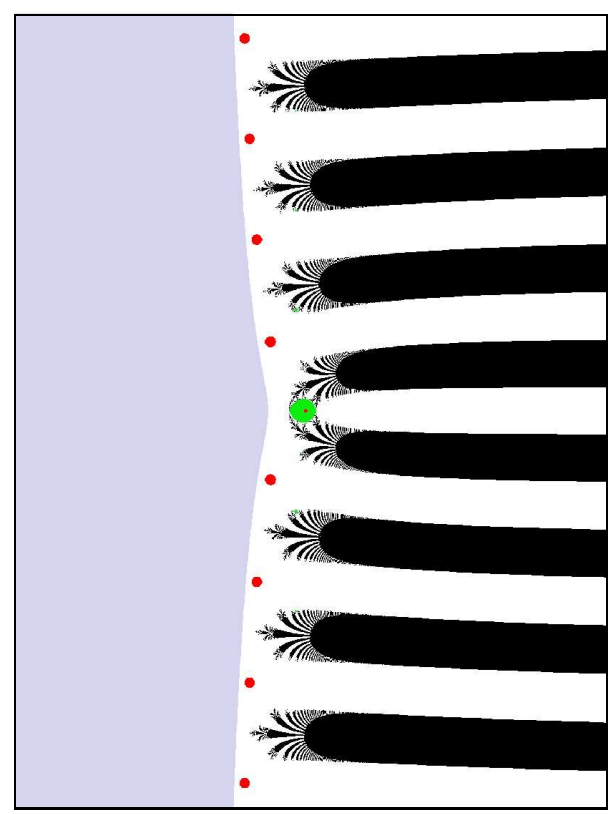

Figure 4.5: The Julia set of $g_{-3}$ is shown in black,the blue set is the absorbing domain as given in the theorem with $\delta=0.5$ and $R=1$, the green set is the attracting Basin of an attracting fixed point. Neighborhoods of the critical points are plotted in red

\section{Proof of Proposition 2:}

Once more we may restrict to $b \in \overline{H^{+}} \backslash\{0\}$. Let $M$ be a bounded set, let $\tilde{M}$ be a bounded, simply connected set containing $M, g_{b}(M)$, a critical point $z$ and $g_{b}(z)$. For $b \in H^{+}$Lemma 27 guarantees the existence of $y_{-}, y_{+} \in \mathbb{R}$ such that $\operatorname{Im} g_{b}\left(x+i y_{-}\right)<y_{-}$and $\operatorname{Im} g_{b}\left(x+i y_{+}\right)>y_{+}$for all $x \in \mathbb{R}$ and $y_{-}<\inf _{z \in \tilde{M}} \operatorname{Im} z$ and $y_{+}>\sup _{z \in \tilde{M}} \operatorname{Im} z$, for $b \in \mathbb{R} \backslash\{0\}$ we can choose such values in the set $\{n \pi: n \in \mathbb{Z}\}$. Furthermore, we can find 
$n>1, x_{-}<0$ and $x_{+}>0$ such that $\tilde{M}$ is contained in the rectangle $R$ given by $x_{-}+i\left(y_{n}^{-}(b)-2 \pi n\right), x_{+}=i\left(y_{n}^{-}(b)-2 \pi n\right), x_{-}+i\left(y_{n}^{-}(b)+2 \pi n\right)$ and $x_{+}+i\left(y_{n}^{-}(b)+2 \pi n\right)$, and such that the segment connecting $x_{-}+i\left(y_{n}^{+}(b)-2 \pi n\right)$ and $x_{+}+i\left(y_{n}^{+}(b)+2 \pi n\right)$ is contained in $M_{1}$ and $\left|g_{b}(z)\right|>\max _{z \in R}|z|$ for all points on the segment connecting $x_{-}+i\left(y_{n}^{+}(b)-2 \pi n\right)$ and $x_{+}+i\left(y_{n}^{+}(b)+2 \pi n\right)$. Then $g_{b}(\partial R)$ does not intersect $\bar{R}$ but encloses $R$. We may assume that $\partial R$ does not contain critical points, otherwise we change $R$ a little but such that this is true and $R$ still has the properties mentioned above. There exists a simply closed curve $\gamma \subset g_{b}(\partial R)$ such that the interior int $\gamma$ of $\gamma$ does not intersect $g_{b}(\partial R)$. Then $g_{b}$ is polynomial-like on the component of $g_{b}^{-1}$ (int $\gamma$ ) which is contained in $R$ and contains $\tilde{M}$.

\section{Proof of Proposition 3:}

Assume that $g_{b}$ has more than one non-repelling cycle. Then we can find a bounded domain $U$ containing two such cycles such that $g_{b}$ is polynomiallike on $U$. This implies the existence of a polynomial $P$ such that all critical points of $P$ except for at most one escape to $\infty$ having two finite non-repelling cycles. Shishikura [50] proved that this is impossible.

By a result mentioned in the introduction all Fatou components are simply connected if an entire function has a Baker domain. Assume that we have an unbounded periodic Fatou component $V \neq B_{b}$ of period $p$. By Proposition 4 we can find an $C>0$ such that $U=\bigcup_{i=0}^{p-1} g_{b}^{i}(V)$ is contained in the strip $\{z:|\operatorname{Im} z|<C\}$. Clearly, the set $\{\operatorname{Re} z: z \in U\}$ is unbounded above, but now we obtain for all $z \in U$ having a sufficiently large real part that $\left(\operatorname{Re} g_{b}(z)\right)^{2}>\left|g_{b}(z)\right|-C^{2} \approx b|z| e^{\operatorname{Re} z} \gg(\operatorname{Re} z)^{2}$, so $g_{b}^{n}(z)$ tends to $\infty$ as $n$ does. By Corollary 10 we already know that this is impossible.

The statement on the number of Baker domains will be proved in the next subsection.

\subsubsection{On the existence of wandering domains}

The following lemma and corollary deal with points escaping to $\infty$.

\section{Lemma 18}

There exists an $\varepsilon>0, R>0$ such that the set $\left\{z:|z|>R,\left|g_{b}^{\prime}(z)\right|<1+\varepsilon\right\}$ is contained in $B_{b}$.

Proof: We will prove a more general statement in section 4.2 . 
Corollary 10 If $g_{b}^{n}(z) \rightarrow \infty$, then $z \in B_{b} \cup J\left(g_{b}\right)$.

\section{Proof:}

Suppose that $g_{b}^{n}(z) \rightarrow \infty$, but $z \notin B_{b} \cup J\left(g_{b}\right)$. Then $z$ is contained in the Fatou set, so we can find a neighborhood of $U$ of $z$ such that all points in $U$ tend to $\infty$ under iteration. By the previous lemma, $\left(g_{b}^{\prime}\right)^{n}(z)$ tends to $\infty$ as well. By Bloch's theorem the images $g_{b}(U)$ contain circles of "exploding" radii. This is impossible, since we have horizontal channels with an bounded width.

This implies that $B_{b}$ is the only Baker domain of $g_{b}$. Moreover, $g_{b}$ has no Baker wandering domains. We also have the following:

\section{Lemma 19}

Let $V$ be a wandering domain of $g_{b}$. Then the free critical point is contained in the Julia set, but not on the boundary of a wandering domain. Moreover, the constant limit function $\infty$ is contained in the set of limit functions, and for each $z \in V$ the set $\left\{\operatorname{Im} g_{b}^{n}(z), n \in \mathbb{N}\right\}$ is unbounded.

\section{Proof:}

First, we prove that the constant function $\infty$ is contained in the set of limit functions.

Let $V$ be a wandering domain of $g_{b}$. If $U=\bigcup_{n \in \mathbb{N}} g_{b}^{n}(V)$ would be bounded then we could find a domain $V \supset U$ such that $g_{b}$ is polynomial-like on $V$, contradicting the fact that polynomials do not have wandering domains. Hence, $U$ is unbounded. Now assume that $U$ is contained in a horizontal strip, say, $U \subset\{z:|\operatorname{Im} z|<C\}$ for some $C>0$, then the set $\{\operatorname{Re} z: z \in U\}$ is unbounded above. But for all $z \in U$ having a sufficiently large imaginary part we obtain $\left(\operatorname{Re} g_{b}(z)\right)^{2}>\left|g_{b}(z)\right|-C^{2} \approx b|z| e^{\operatorname{Re} z} \gg(\operatorname{Re} z)^{2}$ contradicting the fact that $\left\{g_{b \mid V}\right\}_{n \in \mathbb{N}}$ does not converge do the constant function $\infty$. Hence, the set $\left\{\operatorname{Im} g_{b}^{n}(z): z \in V\right\}$ is unbounded, now Proposition 4 implies that $\infty$ is a limit function of $\left\{g_{b \mid V}\right\}_{n \in \mathbb{N}}$.

Again by Proposition 4 we have curves $\Gamma_{n}, n \in \mathbb{Z}$, separating the plane into approximately horizontal strips. Moreover, we can find an $\varepsilon>0$ such that the $\varepsilon$-neighborhood of all $\Gamma_{n}$ is contained in the Baker domain for all but finitely many $n$. We keep the notation of proposition 4 and denote the strips with $S_{n}, n \in \mathbb{Z}$, in such a way that $\Gamma_{n} \subset \partial S_{n}$.

Let $V$ again be a wandering domain of $g_{b}$ and assume that the free critical value $z_{0}$ is contained in $\bar{V}$. We already know that the set $\left\{\operatorname{Im} g_{b}^{n}(z): z \in V\right\}$ is unbounded, so we can find sequences $\left|n_{k}\right|, m_{k}$ tending monotonously to $\infty$ such that $g_{b}^{m_{k}}(V) \subset S_{n_{k}}$ and $g_{b}^{m}(V) \subset \bigcup_{i=-\left|n_{k}\right|-1}^{\left|n_{k}\right|-1} S_{n_{k}}$ for $m<m_{k}$ (an alternative approach would use a symbolic description). 


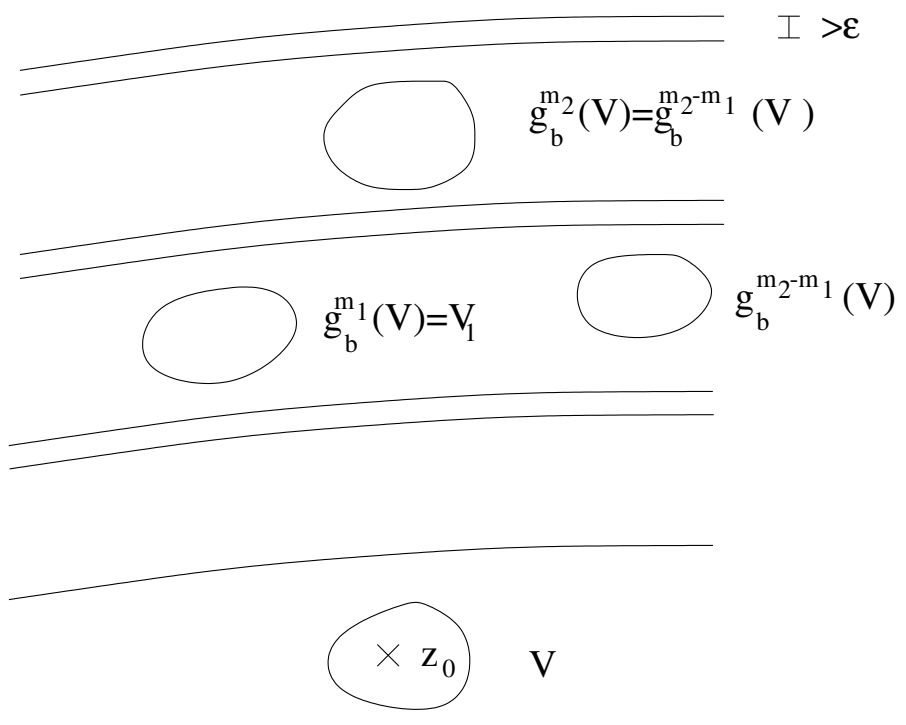

Let $V_{1}=g_{b}^{m_{1}}(V)$, then we have $d\left(g_{b}^{m_{k}-m_{1}}\left(V_{1}\right), S V\left(g_{b}^{m_{k}-m_{1}}\right)\right)>\varepsilon$ for all but finitely many $k$. This is a contradiction to the following lemma.

\section{Lemma 20}

Let $f$ be an entire transcendental function and $U$ be a simply connected Fatou component of $f$. If there exists a constant $C>0$ and a sequence $n_{k}$ tending to $\infty$ such that for any $z \in U, d\left(f^{n_{k}}(z), \partial f^{n_{k}}(U)\right)<C$ holds, then $d\left(f^{n_{k}}(U), S V\left(f^{n_{k}}\right)\right)$ tends to 0 as $k$ tends to $\infty$.

Proof: (Similar ideas have been used in ([7], Lemma 3)

We may assume that $U$ is a wandering domain or Baker domain, otherwise the claim is always true.

Let $U$ and $C$ be as in the hypothesis, $n_{k}$ a subsequence tending to $\infty$ and $z_{0} \in U$ such that $d_{k}:=d\left(f^{n_{k}}\left(z_{0}\right), \partial f^{n_{k}}(U)\right)<C$ and assume that $d\left(f^{n_{k}}(U), S V\left(f^{n_{k}}\right)\right)>\varepsilon$ for all $k \in \mathbb{N}$. The branches $g_{k}=\left(f^{n_{k}}\right)^{-1}$ mapping $f^{n_{k}}\left(z_{0}\right)$ to $z_{0}$ are well-defined and holomorphic on $D\left(z_{n_{k}}, d_{n_{k}}+\varepsilon\right)$. The family given by

$\phi_{k}: \mathbb{D} \rightarrow \mathbb{C}, z \mapsto g_{n_{k}}\left(\left(\varepsilon+d_{n_{k}}\right) z+z_{n_{k}}\right)$ is normal by Montel's Strong Theorem (note that for any function having Baker domains or wandering domains the set of singular values of $f$ has infinitely many elements). Since $\phi_{k}(0)=z_{0}$ for each $k \in \mathbb{N}$, the constant function $\infty$ is not a possible limit function. We have points $v_{k}$ satisfying $v_{k} \in J(f)$ and $\left|z_{k}-v_{k}\right|=d_{k}$. Let $v_{k}^{\prime}=\phi_{k}^{-1}\left(g_{k}\left(v_{k}\right)\right) \in \mathbb{D}$. 
By construction,

$$
\begin{aligned}
& 0<\left|z_{k}-v_{k}\right|=d_{k}<d_{k}+\varepsilon<C+\varepsilon \\
& 0<\left|v_{k}^{\prime}\right|=\left|\frac{v_{k}-z_{k}}{\varepsilon+d_{k}}\right|=\frac{d_{k}}{\varepsilon+d_{k}} \quad<\frac{C}{\varepsilon+C}<1 .
\end{aligned}
$$

and

For any limit function $\phi$ of $\left\{\phi_{k}\right\}_{k \in \mathbb{N}}$ there exists a $v^{\prime}$ such that $\left|v^{\prime}\right|=: r \in(0,1)$ and $\phi\left(v^{\prime}\right) \in J(f)$. This implies that no limit function can be constant. Let $n_{k_{i}}$ be a subsequence of $n_{k}$ such that $\phi_{n_{k_{i}}}$ converges to a holomorphic function $\phi$. Let $D=\phi\left(D\left(v^{\prime},(1-r) / 2\right)\right)$. For all but finitely many $i$ we have $f^{n_{k_{i}}}(D) \subset D\left(z_{n_{k_{i}}}, \varepsilon+d_{n_{k_{i}}}\right) \subset D\left(z_{n_{k_{i}}}, \varepsilon+C\right)$. This is a contradiction to $\phi\left(v^{\prime}\right) \in J(f)$ and the expanding property of the Julia set.

Now we are ready to prove our main results on wandering domains:

\section{Proof of Theorem 3:}

It is known that all limit functions of iterates on a wandering domain are constants contained in the derived set of the postcritical set. In our case this means that limit functions of a wandering domain of $g_{b}$ have to be contained in the set of accumulation points of the forward orbit of the free critical point, denoted with $w\left(z_{0}(b)\right)$.

We already know that $g_{b}$ has no Baker wandering domains, so if a wandering domain exists, then there are infinitely many limit functions of the iterates on this domain. Lemma 19 implies that $\infty \in w\left(z_{0}(b)\right)$.

Suppose that $g_{b}$ has a wandering domain. If $b \in \mathbb{R} \backslash\{0\}$, then symmetry arguments give that $w\left(z_{0}(b)\right) \subset \mathbb{R}$, this is a contradiction to Lemma 19 . If $g_{b}$ has an attracting or parabolic cycle, then the set $w\left(z_{0}(b)\right)$ is finite, so we do not have wandering domains. If $g_{b}$ has a Cremer point or a Siegel disk, we can find a bounded domain $U$ such that $g_{b \mid U}$ is polynomial-like on $U$ and $U$ contains the Siegel disk or the Cremer point, respectively, and the free critical point $z_{0}=z_{0}(b)$. We then have a polynomial $P$ and a quasi-conformal map $\phi$ such that $g_{b \mid U}=\phi^{-1} \circ P \circ \phi$. All critical points of $P$ escape to $\infty$ except for $\phi\left(z_{0}\right)$ which accumulates at the Cremer point or at any point of the boundary of the Siegel disk, respectively. Hence, the forward orbit of $\phi\left(z_{0}\right)$ is contained in $\phi(U)$ and we deduce that $O^{+}\left(z_{0}\right) \in U$. Thus, there are no wandering domains. 


\subsubsection{The parameter plane}

\section{Lemma 21}

For each $m \in \mathbb{C} \backslash\{2\}$ there exists exactly one $b \in \mathbb{C} \backslash\{0\}$ such that $g_{b}$ has a fixed point with multiplier $m$.

\section{Proof:}

We have

$$
\begin{array}{rlr} 
& g_{b}(z) & =z \\
\Rightarrow \quad-1+b z e^{z} & =0 \\
\Rightarrow b z e^{z} & =1 .
\end{array}
$$

Together with $g_{b}^{\prime}(z)=m$ this yields

$$
\begin{array}{rlrl} 
& g_{b}^{\prime}(z) & =m \\
\Rightarrow & 1+b z e^{z}+b e^{z} & =m \\
\Rightarrow & 2+b e^{z} & & =m \\
\Rightarrow b e^{z} & & =m-2 \\
\Rightarrow & \frac{1}{z} & & =m-2 \\
\Rightarrow \quad z & & =(m-2) e^{\frac{1}{2-m}} \\
\Rightarrow b &
\end{array}
$$

\section{Proof of Proposition 5:}

The first part of this proposition follows from the fact that for every $b \in \mathbb{C}^{*}$ the set of critical points moves continuously with respect to the Euclidean metric in some neighborhood of $b$ and that the fundamental domains we constructed can be taken such that they move continuously as well.

Let $U$ be a component of $B\left(\mathcal{F}_{b}\right) \cup\{0\}$. Take for any connected compact set $K$ satisfying $\partial K \subset B\left(\mathcal{F}_{b}\right)$ the compact set $\tilde{K} \supset K$ such that $\partial \tilde{K} \subset K$ and all components of $\tilde{K}$ are simply connected (this is $K$ with "all holes filled"). By Proposition 9.c) we may assume that $0 \notin \tilde{K}$.

Consider continuously moving fundamental domains $D_{b}$ for each $b \in \tilde{K}$. The set $D=\bigcap_{b \in \tilde{K}} D_{b}$ is non-empty, and we can find some $n \in \mathbb{N}$ such that $g_{b}^{n}\left(z_{0}(b)\right) \in D$ for all $b \in \partial \tilde{K}$. Since the map $b \mapsto g_{b}^{n}\left(z_{0}(b)\right)$ is analytic, the maximum principle implies $g_{b}^{n}\left(z_{0}(b)\right) \in D$ for all $b \in \tilde{K}$, so $\tilde{K} \subset B\left(\mathcal{F}_{b}\right)$.

\section{Proof of Theorem 4:}

In section 4.1 .1 we labelled all critical points with $z_{n}(b), n \in \mathbb{Z}$, for parameters in $\mathbb{C} \backslash \mathbb{R}_{0}^{+}$. Here we only need to consider $z_{-1}(b), z_{0}(b)$ and $z_{1}(b)$. The critical points depend holomorphically on the parameter in $\mathbb{C} \backslash \mathbb{R}_{0}^{+}$and we proved that the to the left unbounded rectangle $R_{1}$ given by the points 
$z_{1}(b)-4 / 3$ and Re $z_{1}(b)-4 / 3+i y_{+}(b)$ is invariant, where $y_{+}(b)$ is the solution of the equation

$$
\operatorname{Re} b \sin y+\operatorname{Im} b \cos y=0,
$$

defined by the starting value $y_{+}(-1)=\pi \cdot y_{+}(b)$ depends continuously on $b$ and takes values in $[0,2 \pi)$. Note that $y_{+}(b)+\pi$ is also a solution of this equation, and, since $\operatorname{Im} g_{b}\left(x+i y_{+}(b)\right)>y_{+}(b)$ as calculated above, we obtain $\operatorname{Im} g_{b}\left(x+i\left(y_{+}(b)+\pi\right)\right)<y_{+}(b)+\pi$.

On the other hand, we have $g_{b}\left(z_{1}(b)+1\right)=z_{1}(b)-e$, so $\operatorname{Im} z_{1}(b) \neq y_{+}(b)+\pi$ for all $x \in \mathbb{R}$.

We can check that for some parameter in $\mathbb{R}^{-}$the inequation

Im $z_{1}(b)<y_{+}(b)+\pi$ holds (as done before Corollary 7 ), so this is true for all parameters in $\mathbb{C} \backslash \mathbb{R}_{0}^{+}$by continuity. Hence, the rectangle we constructed is of height less than $\pi$. Note that the equation (4.1) can also be expressed as $\operatorname{Im}\left(b e^{x+i y}\right)=0$. The $\operatorname{strip} S=\left\{z: y_{+}(b)<\operatorname{Im} z<y_{+}(b)+\pi\right\}$ is mapped by the function $z \mapsto b e^{z}$ to the upper or to the lower half plane. Since $z_{1}(b) \in S$ and $\operatorname{Im} b e^{z_{1}(b)}>0$ (Lemma 15), $S$ is mapped to the upper half plane. This means that the rectangle $R_{1}(b)$ is the component of

$\left\{z: b e^{z} \in H^{+}\right\} \cap\left\{z: z-z_{1}(b) \in Q^{--}\right\}$having $z_{1}(b)$ on the boundary, where $Q^{--}$is the lower left quadrant.

Analogously, the rectangle $R_{-1}(b)$ given by $z_{1}(b)-4 / 3$ and

Re $z_{1}(b)-4 / 3+i y_{-}(b)$ is invariant, where $y_{-}(b)$ is the solution of (4.1) defined by the starting value $y_{-}(-1)=\pi$. It is the component of

$\left\{z: \operatorname{Im} b e^{z}<0\right\} \cap\left\{z: z-z_{1}(b) \in Q^{-+}\right\}$having $z_{-1}(b)$ on the boundary, where $Q^{-+}$is the upper left quadrant.

The sets $\overline{R_{1}(b)}$ and $\overline{R_{-1}(b)}$ are non-empty, disjoint, unbounded, contained in the Baker domain $B_{b}$ of $g_{b}$ and move continuously with $b$. Consider the set $A_{1}=\left\{b \in \mathbb{C} \backslash \mathbb{R}_{0}^{+}: g_{b}\left(z_{0}(b)\right) \in \overline{R_{1}(b)}\right\}$.

This is the set

$\left\{b \in \mathbb{C} \backslash \mathbb{R}_{0}^{+}: \operatorname{Im} b e^{z_{0}(b)} \geq 0\right\} \cap\left\{b \in \mathbb{C} \backslash \mathbb{R}_{0}^{+}: g_{b}\left(z_{0}(b)\right)-z_{1}(b) \in \overline{Q^{--}}\right\}$. All components of $A_{1}$ are simply connected and unbounded, all components of $\mathbb{C} \backslash A_{1}$ are simply connected domains.

The same argumentation holds for $A_{-1}=\left\{b \in \mathbb{C} \backslash \mathbb{R}_{0}^{+}: g_{b}\left(z_{0}(b)\right) \in \overline{R_{-1}(b)}\right\}$ as well, so all components of $\mathbb{C} \backslash\left(A_{1} \cup A_{-1}\right)$ are simply connected domains as well. Now, let $M \supset[0,60]$ be a closed, simply connected set contained in $B\left(\mathcal{F}_{b}\right)$ and $\Lambda$ the component of $D(0,60) \backslash\left(A_{1} \cup A_{-1} \cup M\right)$ containing $-2 e^{1 / 2}$, which is the parameter value such that the corresponding function has a super-attracting fixed point. Then $\Lambda$ is a non-empty, bounded and simply connected domain.

Let $V$ be a large square given by points $\pm v, \pm v$ such that $\operatorname{Re} v>0, V$ con- 


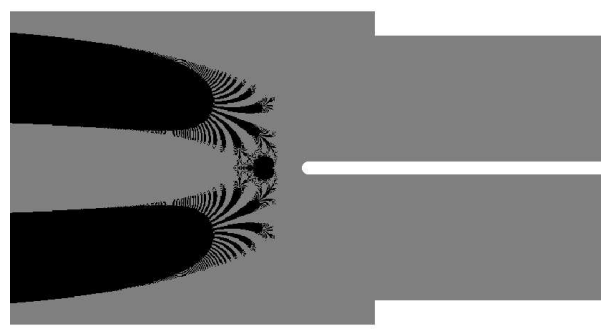

Figure 4.6: Sketch of $\Lambda$

tains $g_{b}\left(z_{0}(b)\right)$ for all $b \in \bar{\Lambda}$ and the images of the segments $L_{1}, L_{2}$ connecting $v, \bar{v}$, and $-v, \overline{-v}$ respectively, do not intersect $V$. Such a $V$ exists as $g_{b}\left(z_{0}(b)\right)$ is analytic in a neighborhood of $\bar{\Lambda}$, and if $v$ is sufficiently large then $L_{1}$ is contained in the set $M_{1}(b)$ for all $b \in \bar{\Lambda}$, so $\operatorname{Re} g_{b}(z)<\operatorname{Re} z$ for all of these points, while points in $L_{2}$ satisfy $\left|g_{b}(|\operatorname{Re} v|+i y)\right|>|v| e^{\operatorname{Re} v} \gg|v|$.

Let $b \in \Lambda$. Let $\Gamma_{1}(b)$ and $\Gamma_{-1}(b)$ as constructed in the proof of Proposition 4 . We know that they separate the plane into two sets with unbounded imaginary parts and a strip $S(b)$. Symmetry arguments give that $z_{0}\left(-2 e^{1 / 2}\right)=-1 / 2$ is contained in $S\left(-2 e^{1 / 2}\right)$. Sinces the curves $\Gamma_{ \pm 1}(b)$ move continuously with $b$ and $z_{0}(b)$ moves holomorphically, the definition of $\Lambda$ gives that $z_{0}(b) \in S(b)$ for all $b \in \Lambda$ (for if $z_{0} \in \partial S(b)$, then $g_{b}\left(z_{0}(b)\right) \in R_{1}(b) \cup R_{-1}(b)$ and $\left.b \notin \Lambda\right)$.

Now, $\partial\left(S(b) \backslash g_{b}^{-1}\left(\gamma_{-1} \cup \gamma_{1}\right)\right)$ is mapped by $g_{b}$ to $\Gamma_{1} \cup \Gamma_{-1}$. Setting $\tilde{S}_{b}=S(b) \backslash g_{b}^{-1}\left(\gamma_{-1} \cup \gamma_{1}\right)$ we note that $g_{b \mid \tilde{S}_{b}}: \tilde{S}_{b} \rightarrow \mathbb{C} \backslash\left(\gamma_{-1} \cup \gamma_{1}\right)$ is a proper map, and since $\tilde{S}_{b}$ contains exactly one critical point we see that it is a $2: 1$ covering.

Hence, there is a component $U_{b}$ of $g_{b}^{-1}\left(V \backslash\left(\overline{R_{1}} \cup \overline{R_{-1}}\right)\right)$ compactly contained in $S_{b}$. Since $g_{b}\left(\partial\left(V \backslash\left(\overline{R_{1}} \cup \overline{R_{-1}}\right)\right)\right) \cap V \backslash\left(\overline{R_{1}} \cup \overline{R_{-1}}\right)=\emptyset$ (here we make use of $g_{b}\left(\overline{R_{i}}\right) \subset R_{i}$ for $\left.i=-1,1\right), U_{b}$ is compactly contained in $U_{b}^{\prime}=V \backslash\left(\overline{R_{1}} \cup \overline{R_{-1}}\right)$. $U_{b}^{\prime}$ contains the critical value, $U_{b}$ the critical point, so the map $g_{b \mid U_{b}}: U_{b} \rightarrow U_{b}^{\prime}$ is a polynomial-like map of degree 2 . By construction, $U_{b}$ and $U_{b}^{\prime}$ move continuously with $b$. 
It only remains to check the hypothesis of the Douady-Hubbard-Theorem. $\Lambda$ is a simply connected domain, we have simply-connected domains $U_{b}$ and $U_{b}^{\prime}$ such that the family $\left\{g_{b \mid U_{b}}: U_{b} \rightarrow U_{b}^{\prime}\right\}_{b \in \Lambda}$ is an analytic family of polynomiallike maps of degree 2 .

Furthermore, if $b \in \partial \Lambda$, then the critical point leaves the domain $U_{b}$ under iteration (note Lemma 26). Since $B\left(\mathcal{F}_{b}\right)$ is open, it is not a problem that $\Lambda$ is open, as we could find a closed subset $\tilde{\Lambda} \subset \Lambda$ such that $\partial \tilde{\Lambda} \subset B\left(F_{b}\right)$ and $\tilde{\Lambda}$ satisfies all for our purpose important properties as well.

So far, the results of Douady and Hubbard give us that $\Lambda$ contains a set $M_{\mathcal{F}}$ which is a covering of the Mandelbrot set, respecting the dynamics. We still have to check the parametric degree of the straightening map (i.e. the continuous map taking $M_{\mathcal{F}}$ into the Mandelbrot set). It is enough to check that the vector $g_{b}\left(z_{0}(b)\right)-z_{0}(b)$ turns once around 0 as $b$ describes the boundary of $\Lambda$. We have $g_{b}\left(z_{0}(b)\right)-z_{0}(b)=z_{0}(b)-2+\frac{1}{z_{0}(b)}-z_{0}(b)=-2+\frac{1}{z_{0}(b)}$, and since the function mapping $b$ to $z_{0}(b)$ is univalent in $\mathbb{C} \backslash \mathbb{R}_{0}^{+} \supset \Lambda$, the winding number we are looking for is equal to one.

\section{Lemma 22}

a)

If $g_{b}^{\prime}\left(z_{0}\right)=0$ and $\operatorname{Im} z_{0}>0$, then $\operatorname{Im} g_{b}\left(z_{0}\right)<\operatorname{Im} g_{b}\left(x+i \operatorname{Im} z_{0}\right)<\operatorname{Im} z_{0}$ and $\frac{d \operatorname{Im} g_{b}\left(x+i \operatorname{Im} z_{0}\right)}{d x}<0$ for all $x<R e z$. The analogous statement is true if Im $z_{0}<0$. If $z_{0} \in \mathbb{R}$, then $\mathbb{R}$ is invariant.

b)

If $g_{b}^{\prime}\left(z_{0}\right)=0$ and

1. the line $\left\{x+i \operatorname{Im} z_{0}: x<\right.$ Re $\left.z_{0}\right\}$ is contained in $M_{c}$ or

2. $x_{0}<-1$,

c)

then $\operatorname{Re} g_{b}\left(x+i \operatorname{Im} z_{0}\right)<\operatorname{Re} g_{b}\left(z_{0}\right)$ for all $x<\operatorname{Re} z_{0}$.

If $g_{b}^{\prime}\left(z_{0}\right)=0$ and $x_{0}>-1$, then Re $g_{b}\left(x+i \operatorname{Im} z_{0}\right)<\operatorname{Re} g_{b}\left(z_{0}\right)$ for all $x \in\left[-2, \operatorname{Re} z_{0}\right]$. 


\section{Proof:}

Let $b=c+i d$ and $z_{0}=x_{0}+i y_{0}$.

a)

Let

$$
\begin{aligned}
h(x) & =\operatorname{Im} g_{b}\left(x+i y_{0}\right)-y_{0} \\
& =\left(x\left(c \sin y_{0}+d \cos y_{0}\right)+y_{0}\left(c \cos y_{0}-d \sin y_{0}\right)\right) e^{x} .
\end{aligned}
$$

$h^{\prime}(x)=\left((x+1)\left(c \sin y_{0}+d \cos y_{0}\right)+y_{0}\left(c \cos y_{0}-d \sin y_{0}\right)\right) e^{x}$ has exactly one root, it has to be $x_{0}$. Now $h(x) \rightarrow 0$ as $x$ tends to $-\infty$ and $h\left(x_{0}\right)<0$ (Lemma 15d)) implies a).

b)

If the line $L=\left\{x+i y_{0}: x<x_{0}\right\}$ is contained in $M_{c}$, then $\operatorname{Re} g_{b}^{\prime}(z) \in(0,2)$, this implies the claim.

If $x_{0}<-1$, we calculate

$$
\begin{aligned}
g_{b}^{\prime}(x+i y)= & 1+(c((x+1) \cos y-y \sin y)+d(-y \cos y-(x+1) \sin y)) e^{x}+ \\
& +i(c((x+1) \sin y+y \cos y)+d(-y \sin y+(x+1) \cos y)) e^{x} \\
=: & 1+\left((x+1) c_{1}-c_{2}\right) e^{x}+i\left((x+1) c_{2}+c_{1}\right) e^{x}
\end{aligned}
$$

Assume that $c_{1}$ is negative, then $\operatorname{Im} g_{b}^{\prime}\left(x_{0}+i y_{0}\right)=0$ implies $c_{2}<0$ and $\operatorname{Re} g_{b}\left(z_{0}\right)>1$; hence, this is impossible, so $c_{1} \geq 0$. This yields

$\operatorname{Re} g_{b}\left(x+i y_{0}\right)=x-1+\left(c_{1} x-c_{2}\right) e^{x}<x_{0}-1+\left(c_{1} x_{0}-c_{2}\right) e^{x_{0}}=\operatorname{Re} g_{b}\left(z_{0}\right)$ for $x<x_{0}$.

c)

Again we use the notation

$g_{b}^{\prime}(x+i y)=1+\left((x+1) c_{1}-c_{2}\right) e^{x}+i\left((x+1) c_{2}+c_{1}\right) e^{x}$ as in b). Let $x_{0}>-1$ and assume that $c_{1}$ is positive. Then $\operatorname{Im} g_{b}^{\prime}\left(x_{0}+i y_{0}\right)=0$ implies $c_{2}<0$ and $\operatorname{Re} g_{b}^{\prime}\left(z_{0}\right)>1$; again, this is impossible, so $c_{1} \leq 0$. This gives $c_{2}>0$ and $\operatorname{Re} g_{b}^{\prime \prime}(x+i y)=\left((x+2) c_{1}-c_{2}\right) e^{x}<0$ on $\left[-2, x_{0}\right)$, so $\operatorname{Re} g_{b}^{\prime}\left(x+i y_{0}\right)>\operatorname{Re} g_{b}^{\prime}\left(x+i y_{0}\right)=0$ on $\left[-2, x_{0}\right)$, proving our claim.

\section{Lemma 23}

If $\operatorname{Im} b \geq 0,|\operatorname{Im} z|>\frac{2}{3}$ and $g_{b}^{\prime}(z)=0$, then the to the left unbounded rectangle given by $z-\frac{4}{3}$ and $\bar{z}-\frac{4}{3}$ is contained in $M_{1} \cap M_{c}$.

\section{Proof:}

Let $z=x+i y$. By Corollary 5 and Lemma 14 we know that $R \subset M_{c}$ and that if suffices to prove that $z_{n}-\frac{4}{3} \in M_{1}$. Again using the above-mentioned 
lemma we know that $y^{2}=\frac{e^{-2 x}}{\left|b^{2}\right|}-(x+1)^{2}=: h(x)$ and $-2\left(\frac{e^{-2 x}}{\mid b^{2}}+x+1\right)=h^{\prime}(x)<0$. We have

$$
\begin{aligned}
\frac{e^{-2\left(x-\frac{4}{3}\right)}}{\left|b^{2}\right|}-\left(x-\frac{4}{3}\right)^{2} & =e^{\frac{8}{3}} \frac{e^{-2 x}}{\left|b^{2}\right|}-x^{2}+\frac{8}{3} x-\frac{16}{9} \\
& =e^{\frac{8}{3}}\left(y^{2}+(x+1)^{2}\right)-x^{2}+\frac{8}{3} x-\frac{16}{9} \\
& =e^{\frac{8}{3}} y^{2}+e^{\frac{8}{3}}(x+1)^{2}-x^{2}+\frac{8}{3} x-\frac{16}{9} \\
& =: e^{\frac{8}{3}} y^{2}+P(x) .
\end{aligned}
$$

The polynomial $P$ has a minimum at $t=\frac{-e^{\frac{8}{3}}-\frac{4}{3}}{e^{\frac{8}{3}}-1}$, and the minimum value is larger than $-\left(e^{\frac{8}{3}}-1\right) \frac{4}{9}$. Hence,

$\frac{e^{-2\left(x-\frac{4}{3}\right)}}{\left|b^{2}\right|}-\left(x-\frac{4}{3}\right)^{2}-y^{2}=\left(e^{\frac{8}{3}}-1\right) y^{2}+P(x)>\left(e^{\frac{8}{3}}-1\right) \frac{4}{9}-\left(e^{\frac{8}{3}}-1\right) \frac{4}{9}=0$.

\section{Lemma 24}

If Im $b \geq 0, \operatorname{Im} z \in\left(-\frac{\pi}{2}, 0\right)$ and $g_{b}^{\prime}(z)=0$, then Re $z<-1$ and Re $g_{b}(z)<\operatorname{Re} z-2$.

\section{Proof:}

Let $b=c+i d$. Due to symmetry arguments we may restrict to $d \geq 0$ and $\operatorname{Im} y>0$.

$$
\begin{aligned}
g_{b}^{\prime}(x+i y)= & 1+b(z+1) e^{z} \\
= & 1+((c(x+1)-d y) \cos y-(d(x+1)+c y) \sin y) e^{x}+ \\
& +i((c(x+1)-d y) \sin y+(d(x+1)+c y) \cos y) e^{x} \\
= & 1+(c((x+1) \cos y-y \sin y)+d(-y \cos y-(x+1) \sin y)) e^{x} \\
& +i(c((x+1) \sin y+y \cos y)+d(-y \sin y+(x+1) \cos y)) e^{x} \\
=: & 1+\left(c a_{1}-d a_{2}\right) e^{x}+i\left(c a_{2}+d a_{1}\right) e^{x} .
\end{aligned}
$$

Assume that $g_{b}^{\prime}(x+i y)=0, y \in\left(-\frac{\pi}{2}, 0\right)$ and $x>-1$. We have $d a_{2}<0$. If $c a_{1} \neq 0$, then $\operatorname{Im} g_{b}^{\prime}(x+i y)=0$ implies $c a_{1}>0$ and $\operatorname{Re} g_{b}^{\prime}(x+i y)>1$, and if $c a_{1}=0$, then we have $\operatorname{Re} g_{b}^{\prime}(x+i y)>1$ as well, so $g_{b}^{\prime}(x+i y) \neq 0$ in both cases. The last statement is a consequence of Lemma 15.

\section{Lemma 25}

There exists $a b \in \mathbb{R}^{-}$such that $\operatorname{Im} z_{1}(b)>\pi$ and $\operatorname{Im} z_{-2}(b)<-2 \pi$.

\section{Proof:}

First, we show that the strip $\{0<\operatorname{Im} z \leq \pi\}$ does not contain critical points 
if $b \in \mathbb{R}^{-}$. We have

$g_{b}^{\prime}(x+i y)=1+b((x+1) \cos y-y \sin y) e^{x}+i b((x+1) \sin y+y \cos y) e^{x}$. If $y>0$ and $\sin y \geq 0$, then $\operatorname{Im} g_{b}^{\prime}(x+i y)=0$ implies $\cos y(x+1) \leq 0$ yielding $\operatorname{Re} g_{b}^{\prime}(x+i y) \geq 1$.

For symmetry reasons the strip $\{0>\operatorname{Im} z \geq-\pi\}$ does not contain critical points neither, so $\operatorname{Im} z_{-1}(b)<-\pi$. If $|b|$ is sufficiently small, then the curve $\gamma=\partial M_{c} \cap\{z:-\pi \geq \operatorname{Im} z \geq-2 \pi\}$ is close to a segment of length $\pi$, in particular, its length is less than $\frac{3 \pi}{2}$. Furthermore, again for $|b|$ sufficiently small we have $\gamma \subset\{z: \operatorname{Re} z>-2\}$, so $g_{b}^{\prime \prime}(z)=b(z+2) e^{z} \neq 0$ on $\gamma$ and using our definition of $M_{c}$ we obtain

$$
\begin{aligned}
\left|g_{b}^{\prime \prime}(z)\right| & =\left|b(z+1) e^{z}+b e^{z}\right| \\
& \leq\left|b(z+1) e^{z}\right|+\left|b e^{z}\right| \\
& =\left|b(z+1) e^{z}\right|+\left|\frac{1}{z+1}\right| \\
& =1+\left|\frac{1}{z+1}\right| \\
& \leq 1+\frac{1}{\pi} \\
& <\frac{4}{3}
\end{aligned}
$$

for $z \in \gamma$. Since $g_{b}^{\prime}(\gamma) \subset \partial D(1,1)$, this implies that there is at most one critical point contained in $\gamma$, which is the point $z_{-1}(b)$.

Hence, $\operatorname{Im} z_{-2}<-2 \pi$.

\section{Lemma 26}

If $b \in \partial D(0,60) \backslash\{60\}$, then $z_{0}(b)$ leaves any compact set contained in $\{z:|\operatorname{Im} z|<4 \pi\}$ under iteration.

\section{Proof:}

Let $S_{4 \pi}:=\{z:|\operatorname{Im} z|<4 \pi\}$ and $|b|=60$, but $b \neq 60$. Then $\frac{e^{-2(-1.03)}}{|b|^{2}}-(-1.03+1)^{2}<0$ and $\frac{e^{-2(-1)}}{|b|^{2}}-(-1+1)^{2}>0$, so the boundary of $M_{c}$ consists of two components. Take a simply connected curve $\gamma$ enclosing the bounded one but not intersecting the other one. For every $z \in \gamma$ we have $1<\left|b(z+1) e^{z}\right|=\left|g_{b}^{\prime}(z)\right|$. Rouché's Theorem gives that the number of critical points of $g_{b}$ in the interior of $\gamma$ is equal to the number of roots of $b(z+1) e^{z}$ in the same domain, this number is one. For $b_{0}=-60$ the critical point is $z_{0}(-60)$, by continuity it is always the point $z_{0}(b)$ for $b \in \partial D(0,60) \backslash\{60\}$. Let $z_{0}=z_{0}(b)=x_{0}+i y_{0}$. Our calculations above imply that $x_{0}>-1.03$, 
and Lemma 14 gives $\left|y_{0}\right|<\frac{1}{2}$, so

$$
\begin{aligned}
\left|g_{b}\left(z_{0}\right)\right| & =\left|z_{0}-2-b e^{z_{0}}\right| \\
& >60 e^{x_{0}}-\left(\frac{1}{4}+x_{0}^{2}\right)^{\frac{1}{2}} \\
& >60 e^{x_{0}}-4 \\
& \geq 60 e^{-1.03}-4 \\
& >\left(2 \ln 60+16 \pi^{2}\right)^{\frac{1}{2}}
\end{aligned}
$$

If $g_{b}\left(z_{0}\right) \in S_{4 \pi}$, then $\left|\operatorname{Re} g_{b}\left(z_{0}\right)\right|>2 \ln 60$. Now $\frac{1}{4} \frac{e^{-2(-2 \ln 60)}}{3600}-(2 \ln 60)^{2}=\frac{1}{4} 3600-4(\ln 60)^{2}>(4 \pi)^{2}$, so $h(x)=\frac{1}{4} \frac{e^{-2 x}}{3600}-x^{2}>(4 \pi)^{2}$ for all $x<-2 \ln 60 .\left|h^{\prime}(x)\right|=2\left(\frac{e^{-2 x}}{3600}+x\right)>1$ for all $x<-2 \ln 60$, and our results in section 3.1 .1 give that the domain $D_{0.5,2 \ln 60,0.5}$ is a fundamental domain of $B_{b}$ containing the set $\{z: \operatorname{Re} z<-2 \ln 60,|\operatorname{Im} z|<4 \pi\}$.

Hence, if $g_{b}\left(z_{0}\right) \in S$ and $\operatorname{Re} g_{b}\left(z_{0}\right)<-2 \ln 60$, then $g_{b}\left(z_{0}\right) \in B_{b}$ and $g_{b}^{n}\left(z_{0}\right) \rightarrow \infty$.

The only remaining possibility is $\operatorname{Re} g_{b}\left(z_{0}\right)>2 \ln 60$, but in this case we set $z_{1}=g_{b}\left(z_{0}\right)$ and obtain $\left|z_{2}\right|=\left|g_{b}\left(z_{1}\right)\right|=\left|z_{1}-1+b z_{1} e^{z_{1}}\right| \geq\left|b e^{z_{1}}-1\right|\left|z_{1}\right|-1>$ $60\left|z_{1}\right| . \quad z_{3}=g_{b}\left(z_{2}\right)$ is not contained in the strip $S_{4 \pi}$ or is contained in the fundamental domain $D_{b,-2 \ln 60,0.5}$ of $B_{b}$ or satisfies $\left|z_{3}\right|>60\left|z_{2}\right|$, by repeating this process we obtain that $g_{b}\left(z_{0}\right)$ leaves any compact set contained in $S_{4 \pi}$ under iteration.

\section{Lemma 27}

There are continuous functions $y_{+}: \overline{H^{+}} \backslash\{0\} \rightarrow[\pi, 2 \pi]$ and $y_{-}: \overline{H^{+}} \backslash\{0\} \rightarrow[-\pi, 0]$ such that for any $n \geq 0, x \in \mathbb{R}$ and $b \in H^{+} \cup \mathbb{R}^{-}$ the following holds:

1. $\operatorname{Im} g_{b}\left(x+i\left(y_{+}(b)+2 \pi n\right)\right)>y_{+}(b)+2 \pi n$

2. $\operatorname{Im} g_{b}\left(x+i\left(y_{+}(b)+2 \pi n+\pi\right)\right)<y_{+}(b)+2 \pi n+\pi$

3. $\operatorname{Im} g_{b}\left(x+i\left(y_{-}(b)-2 \pi n\right)\right)<y_{-}(b)-2 \pi n$

4. $\operatorname{Im} g_{b}\left(x+i\left(y_{-}(b)-2 \pi n-\pi\right)\right)<y_{-}(b)-2 \pi n-\pi$

5. $y_{+}(b)=\pi$ and $y_{-}(b)=-\pi$ for $b \in \mathbb{R}^{-}$

6. $\frac{d \operatorname{Im} g_{b}\left(x+i\left(y_{+}(b)+2 \pi n\right)\right)}{d x}>0$ and $\frac{d \operatorname{Im} g_{b}\left(x+i\left(y_{-}(b)+2 \pi n\right)\right)}{d x}<0$ for all $x \in \mathbb{R}$. 


\section{Proof:}

Let $b=c+i d$. For $b_{0} \in \mathbb{R}^{-}, y_{+}\left(b_{0}\right)=\pi$ is a solution of the equation $c \sin y+d \cos y=0$, and the solution of this equation depends continuously on $b$ as $b \neq 0$ is contained in the closed upper half plane, so this gives rise to a continuous function $y_{+}: \overline{H^{+}} \backslash\{0\} \rightarrow \mathbb{R}$. For points $b$ in the upper half plane $H^{+}$close to $\mathbb{R}^{-}$we have $c<0, d>0$, and $y_{+}(b)$ is contained in the interval $(\pi, 2 \pi)$. Furthermore, if $b \in H^{+}$, then $\pi$ and $2 \pi$ are no solutions of the equation mentioned above, so $y_{+}$takes values in $[\pi, 2 \pi]$ only.

For all $x \in \mathbb{R}, n \in \mathbb{N}$ and $d>0$ we have

$$
\begin{aligned}
& \operatorname{Im} g_{b}\left(x+i\left(y_{+}(b)+2 \pi n\right)\right) \\
= & y_{+}(b)+2 \pi n+\left(x\left(c \sin \left(y_{+}(b)+2 \pi n\right)+d \cos \left(y_{+}(b)+2 \pi n\right)\right)+\right. \\
& \left.+\left(y_{+}(b)+2 \pi n\right)\left(-d \sin \left(y_{+}(b)+2 \pi n\right)+c \cos \left(y_{+}(b)+2 \pi n\right)\right)\right) e^{x} \\
= & y_{+}(b)+2 \pi n-\left(y_{+}(b)+2 \pi n\right)\left(-d \sin \left(y_{+}(b)+2 \pi n\right)+c \cos \left(y_{+}(b)+2 \pi n\right)\right) e^{x} \\
= & y_{+}(b)+2 \pi n-\frac{y_{+}(b)+2 \pi n}{d}\left(c^{2}+d^{2}\right) \sin \left(y_{+}(b)+2 \pi n\right) e^{x} \\
> & y_{+}(b)+2 \pi n,
\end{aligned}
$$

for $b \in \mathbb{R}^{-}$we have $\operatorname{Im} g_{b}\left(x+i\left(y_{+}(b)+2 \pi n\right)\right)>y_{+}(b)+2 \pi n$ as well.

From this calculations one can also see the second statement, since we only have to change the sign of $\sin \left(y_{+}(b)+2 \pi n\right)$, which gives the desired result. Analogously, we consider the function $y_{-}: \overline{H^{+}} \backslash\{0\} \rightarrow \mathbb{R}$ defined by the equation $c \sin y+d \cos y=0$ and the value $y_{-}(b)=-\pi$ for $b \in \mathbb{R}^{-}$. For $b \in H^{+}$close to $\mathbb{R}^{-}$we have $y_{-}(b) \in(-\pi, 0)$, while $y_{-}(b) \neq-\pi$ for $b \in H^{+}$, so $y_{-}$takes values in $(-\pi, 0)$ only.

For all $x \in \mathbb{R}, d>0$ we have $\operatorname{Im} g_{b}\left(x+i y_{-}(b)-2 \pi n\right)=$ $=y_{-}(b)-2 \pi n-\frac{y_{-}(b)-2 \pi n}{d}\left(c^{2}+d^{2}\right) \sin \left(y_{-}(b)-2 \pi n\right) e^{x}<y_{-}(b)$, for $b \in \mathbb{R}^{-}$ this holds as well. Again, statement 4 can be seen from the same calculation. 5. follows immediately from the definitions, and to see 6 . we use for $b \notin \mathbb{R}^{-}$ the already calculated formula

$\operatorname{Im} g_{b}\left(x+i\left(y_{+}(b)+2 \pi n\right)\right)=y_{+}(b)+2 \pi n-\frac{y_{+}(b)+2 \pi n}{d}\left(c^{2}+d^{2}\right) \sin \left(y_{+}(b)+2 \pi n\right) e^{x}$ giving the desired result, for $b \in \mathbb{R}^{-}$the calculations are analogous.

\subsubsection{A related family}

\section{Proof of Theorem 5:}

As mentioned in the text, we have conjugacies between the functions $f_{a}$ and $g_{b}$ if $b=-a e^{\frac{1}{a}}$. We also have seen that if $b \in \mathbb{R}^{+}$, then all critical points 
are contained in the Baker domain of $g_{b}$. Now we examine the preimages of the positive real axis with respect to the function $h(z)=-z e^{\frac{1}{z}}$, for all parameters $a$ contained in these preimages the Baker domain of $f_{a}$ contains all critical points of $f_{a}$.

0 is an essential singularity of $h$, and $\mathbb{R}^{+}$does not contain a singular value. We see that the preimages of $\mathbb{R}^{+}$separate the complex plane into infinitely many domains, only one of them is unbounded.

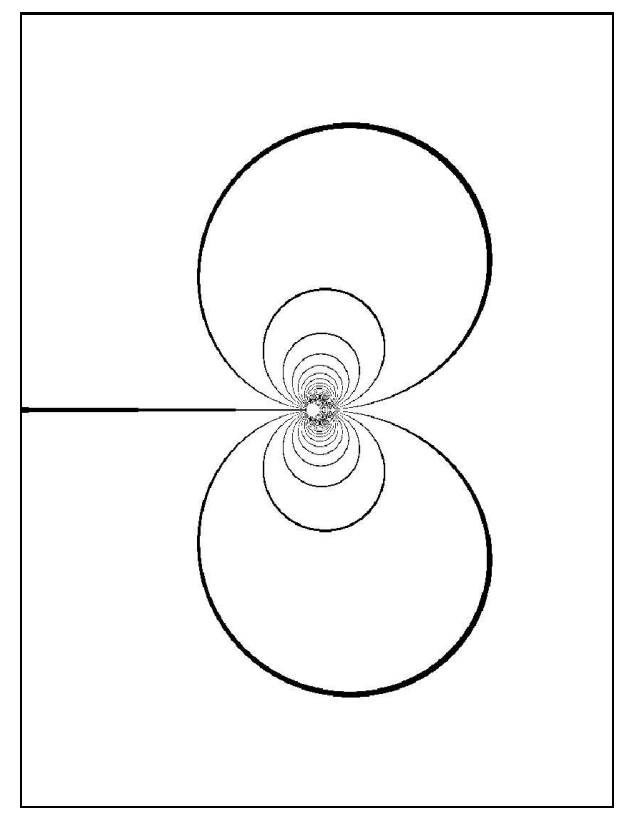

Figure 4.7: Neighborhood of $h^{-1}\left(\mathbb{R}^{+}\right)$

Each bounded one is mapped 1:1 onto $\mathbb{C} \backslash \mathbb{R}_{0}^{+}$, the unbounded one is mapped $2: 1$ onto the same set. The only critical point of $h$ is 1 (obviously contained in the unbounded component of $\mathbb{C} \backslash h^{-1}(\mathbb{R})$ ), the critical value $-e$ is exactly the "mouth" of the cardiod of the Mandelbrot copy of $\mathcal{F}_{b}$. This last fact gives that there is a 2:1-covering of the Mandelbrot set in the parameter plane of $\mathcal{F}_{a}$, and since 0 is an essential singularity of $h$, we have a sequence of preimages of the Mandelbrot copy of $\mathcal{F}_{b}$ tending to $\{0\}$, and all of these copies are Mandelbrot copies for $\mathcal{F}_{a}$. 


\section{Proof of Lemma 4:}

The critical points of $f_{0}$ are the points $z_{k}=(2 k+1) \pi i, k \in \mathbb{Z}$. If $\varepsilon>0$, then $f_{\varepsilon}^{\prime}\left(-1+\frac{1}{\varepsilon}\right)=1>0$, while $f_{\varepsilon}^{\prime}(x)<0$ for sufficiently large $x \in \mathbb{R}^{+}$. Hence, there exists a critical point of $f_{\varepsilon}$ in $\left(-1+\frac{1}{\varepsilon}, \infty\right)$.

\subsection{Generalization}

The function $f(z)=z-c+P(z) e^{a z}$ is conformally conjugate to the function $g(z)=z-a c+a P\left(\frac{z}{a}\right) e^{z}$. Hence, we may assume in our proofs that $a=1$. Since we proved some similar statements before, we will concentrate on the differences to the special case if we do not need new methods.

\section{Proof of Lemma 6:}

Assume that $f$ has an asymptotic value. Then 0 is an asymptotic value of the function $z \mapsto \frac{f(z)}{z}=1-\frac{c}{z}+\frac{P(z)}{z} e^{a z}$, so -1 is an asymptotic value of $g(z)=\frac{P(z)}{z} e^{a z}$. But $g$ has only one asymptotic value, namely 0 .

\section{Proof of Lemma 5:}

If $P \equiv b \in \mathbb{C}^{*}$, then $f$ is a logarithmic lift of $g(z)=e^{-c} z e^{b z}$. If $P$ is not constant, then the set of critical points is not $2 \pi i$-periodic, as we will see below. Hence, it cannot be a logarithmic lift.

\section{Proof of Theorem 6:}

Fix a $\delta \in(0, \operatorname{Re} c)$ and consider the set

$$
\begin{aligned}
M_{\delta} & =\{z:|f(z)-(z-c)|<\delta\} \\
& =\left\{z:\left|P(z) e^{z}\right|<\delta\right\} .
\end{aligned}
$$

The set $\left\{\operatorname{Re} z: z \in M_{\delta}\right\}$ is bounded above, since the right-hand side of the equation $|P(z)|=\delta e^{-\operatorname{Re} z}$ tends to 0 as $\operatorname{Re} z$ tends to $+\infty$, while the lefthand side tends to $\infty$.

If $d=\operatorname{deg} P=0$, then $M_{\delta}$ is just a half plane. If $d=\operatorname{deg} P \geq 1$, then we see from the calculation

$$
\begin{array}{ll}
|P(z)|^{2} & =\delta^{2} e^{-2 \operatorname{Re} z} \\
\Leftrightarrow \quad \sum_{i+j \leq d} a_{i, j} x y^{j} & =\delta^{2} e^{-2 x}
\end{array}
$$

that the left-hand side is dominated by the addend $a_{0,2 d} y^{2 d}$, we conclude that there is a half plane $H_{R}=\{z: \operatorname{Re} z<-R\}$ such that the set $H_{R} \cap M_{\delta}$ is 
the union of the graphs of two monotonuos functions and $x+i y \in H_{R} \cap M_{\delta} \Rightarrow|y|^{2 d} \approx \delta^{2} \frac{e^{-2 x}}{\mid a_{0,2 d}}$.

As in section 4.1 .1 we can take an $R>0$ such that the set $D_{\delta, R}=H_{R} \cap M_{\delta}$ is forward invariant, and the same arguments give that it is an absorbing domain and the last statement of the theorem.

\section{Proof of Proposition 6:}

All critical points are contained in the set $\gamma_{c}=\left\{z:\left|f^{\prime}(z)-1\right|=1\right\}=\left\{z:\left|\left(P(z)+P^{\prime}(z)\right) e^{z}\right|=1\right\}$. Lemma 29 gives that $\gamma_{c}$ and $\partial M_{\delta}$ (see the proof of Theorem 6) are asymptotically parallel with a distance of $\ln \delta$. For any critical point $z$ of $f$ we have

$$
\begin{aligned}
& f^{\prime}(z) & = \\
\Rightarrow 1+\left(P(z)+P^{\prime}(z)\right) e^{z} & = & 0 \\
\Rightarrow P(z) e^{z} & & -1-P^{\prime}(z) e^{z} \\
\Rightarrow f(z) & = & z-1-c-P^{\prime}(z) e^{z}
\end{aligned}
$$

Now $P^{\prime}(z) e^{z}$ tends to 0 as $z \in \gamma_{c}$ and $|z| \rightarrow \infty$. Hence, for any $\varepsilon>0$ we have $f(z) \in D(z-1-c, \varepsilon)$ for all but finitely many critical points of $f$. If $|\ln \operatorname{Re} c|<\operatorname{Re} 1+c$, then we can find an $\delta>0$ such that $\ln \operatorname{Re} c<\operatorname{Re} 1+c+\delta$ and all but finitely many critical points of $f$ are mapped into an absorbing domain $D_{\delta, R}$ as constructed above. Therefore, all but finitely many critical values are contained in the Baker domain $B$. The condition $|\ln \operatorname{Re} c|<\operatorname{Re} 1+c$ is satisfied for all $c>0.3$.

\section{Proof of Lemma 7:}

For any constants $R>0, \varepsilon>0$ we can find a bounded domain $U_{R, \varepsilon}$ such that $|P(z+v)|<(1+\varepsilon)|P(z)|$ for all $z \in U_{R, \varepsilon}$ and $v \in D(0, R)$. Let $\delta=\operatorname{Re} c / 3, U=U_{\frac{4}{3} c, \exp (\delta)-1}$ and $D_{\delta}$ be a absorbing domain for $f$ as constructed in section 3.2 . There exists a translation $D$ of $D_{\delta}$ to the left such that $D \cap U=\emptyset . \quad D$ is an absorbing domain as well and satisfies $z \in D \Rightarrow\left|P(z) e^{z}\right|<\delta$. Let $h(z)=z-c$. For any compact set $K \subset D$ we have

$$
K \subset\{z: \operatorname{Re} z<x\} \Rightarrow f(K) \subset\{z: \operatorname{Re} z<x-\operatorname{Re} c+\delta\}
$$

This yields

$$
\max _{z \in f(K)}\left|P(z) e^{z}\right|=\max _{z \in K}\left|P(f(z)) e^{f(z)}\right| \leq \max _{z \in K}\left|e^{\delta} P(z) e^{z-\operatorname{Re} c+\delta}\right|=e^{-\delta} \max _{z \in K}\left|P(z) e^{z}\right| .
$$


By induction we obtain

$$
\begin{aligned}
d\left(f^{n}(K), h^{n}(K)\right) & \leq \sum_{n=1}^{m} \max _{z \in f^{n-1}(K)}\left|P(z) e^{z}\right| \\
& \leq \sum_{n=1}^{m} \max _{z \in K}\left|P\left(f^{n-1}(z)\right) e^{f^{n-1}(z)}\right|<\delta \sum_{n=1}^{\infty}\left(e^{-\delta}\right)^{n}<\infty
\end{aligned}
$$

\section{Lemma 28}

If $R e$ ac $>\ln 2$, then there exist $\varepsilon>0, R>0$ such that the set $f\left(\left\{z:|z|>R,\left|f^{\prime}(z)\right|<1+\varepsilon\right\}\right)$ is contained in the Baker domain $B$ of $f$.

\section{Proof:}

Let $\varepsilon \in(0, \operatorname{Re} c)$. We can find an $R=R(\varepsilon)>0$ such that $\left|\left(P(z)+P^{\prime}(z)\right) e^{z}\right|<2+\varepsilon$ and $|z|>R$ imply $\left|P^{\prime}(z) e^{z}\right|<\varepsilon$ (since the degree of $P^{\prime}$ is smaller than the degree of $P$ ).

If $\left|f^{\prime}(z)\right|<1+\varepsilon$, then $\left|1+\left(P(z)+P^{\prime}(z)\right) e^{z}\right|<1+\varepsilon$, so $\left|\left(P(z)+P^{\prime}(z)\right) e^{z}\right|<2+\varepsilon$. Hence, $z$ is contained in the set $M_{\varepsilon}=\left\{z:\left|\left(P(z)+P^{\prime}(z)\right) e^{z}\right|<2+\varepsilon\right\}$ and we choose an absorbing domain $D=D_{e^{-\varepsilon}, \tilde{R}}$ as constructed above with $\tilde{R}>R$. The asymptotic horizontal distance between $\partial M_{\varepsilon}$ and $\partial D$ in the sense of Lemma 29 is $\ln (2+\varepsilon)-\ln e^{-\varepsilon}=\ln (2+\varepsilon)+\varepsilon$. If $|z|>\tilde{R}$, then we obtain

$$
\begin{aligned}
f(z) & =z-c+P(z) e^{z} \\
& =z-1-c+1+P(z) e^{z}+P^{\prime}(z) e^{z}-P^{\prime}(z) e^{z} \\
& =z-1-c+f^{\prime}(z)-P^{\prime}(z) e^{z} \\
& \in D(z-1-c, 1+2 \varepsilon) .
\end{aligned}
$$

If we choose $\varepsilon$ sufficiently small, then

$$
\begin{array}{rlrl} 
& \operatorname{Re} c & >\ln (2+\varepsilon)+3 \varepsilon \\
\Rightarrow \operatorname{Re} c-2 \varepsilon & >\ln (2+\varepsilon)+\varepsilon \\
\Rightarrow|-1-\operatorname{Re} c+1+2 \varepsilon| & >\ln (2+\varepsilon)+\varepsilon .
\end{array}
$$

By increasing $\tilde{R}$ if necessary we obtain our claim. 


\section{Lemma 29}

Let $P$ be a polynomial of degree $d, Q$ a polynomial of degree $d^{\prime}<d$, $a<b \in \mathbb{R}^{+}$and $\gamma_{1}, \gamma_{2}$ be the curves in $\mathbb{C}$ defined by the equations $\left|P(z) e^{z}\right|=a$ and $\left|(P(z)+Q(z)) e^{z}\right|=b$, respectively. Then the "asymptotic horizontal distance" between $\gamma_{1}$ and $\gamma_{2}$ tends to $\ln b-\ln a$ in the sense that for each $\varepsilon>0$ we can find an $y_{0}>0$ such that $|y|>y_{0}, x_{1}+i y \in \gamma_{1}$ and $x_{2}+i y \in \gamma_{2}$ imply $\left|\left(x_{2}-x_{1}\right)-(\ln b-\ln a)\right|<\varepsilon$.

\section{Proof:}

As we have seen above, there exists an $R>0$ such that the set $M_{a}=\left\{z:\left|P(z) e^{z}\right|=a,|z|>R\right\}$ consists of two disjoint curves, each of them is the graph of a monotonous function and is contained in a half plane $\left\{z: \operatorname{Re} z<z_{0}\right\}$. Since $\operatorname{deg} Q<\operatorname{deg} P$, we have $\left|Q(z) e^{z}\right| \rightarrow 0$ as $z \in \partial M_{a}$ and $|z| \rightarrow \infty$. Furthermore, for any $\varepsilon>0, K>0$ there exists a $C>0$ such that $|P(z+K)|>|P(z)| e^{-\varepsilon / 2}$ for all $|z|>C$.

Let $\varepsilon>0, \delta=\ln b-\ln a$ and $y_{0}$ such that $\left|Q(z) e^{z}\right|<b\left(e^{\varepsilon / 2}-1\right)$ for all $z \in \partial M_{a}$ satisfying $|\operatorname{Im} z|>y_{0}$ and such that $|P(z+\delta+\varepsilon)|>|P(z)| e^{-\varepsilon / 2}$ for all $z \notin D\left(0, y_{0}\right)$. Let $x_{1}+i y$ be as in the hypothesis.

Then

$$
\begin{aligned}
& \left|\left(P\left(x_{1}+i y+\delta+\varepsilon\right)+Q\left(x_{1}+i y+\delta+\varepsilon\right)\right) e^{x_{1}+i y+\delta+\varepsilon}\right| \\
\geq & \left.\left|P\left(x_{1}+i y\right) e^{x_{1}} e^{\delta+\varepsilon} e^{-\varepsilon / 2}\right|-\mid Q\left(x_{1}+i y+\delta+\varepsilon\right)\right) e^{x_{1}+i y+\delta+\varepsilon} \mid \\
= & \left.a \frac{b}{a} e^{\varepsilon / 2}-\mid Q\left(x_{1}+i y+\delta+\varepsilon\right)\right) e^{x_{1}+i y+\delta+\varepsilon} \mid \\
> & b e^{\varepsilon / 2}-b\left(e^{\varepsilon / 2}-1\right) \\
= & b .
\end{aligned}
$$

Analogously, one obtains $\left|\left(P\left(x_{1}+i y+\delta-\varepsilon\right)+Q\left(x_{1}+i y+\delta-\varepsilon\right)\right) e^{x_{1}+i y+\delta-\varepsilon}\right|<b . \square$

\subsection{Approximation of Julia sets}

\subsubsection{Approximation of functions having Baker do- mains}

Proof of Theorem 9a):

For simplicity we will prove the claim only for the case $P(z)=b z^{d}$ for some $d \in \mathbb{N}_{0}, b \in \mathbb{C}^{*}$, but since we only use arguments for "large" values of $|z|$ it will be clear that our arguments hold for the general case as well. 
First, we fix an $n$ and define some constants (for some definitions we assume $n$ to be sufficiently large):

Let

$c=c_{1}+i c_{2}$,

$r=\operatorname{Re} c / 3>0$,

$0<\varepsilon<\min \left\{r / 3, c_{1} / 8,|c|^{2} / c_{1},|c|^{2} / c_{2},|c|^{2} / 2\right\}$,

$k \in \mathbb{N}$ such that $k<\frac{\varepsilon n}{|c|^{2}}$,

$k_{\max } \in \mathbb{N} \cap\left[\frac{\varepsilon n}{|c|^{2}}-1, \frac{\varepsilon n}{|c|^{2}}\right)$,

$K$ be a compact set contained in the Baker domain $B$ of $f$,

$m \in \mathbb{R}^{+}, w \in \mathbb{R}, l \in \mathbb{N}$ such that $f^{l}(K) \subset D(-m+i w, m / 2)=: D_{0}$, $\left(\frac{3 m}{2}+|w|\right)^{d} e^{-m / 4}<\varepsilon /|b|$ and $m>\frac{40}{3} \frac{r+|c|}{c_{1}}$ (the existence of $m, l, w$ is guaranteed by Lemma 7),

$D_{k}=D(-m+i w-k c, m / 2+k r)$ and

$D=D(-n, n-\sqrt{n})$.

Note that $P_{n}(-n)=-n$ and $P_{n}^{\prime}(-n)=1-\frac{c}{n}$. The value $1-\frac{c}{n}$ is located on the segment connecting 1 and $1-c$. Since the real part of $c$ is positive, $1-\frac{c}{n}$ is contained in the unit disk for sufficiently large $n$. Hence, $-n$ is an attracting fixed point for all but finitely many $n$.

The proof amounts to showing that $D_{0}$ is contained in the Fatou set of $P_{n}$ for all but finitely many $n$. We will do this in three steps:

a) $P_{n}\left(D_{k}\right) \subset D_{k+1}$ for all $0 \leq k \leq k_{\max }$ and all but finitely many $n$

b) $P_{n}(D) \subset D$ for all but finitely many $n$

c) $D_{k_{\max }} \subset D$ for all but finitely many

$n$.

a)

Actually, this claim is not too surprising, since the limit function sends a disk $D_{k}$ into the disk $D_{k+1}$. However, we deal with sets tending to $\infty$ (as $k_{\max }$ depends on $n$ ), so the claim is not obvious. First, using our definitions of $k$ and $\varepsilon$ as above we see that

$n-\frac{m}{4}-\frac{k c_{1}}{2}>n-\frac{m}{4}-\frac{n \varepsilon c_{1}}{2|c|^{2}}>\frac{n}{2} \frac{\left|c_{1}\right|}{|c|^{2}} \frac{|c|^{2}}{\left|c_{1}\right|}-\frac{m}{4}=\frac{n}{2}-\frac{m}{4}$

and

$\frac{k}{n} c_{2}^{2}<\frac{k}{n}\left|c^{2}\right|<\varepsilon<c_{1} / 8$ 
and

$\frac{k}{n}\left|c_{2}\right|<\frac{\left|c_{2}\right|}{2} \frac{n \varepsilon}{|c|^{2}}<\frac{n}{2} \frac{\left|c_{2}\right|}{|c|^{2}} \frac{|c|^{2}}{\left|c_{2}\right|}=\frac{n}{2}$.

We have to show that if $|v|=\frac{m}{2}+k r$, then

$\left|P_{n}(v-m+i w-k c)+m-i w+k c+c\right|<\frac{m}{2}+k r+r$.

The following calculations are valid for all sufficiently large $n$.

$$
\begin{aligned}
& P_{n}(v-m+i w-k c)+m-i w+k c+c \\
= & v \frac{n-c}{n}+c \frac{m-i w+k c}{n}+b(v-m+i w-k c)^{d}\left(\frac{n+v-m+i w-k c}{n}\right)^{n} \\
=: & v \frac{n-c}{n}+c \frac{m-i w+k c}{n}+b \tilde{h}(k) .
\end{aligned}
$$

First, we show that $|\tilde{h}(k)|<\frac{\varepsilon}{|b|}$.

$$
\begin{aligned}
|\tilde{h}(k)| & =|v-m+i w-k c|^{d}\left|\frac{n+v-m+i w-k c}{n}\right|^{n} \\
& \leq(|v|+m+|w|+|k c|)^{d}\left|\frac{n+v-m+i w-k c}{n}\right|^{n} \\
& =\left(\frac{3 m}{2}+|w|+k(r+|c|)\right)^{d}\left|\frac{n+v-m+i w-k c}{n}\right|^{n} \\
& \leq\left(\frac{3 m}{2}+|w|+k(r+|c|)\right)^{d}\left|\frac{\frac{3}{2}|v|+n-m+i w-k c}{n}\right|^{n} \text { for almost all } n \\
& =\left(\frac{3 m}{2}+|w|+k(r+|c|)\right)^{d}\left|\frac{\frac{3}{4} m+\frac{3}{2} k r+n-m+i w-k c}{n}\right|^{n} \\
& =\left(\frac{3 m}{2}+|w|+k(r+|c|)\right)^{d}\left|\frac{n-\frac{m}{4}+i w+k\left(\frac{3}{2} r-c\right)}{n}\right|^{n}=: h(k)
\end{aligned}
$$

$(h(k))^{2}$ can be extended to a differentiable function from $\mathbb{R}^{+}$into itself,

$$
\begin{aligned}
h^{2}(x) & =\left(\frac{3 m}{2}+|w|+x(r+|c|)\right)^{2 d}\left(\left(\frac{n-\frac{m}{4}+x\left(\frac{3}{2} r-c_{1}\right)}{n}\right)^{2}+\left(\frac{w-x c_{2}}{n}\right)^{2}\right)^{n} \\
& =\left(\frac{3 m}{2}+|w|+x(r+|c|)\right)^{2 d}\left(\left(\frac{\left.n-\frac{m}{4}-x c_{1} / 2\right)}{n}\right)^{2}+\left(\frac{w-x c_{2}}{n}\right)^{2}\right)^{n}
\end{aligned}
$$

we obtain (again, for all but finitely many $n$ and $0 \leq x \leq k_{\max }$ )

$$
\begin{aligned}
& \frac{1}{2} \frac{d h^{2}(x)}{d x}\left[\left(\frac{3 m}{2}+|w|+x(r+|c|)\right)^{2 d-1}\left(\left(\frac{n-\frac{m}{4}-x c_{1} / 2}{n}\right)^{2}+\left(\frac{w-x c_{2}}{n}\right)^{2}\right)^{n-1}\right]^{-1} \\
= & d(r+|c|)\left(\left(\frac{n-\frac{m}{4}-x c_{1} / 2}{n}\right)^{2}+\left(\frac{w-x c_{2}}{n}\right)^{2}\right)+ \\
& +\left(\frac{3 m}{2}+|w|+x(r+|c|)\right)\left(\frac{n-\frac{m}{4}-x c_{1} / 2}{n}\left(-\frac{c_{1}}{2}\right)-\frac{w-x c_{2}}{n} c_{2}\right) \\
\leq & d(r+|c|)\left(\left(\frac{n-\frac{m}{4}}{n}\right)^{2}+\left(\frac{w-n / 2}{n}\right)^{2}\right)+\left(\frac{3 m}{2}+|w|+x(r+|c|)\right)\left(\frac{-n / 2+m / 4}{n} \frac{c_{1}}{2}-\frac{w c_{2}}{n}+\frac{1}{8} c_{1}\right) \\
= & d(r+|c|)\left(\left(\frac{n-\frac{m}{4}}{n}\right)^{2}+\left(\frac{w-n / 2}{n}\right)^{2}\right)+\left(\frac{3 m}{2}+|w|+x(r+|c|)\right)\left(-\frac{c_{1}}{4}+\frac{1}{8} c_{1}+\frac{c_{1} m / 8-w c_{2}}{n}\right) \\
\leq & d(r+|c|)\left(1+\frac{1}{4}\right)+\left(\frac{3 m}{2}+|w|+x(r+|c|)\right)\left(-\frac{c_{1}}{8}+\frac{c_{1} m}{8 n}-\frac{w c_{2}}{n}\right) \\
\leq & \frac{5}{4} d(r+|c|)-\frac{3}{2} m \frac{c_{1}}{16} \\
< & 0,
\end{aligned}
$$

since $m>\frac{40}{3} \frac{r+|c|}{c_{1}}$.

Now we know that $h(k) \leq h(0)$, and 
$h(0)=\left(\frac{3}{2} m+|w|\right)^{d}\left|\frac{n-m / 4+i w}{n}\right|^{n} \rightarrow\left(\frac{3}{2} m+|w|\right)^{d}\left|e^{i w-m / 4}\right|<\frac{\varepsilon}{|b|}$. Hence, $|\tilde{h}(k)|<\frac{\varepsilon}{|b|}$ for all $0 \leq k \leq k_{\max }$.

We can proceed with

$$
\begin{aligned}
& \left|P_{n}(v-m+i w-k c)+m-i w+k c+c\right| \\
= & \left|v \frac{n-c}{n}+c \frac{m-i w+k c}{n}+b \tilde{h}(k)\right| \\
\leq & |v|\left|\frac{n-c}{n}\right|+|c| \frac{m+|w|}{n}+\frac{k|c|^{2}}{n}+|b||\tilde{h}(k)| \\
< & |v|+|c| \frac{m+|w|}{n}+\frac{k|c|^{2}}{n}+|b||\tilde{h}(k)| \\
< & \frac{m}{2}+k r+\varepsilon+\varepsilon+\varepsilon \\
< & \frac{m}{2}+k r+r .
\end{aligned}
$$

This proves $P_{n}\left(D_{k}\right) \subset D_{k+1}$ for all but finitely many $n$ and $0 \leq k \leq k_{\max }$.

b)

We have to show that if $|v|=n-\sqrt{n}$, then

$$
\begin{aligned}
\left|P_{n}(v-n)+n\right|<n- & \sqrt{n} . \\
& \left|P_{n}(v-n)+n\right| \\
= & \left|v \frac{n-c}{n}+b(v-n)^{d}\left(\frac{v}{n}\right)^{n}\right| \\
= & |v|\left|1-\frac{c}{n}+b(v-n)^{d}\left(\frac{v}{n}\right)^{n-1} \frac{1}{n}\right| \\
= & |v|\left|1+\frac{1}{n}\left(b(v-n)^{d}\left(\frac{v}{n}\right)^{n-1}-c\right)\right| .
\end{aligned}
$$

Now,

$$
\begin{aligned}
& \left|b(v-n)^{d}\left(\frac{v}{n}\right)^{n-1}\right| \\
\leq & \left|\frac{n}{v}\right||b|\left|(2 n)^{d}\left(\frac{n-\sqrt{n}}{n}\right)^{n}\right| \\
= & \left|\frac{n}{v}\right||b|\left|(2 n)^{d}\left(\left(1-\frac{1}{\sqrt{n}}\right)^{\sqrt{n}}\right)^{\sqrt{n}}\right| \\
\leq & \left|\frac{n}{v}\right||b|\left|(2 n)^{d}\left(\frac{1}{e}+\varepsilon_{0}\right)^{\sqrt{n}}\right|,
\end{aligned}
$$

which is small for sufficiently large $n$. We may deduce that $\operatorname{Re} b(v-n)^{d}\left(\frac{v}{n}^{n-1}-c\right)<-\operatorname{Re} c / 2$, by the same argumentation as in the proof that the fixed point is attracting for all but finitely many $n$ we see that $\left|1+\frac{1}{n}\left(b(v-n)^{d}\left(\frac{v}{n}\right)^{n-1}-c\right)\right|<1$ for all but finitely many $n$. This shows part b).

c)

In this part, we have to show that $d\left(-n,-m+i w-k_{\max } c\right)+m / 2+k r<n-\sqrt{n}$. We drop the index, so let $k=k_{\text {max }}$. We have

$\frac{n c_{1}}{|c|^{2}} \frac{4}{3}>\frac{n c_{1}}{|c|^{2}}=\frac{n}{|c|^{2}} \frac{r}{3}>\frac{n \varepsilon}{|c|^{2}}>k$

so with $c_{0}=k r+m / 2+|w|\left(\right.$ recall that $A n-1 \leq k_{\max } \leq A n$ for some $A \in \mathbb{R}^{+}$) 


$$
\begin{array}{rlrl} 
& |n-m-k c+i w|+m / 2+k r & <n-\sqrt{n} \\
\Leftrightarrow|n-m-k c| & <n-\sqrt{n}-k r-m / 2-|w| \\
\Leftrightarrow|n-m-k c| & <n-\sqrt{n}-c_{0} \\
\Leftrightarrow|n-m-k c|^{2} & <\left(n-\sqrt{n}-c_{0}\right)^{2} \\
\Leftrightarrow\left(n-m-k c_{1}\right)^{2}+k^{2} c_{2}^{2} & <\left(n-c_{0}\right)^{2}-2\left(n-c_{0}\right) \sqrt{n}+n \\
\Leftrightarrow n^{2}-2 n\left(m+k c_{1}\right)+\left(m+k c_{1}\right)^{2}+k^{2} c_{2}^{2} & <n^{2}-2 n c_{0}+c_{0}^{2}-2 n \sqrt{n}+2 c_{0} \sqrt{n}+n \\
\Leftrightarrow-2 n\left(m+k c_{1}\right)+\left(m+k c_{1}\right)^{2}+k^{2} c_{2}^{2} & <-2 n c_{0}+c_{0}^{2}-2 n \sqrt{n}+2 c_{0} \sqrt{n}+n .
\end{array}
$$

We only need to consider the terms in which $n$ occurs squared, these are the terms $k n$ and $k^{2}$, since $k$ depends linearly on $n$ :

$$
\begin{array}{rlrl} 
& -2 k c_{1} n+k^{2}|c|^{2} & <-2 n k r+k^{2} r^{2} \\
\Leftrightarrow 0 & <2 n k\left(c_{1}-r\right)-k^{2}\left(|c|^{2}-r^{2}\right) \\
\Leftrightarrow 0 & <2 n k \frac{2}{3} c_{1}-k^{2}\left(|c|^{2}-r^{2}\right) \\
\Leftrightarrow 0 & <\frac{4}{3} c_{1} n-k\left(|c|^{2}-r^{2}\right) \\
\Leftrightarrow k & <\frac{4}{3} \frac{n c_{1}}{|c|^{2}-r^{2}} \\
\Leftrightarrow k & <\frac{4}{3} \frac{n c_{1}}{|c|^{2}} .
\end{array}
$$

This finishes the proof.

\section{Remark 16}

All calculations also work if we use the generalization of Morosawa's sequence as mentioned in the text.

Before proving part b) of the theorem, we need some estimations:

\section{Lemma 30}

Let $P$ be a polynomial of degree $d \in \mathbb{N}_{0}$ with leading coefficient $a \neq 0$. Let $t, K \in \mathbb{R}^{+}$and $S_{n}=\{z: \operatorname{Re} z<-n,|\operatorname{Im} z|<K\}$.

a)

If $\delta \in\left(-\infty, n-d \ln \left(t^{1 / d} n\right)\right)$, then for all large $n$

b)

$\| P(z)\left|\frac{}{D(-n, n-d \ln (t n)-\delta)}<\right| a \mid(2 n)^{d}$.

If $\delta \in\left(-\infty, n-d \ln \left(t^{1 / d} n\right)\right)$, then for all large $n$

c)

$\left\|\left(1+\frac{z}{n}\right)^{n}\right\|_{D\left(-n, n-d \ln \left(t^{1 / d} n\right)-\delta\right)}<n^{-d} \frac{1}{t} e^{-\delta}$.

If $\delta \in \mathbb{R}^{+}$, then for all large $n$

$\min _{D\left(-n, n-d \ln \left(t^{1 / d} n\right)+\delta\right)^{C} \cap \overline{S_{n}}}\left|P(z)\left(1+\frac{z}{n}\right)^{n}\right| \geq 2^{d} \frac{|a|}{t} e^{\delta / 8}$. 


\section{Proof:}

a)

We have $P(z)=a z^{d}+O\left(z^{d-1}\right)$, so

$$
\begin{aligned}
|P(z)| & =|a||z|^{d}+O\left(\left|z^{d-1}\right|\right) \\
& \leq|a||2 n-d \ln (t n)|^{d}+O\left(n^{d-1}\right) \\
& \leq|a||2 n|^{d}-2|a| d \ln (t n)(2 n)^{d-1}+O\left(n^{d-1}\right) \\
& <|a||2 n|^{d} \text { for large } n .
\end{aligned}
$$

b)

We start with the fact that $\ln \left(1-\frac{1}{x}\right)<-\frac{1}{x}$ for all $x \in(1, \infty)$. Hence,

$$
\begin{array}{rlrl} 
& \ln \left(1-\frac{1}{x}\right) & <-\frac{1}{x} \\
\Leftrightarrow x \ln \left(1-\frac{1}{x}\right) & <-1 \\
\Leftrightarrow\left(1-\frac{1}{x}\right)^{x}=\exp \left(x \ln \left(1-\frac{1}{x}\right)\right) & <e^{-1}
\end{array}
$$

and

$$
\begin{aligned}
& \left\|\left(1+\frac{z}{n}\right)^{n}\right\| \frac{}{D\left(-n, n-d \ln \left(t^{1 / d} n\right)-\delta\right)} \\
= & \left(1+\frac{-d \ln \left(t^{1 / d} n\right)-\delta}{n}\right)^{n} \\
= & \left(\left(1-\frac{d \ln \left(t^{1 / d} n\right)+\delta}{n}\right)^{n} \frac{n}{d \ln \left(t^{1 / d} n\right)+\delta}\right)^{d \ln \left(t^{1 / d} n\right)+\delta} \\
< & (\exp (-1))^{d \ln \left(t^{1 / d} n\right)+\delta} \\
= & \frac{1}{\left(n t^{1 / d}\right)^{d}} e^{-\delta} \\
= & \frac{1}{t n^{d}} e^{-\delta}
\end{aligned}
$$

c)

First, we show that $\left(1-\frac{1}{x}\right)^{x}>e^{-1-1 / x}$ for sufficiently large $x \in \mathbb{R}^{+}$. We have

$$
\begin{aligned}
\quad\left(1-\frac{1}{x}\right)^{x}=\exp \left(x \ln \left(1-\frac{1}{x}\right)\right) & >e^{-1-1 / x} \\
\Leftrightarrow x \ln \left(1-\frac{1}{x}\right) & >-1-\frac{1}{x} \\
\Leftrightarrow \ln \left(1-\frac{1}{x}\right)+\frac{1}{x}+\frac{1}{x^{2}} & >0 .
\end{aligned}
$$

Now, $h(x):=\ln \left(1-\frac{1}{x}\right)+\frac{1}{x}+\frac{1}{x^{2}} \rightarrow 0$ as $x \rightarrow \infty$ and $h^{\prime}(x)=\frac{1}{x^{3}}\left(\frac{x}{x-1}-2\right)<0$ for large $x$, so $\left(1-\frac{1}{x}\right)^{x}>e^{-1-1 / x}$ for sufficiently large $x$.

This yields

$$
\begin{aligned}
& \min _{D\left(-n, n-d \ln \left(t^{1 / d} n\right)+\delta\right)^{C}}\left|\left(1+\frac{z}{n}\right)^{n}\right| \\
= & \left(1+\frac{-d \ln \left(t^{1 / d} n\right)+\delta}{n}\right)^{n} \\
= & \left(\left(1-\frac{d \ln \left(t^{1 / d} n\right)-\delta}{n}\right) \frac{n}{+d \ln \left(t^{1 / d} n\right)-\delta}\right)^{d \ln \left(t^{1 / d} n\right)-\delta} \\
> & \left(\exp \left(-1-\frac{d \ln \left(t^{1 / d} n\right)-\delta}{n}\right)^{d \ln \left(t^{1 / d} n\right)-\delta}\right. \\
= & \frac{1}{t n^{d}} e^{\delta} e^{-\frac{\left(d \ln \left(t^{1 / d} n\right)-\delta\right)^{2}}{n}} \\
> & \frac{1}{t n^{d}} e^{\delta / 2} \text { for large } n .
\end{aligned}
$$


Furthermore, we have for large values of $|z|$ that $|P(z)|>|a||z|^{d} e^{-\delta / 4}$ and for $z \in D\left(-n, n-d \ln \left(t^{1 / d} n\right)-\delta\right)^{C} \cap S_{n}$ that $|z| \geq|\operatorname{Re} z| \geq\left|-2 n+d \ln \left(t^{1 / d} n\right)+\delta\right|=\left|2 n-d \ln \left(t^{1 / d} n\right)+\delta\right|$, so $\min _{D\left(-n, n-d \ln \left(t^{1 / d} n\right)-\delta\right)^{C} \cap \overline{S_{n}}}\left|P(z)\left(1+\frac{z}{n}\right)^{n}\right| \geq \frac{|a|\left|2 n-d \ln \left(t^{1 / d} n\right)+\delta\right|^{d}}{t n^{d}} e^{-\delta / 4} e^{\delta / 2}>\frac{|a|}{t} 2^{d} e^{\delta / 8}$

In the following, let $a$ be the leading coefficient of the polynomial $P$, $c \in[1,2), Q_{n}(z)=z-c+P(z)\left(1+\frac{z}{n}\right)^{n}$ and $d$ the degree of $P$.

Proposition 10 (location of attracting fixed points)

Let $\delta, K \subset \mathbb{R}^{+}$and $S_{n}(K)$ as above. Then there exist $\varepsilon, \sigma>0$ such that for all large $n$ and all fixed points $z_{n}$ of $Q_{n}$ in $S_{n}(K)$ the following conditions are satisfied:

a)

b)

$z_{n} \in A_{n}=\overline{D\left(-n, n-d \ln \left(2 n\left(\frac{|a|}{c}\right)^{1 / d}\right)+\delta\right)} \backslash D\left(-n, n-d \ln \left(2 n\left(\frac{|a|}{c}\right)^{1 / d}\right)-\delta\right)$.

c)

$\left|Q_{n}^{\prime}\left(z_{n}\right)\right|<1-\sigma$.

$D\left(z_{n}, \varepsilon\right)$ is contained in the immediate attracting basin of $z_{n}$.

\section{Proof:}

a)

All fixed points $z_{n}$ of $Q_{n}$ satisfy $\left|P\left(z_{n}\right)\left(1+\frac{z_{n}}{n}\right)^{n}\right|=c$. Hence, the claim follows from the preceding lemma.

b)

We have $Q_{n}\left(z_{n}\right)=z$, so $P\left(z_{n}\right)\left(1+\frac{z_{n}}{n}\right)^{n}=c$ and

$Q_{n}^{\prime}\left(z_{n}\right)=1+\frac{c}{1+z_{n} / n}+P^{\prime}\left(z_{n}\right)\left(1+\frac{z_{n}}{n}\right)^{n}$.

By Lemma 30 and a), $\left|1+\frac{z_{n}}{n}\right| \leq \frac{c}{2^{d}|a| n^{d}}$, and $P^{\prime}$ is a polynomial of degree $d-1$, so we may assume that $\left|P^{\prime}\left(z_{n}\right)<(2 n)^{d-1}\right|$. Hence, $\left|P^{\prime}\left(z_{n}\right)\left(1+\frac{z_{n}}{n}\right)^{n}\right|<\frac{R}{n}$ for some $R>0$.

By a) we can write $z_{n}=-n+r n e^{i \phi}$, where

$r \in\left(-1+\frac{d \ln \left(\frac{2|a| n}{c}\right)}{n}-\frac{\delta}{n},-1+\frac{d \ln \left(\frac{2|a| n}{c}\right)}{n}\right)$ and $|\phi|<\varepsilon_{n}$, where $\varepsilon_{n} \rightarrow 0$ as $n \rightarrow \infty$. Let $\delta=2-c>0$. If $n$ is sufficiently large, we have $\left|\frac{c}{r}\right|<|c|+\frac{\delta}{2}=2-\frac{\delta}{2}$, and we are done with noting that if $|\phi|$ is sufficiently small and $n$ large, then the value $1-\left(2-\frac{\delta}{2}\right) e^{i \phi}+\frac{R v}{n}$ is contained in the disk $D\left(0,1-\frac{\delta}{4}\right)$ for all $v \in \mathbb{D}$. 
c)

We calculate

$Q_{n}^{\prime \prime}(z)=\left(1+\frac{z}{n}\right)^{n}\left(\left(\frac{n-1}{n}\right) P(z)\left(1+\frac{z}{n}\right)^{-2}+2 P^{\prime}(z)\left(1+\frac{z}{n}\right)^{-1}+P^{\prime \prime}(z)\right)$. For $\delta>0$, large $n$ and

$z \in D\left(-n, n-d \ln \left(2 n\left(\frac{|a|}{c}\right)^{1 / d}\right)+\delta\right) \backslash D\left(-n, n-d \ln \left(2 n\left(\frac{|a|}{c}\right)^{1 / d}\right)-\delta\right)$ we have $\left|1+\frac{z}{n}\right|>1 / 2$, so $Q_{n}^{\prime \prime}(z)<\left|1+\frac{z}{n}\right|^{n}\left(4|P(z)|+4\left|P^{\prime}(z)\right|+\left|P^{\prime \prime}(z)\right|\right)$.

From the estimations in Lemma 30 we obtain a $M>0$ such that for a sufficiently small $\varepsilon>0$ the inequation $\left|Q_{n}^{\prime \prime}(z)\right|<M$ holds for all large $n$ and $z \in D\left(z_{n}, \varepsilon\right)$.

By b) and decreasing $\varepsilon$ if necessary we may assume that $\left|Q_{n}^{\prime}(z)\right|<1$ on $D\left(z_{n}, \varepsilon\right)$. This proves part c).

We fill this proposition with life by proving

Lemma 31 (Existence of fixed points in $S_{n}(K)$ )

Let $A_{n}$ be as above, then we can find a $K>0$ such that $A_{n} \cap S_{n}(K)$ contains at least two fixed points of $Q_{n}$ for large $n$.

\section{Proof:}

Let $\tilde{Q}_{n}(z)=Q_{n}(z)-(z-c)=P(z)\left(1+\frac{z}{n}\right)^{n}$ and $\gamma_{n}=\tilde{Q}_{n}^{-1}(\partial(D(0, c))$. Then $\gamma_{n}$ is a union of finitely many closed curves containing all fixed points of $Q_{n}$, and $\gamma_{n} \cap S_{n}(K) \subset A_{n}$ for any fixed $K$ and large $n$.

We have $\tilde{Q}_{n}^{\prime}(z)=Q_{n}^{\prime}(z)-1=\left(\frac{P(z)}{1+z / n}+P^{\prime}(z)\right)\left(1+\frac{z}{n}\right)^{n}$. By using the estimations from Lemma 30 we can find a constant $M>0$ such that $\min _{z \in \gamma_{k} \cap \overline{S_{n}(K)}}\left|\tilde{Q}_{n}^{\prime}(z)\right|=M$, in particular, there are no critical points of $\tilde{Q}_{n}$ in $\gamma_{n} \cap S_{n}(K)$.

Let $K=4 \frac{\pi c}{M}$, then $\gamma_{n} \cap S_{n}(K)$ contains a Jordan curve of length at least $K$ or two Jordan curves of length larger than $K / 2$. Since $\left|\tilde{Q}_{n}^{\prime}<M\right|$ on these curves and these curves are mapped to $\partial D(0, c)$, we obtain at least two points $z_{1} \neq z_{2} \in \gamma_{n} \cap S_{n}(K)$ such that $\tilde{Q}_{n}\left(z_{1}\right)=\tilde{Q}_{n}\left(z_{2}\right)=c$. Hence, $z_{1}$ and $z_{2}$ are fixed points of $Q_{n}$.

Finally, we prove a lemma which enables us to give estimates for preimages:

\section{Lemma 32}

Let $P$ be a polynomial, $\|P\|_{\overline{\mathbb{D}}} \leq 1$ and $f(z)=z+P(z)$. Then $0 \in f(\overline{\mathbb{D}})$.

\section{Proof:}

We only have to deal with the case $0 \notin f(\partial \mathbb{D})$, otherwise the claim is obvious. $\|P\|_{\mathbb{D}} \leq 1$ yields that $f(1)$ is contained in the open right half 
plane, $f(-1)$ is contained in the open left half plane. Let $\gamma_{1}$ be the arc connecting 1 and -1 and containing $i$ and $\gamma_{2}$ the arc connecting 1 and -1 and containing $-i . f\left(\gamma_{1}\right)$ does not intersect the negative imaginary axis, neither does $f\left(\gamma_{2}\right)$ intersect the positive imaginary axis. Hence, $f(\partial \mathbb{D})$ is a closed curve winding around 0 , so $0 \in f(\mathbb{D})$ in this case.

Now we are ready to prove part b) of the theorem.

Proof of Theorem 9b):

Due to the preceding estimations we find two sequences $z_{1}(n), z_{2}(n)$ and $K, \varepsilon>0$ such that for $i=1,2$ and sufficiently large $n$ the following holds:

- $z_{i}(n)$ is an attracting fixed point of $Q_{n}$

- $\left|\operatorname{Im} z_{i}(n)\right|<K$

- $z_{i}(n)+\frac{3 \varepsilon}{4} \in A^{*}\left(z_{i}(n)\right) \cap D\left(-n, n-d \ln \left(2 n\left(\frac{|a|}{c}\right)^{1 / d}\right)-\varepsilon / 2\right)$, where $A^{*}\left(z_{i}(n)\right)$ is the immediate attracting basin of $z_{i}(n)$.

We will show that there exists a compact set $R$ contained in the Baker domain of $f$ such that $R \cap O^{-}\left(z_{i}(n)\right) \neq \emptyset$ for $i=1,2$. This implies that $R$ contains points of the Julia set of $Q_{n}$ for infinitely many $n$, so $\operatorname{dist}\left(J\left(Q_{n}\right), J(f)\right)>\operatorname{dist}(R, \partial B)>0$.

The general idea is to iterate the values $z_{i}(n)+\frac{3 \varepsilon}{4}$ backwards, by showing that $Q_{n}$ is "close " to the translation by $-c$ on some domain in the left half plane we see that there exists a branch of the inverse function of $Q_{n}$ which is "close" to the translation by $c$. Hence, the backward iterates move "almost" horizontally, and by estimating the imaginary parts of the backward iterates we will obtain the existence of our desired set $R$. The technical main problem is to quantify the words "close" and "almost" above.

First, let us define some constants:

Let $\delta$ be the unique value in $\mathbb{R}^{+}$such that $\delta=c-\exp \left(-\frac{\delta}{2}-\frac{\varepsilon}{2}\right)$,

$K$ such that $\operatorname{Im}\left|z_{i}(n)\right|<K$ as above,

$K_{1}=e^{-\varepsilon / 2} \sum_{s=1}^{\infty}\left(e^{-\delta}\right)^{i} \in(0, \infty)$,

$\tilde{K}=K+K_{1}+2$,

$M=\frac{3}{2}|a| e \sum_{i=0}^{\infty}(1+i)^{2} e^{-i}<\infty$,

$\alpha_{0}<-2$ such that the rectangle 
$R=\left\{z: \alpha_{0}-2 c-1<\operatorname{Re} z<\alpha_{0}-1,|\operatorname{Im} z|<\tilde{K}\right\}$ is contained in the Baker domain of $f,\left|\alpha_{0}\right|^{d} e^{\alpha_{0}} M<1$ and $\frac{3}{2}|a|\left(x^{2}+K^{2}\right)^{d / 2} e^{x} e^{\tilde{K}}<\frac{3}{2}$ if $x<\alpha_{0}$,

$C_{0}>\tilde{K}$ such that for any $z$ satisfying $z \in D\left(-n, C_{0}\right), z+c \notin D\left(-n, C_{0}\right)$ and $|\operatorname{Im} z|<\tilde{K}$ we have $\operatorname{Re} z>-n+C_{0}-2 c$ and $n$ large such that

- $\| P(z)\left|\frac{}{D(0,2 n-d \ln (c n))}<\right| a \mid(2 n)^{d}$ and

- $|a|(2 n)^{d}\left(\frac{C_{0}}{n}\right)^{n}<\frac{c}{2 c+2}$ and

- if $\sigma>C_{0}, z \in D(-n, \sigma),|\operatorname{Im} z|<\tilde{K}$, Re $z<-n$, then $D\left(z+c, \exp \left(-\frac{\delta}{2}-\frac{\varepsilon}{2}\right)\right) \subset D\left(-n, \sigma-\frac{\delta}{2}\right)$

(possibly we have to increase $C_{0}$ to obtain the last property).

Let $z_{0} \in\left\{z_{1}(n)+\frac{3}{4} \varepsilon, z_{2}(n)+\frac{3}{4} \varepsilon\right\}$.

\section{Step 1:}

We have $z_{0} \in D\left(-n, n-d \ln \left(2 n\left(\frac{|a|}{c}\right)^{1 / d}\right)-\varepsilon / 2\right)$, and by our hypothesis we see that $D_{1}=D\left(z_{0}+c, \exp \left(-\frac{\delta}{2}-\frac{\varepsilon}{2}\right)\right) \subset D\left(-n, n-d \ln \left(2 n\left(\frac{|a|}{c}\right)^{1 / d}\right)-\varepsilon / 2-\delta / 2\right)$. Using Lemma 30 we see that $\left\|Q_{n}(z)-(z-c)\right\|_{\overline{D_{1}}}<\exp \left(-\frac{\delta}{2}-\frac{\varepsilon}{2}\right)=\operatorname{rad}\left(D_{1}\right)$. Hence, $\partial D_{1}$ is mapped by $Q_{n}$ to a closed curve $\gamma$ such that $z_{0} \in$ int $\gamma$, so there is a $z_{1} \in \overline{D_{1}} \cap Q_{n}^{-1}\left(z_{0}\right)$.

We can do the same to $z_{1}$, recall that

$z_{1} \in D\left(-n, n-d \ln \left(2 n\left(\frac{|a|}{c}\right)^{1 / d}\right)-\varepsilon / 2-\delta / 2\right)$, and setting

$D_{2}=D\left(z_{1}+c, \exp \left(-2 \frac{\delta}{2}-\frac{\varepsilon}{2}\right)\right)$ we have

$\left\|Q_{n}(z)-(z-c)\right\|_{D_{2}}<\exp \left(-2 \frac{\delta}{2}-\frac{\varepsilon}{2}\right)=\operatorname{rad}\left(D_{2}\right)$. After finitely many steps we end up with a $v \in O^{-}\left(z_{0}\right) \cap D\left(-n, C_{0}\right)$, provided that the imaginary parts of our backward iterates do not move too much. But for any $z_{j} \in P^{-j}\left(z_{0}\right)$ constructed this way we have

$$
\begin{aligned}
\left|\operatorname{Im} z_{j}-\operatorname{Im} z_{0}\right| & \leq \sum_{s=1}^{j}\left\|Q_{n}(z)-(z-c)\right\|_{D_{s}} \\
& =\sum_{s=1}^{j} \exp \left(-s \frac{\delta}{2}-\frac{\varepsilon}{2}\right) \\
& <e^{-\frac{\varepsilon}{2}} \sum_{s=1}^{\infty}\left(\exp \left(-\frac{\delta}{2}\right)\right)^{s} \\
& =K_{1} .
\end{aligned}
$$

Hence, we have a $v \in O^{-}\left(z_{0}\right) \cap D\left(-n, C_{0}\right) \cap\left\{z:|\operatorname{Im} z|<K+K_{1}\right\}$.

\section{Step 2:}


We have

$$
\begin{aligned}
\left\|Q_{n}(z)-(z-c)\right\|_{\overline{D\left(-n, C_{0}\right)}} & =\left\|P(z)\left(1+\frac{z}{n}\right)^{n}\right\|_{\overline{D\left(-n, C_{0}\right)}} \\
& \leq|a|(2 n)^{d}\left(\frac{C_{0}}{n}\right)^{n} \\
& <\frac{c}{2 c+1}
\end{aligned}
$$

by our hypothesis. Using the same argument as in Step 1, we can iterate $v$ backwards. For every backward iteration, the real part moves at least by $c-\sigma$. We are interested in the value $v^{\prime} \in O^{-}(v)$ obtained by this procedure such that $v^{\prime} \in D\left(-n, C_{0}\right)$ but $v^{\prime}+c \notin D\left(-n, C_{0}\right)$. The number of iterations $m$ we need satisfies $m(c-\sigma)<2 C_{0}+1$. The distance $\left|\operatorname{Im} v-\operatorname{Im} v^{\prime}\right|$ is smaller that $m\left\|Q_{n}(z)-(z-c)\right\| \frac{}{D\left(-n, C_{0}\right)} \leq \frac{2 C_{0}+1}{c-\sigma} \sigma=\frac{2 C_{0}+1}{c-\frac{c}{2 C_{0}+2}} \sigma=1$. We conclude that there is a $v^{\prime} \in O^{-}\left(z_{0}\right) \cap\left\{z:|\operatorname{Im} z|<K+K_{1}+1\right\} \cap D\left(-n, C_{0}\right)$ such that $v^{\prime}+c \in D\left(-n, C_{0}\right)$. Using our hypothesis we see that $\operatorname{Re} v^{\prime}>-n$.

\section{Step 3:}

We continue our backward iteration, using different estimations.

For each $z$ satisfying $\operatorname{Re} z \in\left(-n, \alpha_{0}\right)$ and $|\operatorname{Im} z|<\tilde{K}$ we

have $|z|^{2} \leq \tilde{K}^{2}+\alpha^{2}$, so $|P(z)|^{2} \leq|a|^{2}\left(\frac{3}{2}\right)^{2}\left(\tilde{K}^{2}+\alpha^{2}\right)^{d}$ and $|z+n|<\tilde{K}+\alpha+n$, so $\left|\left(1+\frac{z}{n}\right)^{n}\right|<\left(1+\frac{\tilde{K}+\alpha}{n}\right)^{n}<e^{\alpha+\tilde{K}}$, and we

obtain $\left|P(z)\left(1+\frac{z}{n}\right)^{n}\right|<\frac{3}{2}|a|\left(\tilde{K}^{2}+\alpha^{2}\right)^{d / 2} e^{\alpha+\tilde{K}}<\frac{c}{2}$. If we construct the preimages of $v^{\prime}$ as before and make shure that the imaginary parts always have modulus smaller than $\tilde{K}$, then we obtain a $v_{0} \in O^{-}\left(z_{0}\right) \cap R$ as desired. To estimate the imaginary parts, we do the following:

Note that $h(x)=\frac{3}{2}|a|\left(\left(x+\frac{3}{2} c\right)^{2}+K^{2}\right)^{d / 2} e^{x+\frac{3}{2} c} e^{\tilde{K}}$ is an increasing function on $\left(-\infty, \alpha_{0}\right) \subset(-\infty,-2)$. We construct the first preimage $v_{1}^{\prime}$ of $v^{\prime}$, by our estimations above we know it can be found in the disk $\overline{D\left(v^{\prime}+c, c / 2\right)}$. Let $x_{0}=\operatorname{Re} v^{\prime} \in\left(-n, \alpha_{0}\right)$, then we can calculate the value

$\left\|Q_{n}(z)-(z-c)\right\|_{\overline{D\left(v^{\prime}+c, c / 2\right)}}$ more precisely to be

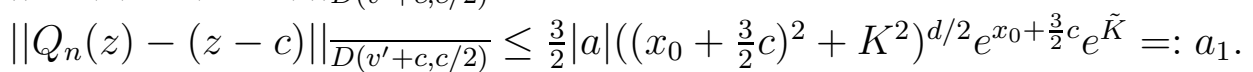

Hence, $v_{1}^{\prime} \in \overline{D\left(v^{\prime}+c, a_{1}\right)}$. The condition $\left|\alpha_{0}^{d} e^{\alpha_{0}}\right| M<1$ guarantees that $a_{1}<1$, so $\left|\operatorname{Im} v_{1}^{\prime}\right|<\tilde{K}$. We repeat this method to obtain a preimage of $v_{1}^{\prime}$, it is contained in the set $\overline{D\left(v_{1}^{\prime}, a_{2}\right)}$, where $a_{2}=\frac{3}{2}|a|\left(\left(x_{0}+\frac{3}{2} c+\frac{3}{2} c\right)^{2}+K^{2}\right)^{d / 2} e^{x_{0}+3 c} e^{\tilde{K}}$. Again, $\left|\alpha_{0}^{d} e^{\alpha_{0}}\right| M<1$ yields $\left|a_{1}+a_{2}\right|<1$, so $\left|\operatorname{Im} v_{2}\right|<\tilde{K}$. We can continue iterating backwards to obtain $v_{i}^{\prime}, i=3, \ldots$ as long as $\operatorname{Re} v_{i}^{\prime}<\alpha_{0}$. Using that $\operatorname{Re} v_{i}^{\prime}-\operatorname{Re} v_{i-1}^{\prime} \in\left(\frac{c}{2}, \frac{3 c}{2}\right)$ as long as $v_{i}^{\prime}$ is defined, we end up with a $v_{0} \in O^{-}\left(z_{0}\right) \cap R$.

Since we can do this with both attracting fixed points, $R$ contains points of two different Fatou components, so it contains points of the Julia set and our proof is complete. 


\subsection{The limiting processes $\operatorname{Re} a c \rightarrow 0$}

\subsubsection{The dynamical plane}

For each $\lambda \in \mathbb{C}^{*}$, the function $g_{\lambda}$ has exactly two singular values, namely the critical value $-\frac{\lambda}{e}$ and the asymptotic value 0 . A result of Eremenko and Lyubich gives that these functions do not have Baker domains or wandering domains, another result in the same paper states that there are at most two non-repelling cycles. The case $c \in\{z: \operatorname{Re} z \geq 0\}$ corresponds to the case $\lambda \in \overline{\mathbb{D}^{*}}$, this is the case we are interested in. Note that 0 is always a fixed point of $g_{\lambda}$, its multiplier is $\lambda$.

In the following, let $\lambda$ and $c$ be such that $\lambda=e^{-c}$.

First, we look at the case $\lambda \in \mathbb{D}^{*}$. 0 is an attracting fixed point of $g_{\lambda}$, the Baker domain of $f_{c}$ is the lift of the immediate attracting basin of 0 . One way to see that this basin contains the critical point -1 (and not only the asymptotic value 0), or, equivalently, that the Baker domain contains all critical points of $f_{c}$, is to use the result of Bergweiler in the introduction, another way is to observe that any attracting (but not super-attracting) basin contains a singular value which is not (pre-)periodic. This implies that if $\lambda \in \mathbb{D}^{*}$, then $F\left(g_{\lambda}\right)$ contains exactly one periodic component, as well as $F\left(f_{c}\right)$ does.

Since all Fatou components of $g_{\lambda}$ are finally mapped into the immediate attracting basin of 0 , all Fatou components of $f_{c}$ are finally mapped into the Baker domain. Hence, $f_{c}$ has no wandering domains.

We turn to the case $\lambda=\exp (2 \pi i p / q)$, where $p / q \in \mathbb{Q}$ and $(p, q)=1$. 0 is a parabolic fixed point, using that there is only one singular value which is contained in the Fatou set we see that the Fatou set consists of one cycle of parabolic domains (and possible preimages). The Fatou set of $f_{0}$ has already been described in [43], [44] or [49], it consists of infinitely many invariant Baker domains contained in horizontal strips of height $2 \pi$, the horizontal lines at 0 and $2 \pi i k, k \in \mathbb{N}$, are contained in the Julia set. For each point in such a Baker domain, the real part tends to $-\infty$ under iteration. Each Baker domain contains exactly one critical value, and all critical values are contained in these Baker domains. Using estimations in [43] one easily verifies that these Baker domains are examples for the second case of König's classification. 
If $c=2 \pi i n$ for some $n \in \mathbb{Z} \backslash\{0\}$, then $J\left(f_{c}\right)=J\left(f_{0}\right)$ by using the lift, and it is easy to see that the Baker domains for $f_{0}$ turn into wandering domains for $f_{c}$. Nothing different happens if more general $\lambda=\exp (2 \pi i p / q), p, q$ as above, but $p \neq 0$; the petals of the parabolic fixed point 0 of $g_{\lambda}$ are lifted to wandering domains of $f_{c}$, this can be observed by choosing a point in a petal and following its forward orbit and lifting this orbit (note that winding once around the origin in the dynamical plane of $g_{\lambda}$ is lifted to a translation by $2 \pi i$ in the dynamical plane of $f_{c}$ ). Here as well, for a $z$ in such a wandering domain, the real part tends to $-\infty$ under iteration, but this time the imaginary part does as well. These wandering domains are Baker wandering domains. Note that if $g_{\lambda}$ has $q$ petals attached to the origin, then for each of these wandering domains $V$ the union $\bigcup_{n=1}^{q} f_{c}^{n}(V)$ contains exactly one critical point if $V$ is a lift of a petal. Conversely, all singular values of $f_{c}$ are contained in wandering domains. Finally, we can use the argumentation from the case $\lambda \in \mathbb{D}{ }^{*}$ to show that $f_{c}$ has no other periodic Fatou components and no other asymptotic path of wandering domains. Hence, the number of asymptotic paths of wandering domains only depends on the number $q$ of petals of $g_{\lambda}$ and the smallest integer $k$ larger than $|p / q|$, it is equal to $k q$.

Next, we will look at the case where 0 is the center of a Siegel disk of $g_{\lambda}$. We will use the following result of Geyer:

Theorem 13 (Geyer, [27])

0 is the center of a Siegel disk of $g_{\lambda}$ if and only if $\lambda$ is contained in the set of Brjuno numbers.

This case has already been described in [3] and [34]. The corresponding functions $f_{c}$ have univalent Baker domains $B_{c}$ containing half planes, they are examples of class 2 in König's classification. Proposition 11 tells us that the Siegel disks are the only periodic Fatou components of $g_{\lambda}$, and as above we see that the Baker domains are the only periodic Fatou components of $f_{c}$ and that $f_{c}$ has no wandering domains.

It only remains to check the case where 0 is an irrationally indifferent non-linearizable fixed point. Theorem 11 tells us that $g_{\lambda}$ has no Siegel disks for such a $\lambda$. Since 0 is a Cremer point, the forward orbit of the critical value has to accumulate at 0 (see the introduction), so it cannot be contained in an attracting or parabolic basin. Hence, the Fatou set is empty, and we also obtain that $J\left(f_{c}\right)=\overline{\mathbb{C}}$. 


\section{Proposition 11}

If $g_{\lambda}$ has an irrationally indifferent fixed point at 0 then there is no (other) Siegel disk.

The idea behind this proposition is that the boundary of a Siegel disk is contained in the closure of the post-singular set, while Cremer points are contained in the derived set of the post-singular set. $g_{\lambda}$ has only two singular values, one of them, namely 0 , is fixed. If the theorem would be false, then the other singular value would accumulate at the Cremer point and at any point of the boundary of the Siegel disk or at any point of the boundary of both Siegel disks, respectively. We will show that such a "part time job" is impossible (even the case that both Siegel disks have the same boundary is not possible).

\section{Proof:}

Shishikura proved in [50] the sharp bound of non-repelling cycles of rational functions, a generalization of his method for entire transcendental is sketched in [17]. We will follow these arguments, the main idea is to perturb the function in question to make an indifferent periodic point attracting while leaving the number of singularities of the inverse function invariant.

Suppose that $g_{\lambda}$ has an irrationally indifferent fixed point at 0 and a cycle of Siegel disks $\left\{S_{1}, \ldots, S_{p}\right\}$ with centers $\left\{z_{1}, \ldots, z_{p}\right\}$ not containing 0 . Let $z_{0}$ be a preimage of $z_{1}$ not contained in the indifferent cycle (such a point exists, since 0 is the only value having only finitely many preimages under $\left.g_{\lambda}\right)$ and let $R$ be the map $R: \overline{\mathbb{C}} \rightarrow \overline{\mathbb{C}} ; z \mapsto \frac{1}{z-z_{0}}$. We can find a polynomial $h$ of degree $m$ such that $h(0)=0, h\left(-\frac{1}{z_{0}}\right)=0, h\left(R\left(z_{i}\right)\right)=0, h^{\prime}\left(-\frac{1}{z_{0}}\right)=0$ and $h^{\prime}\left(R\left(z_{i}\right)\right)=-1(i=1, \ldots, p)$. Let $\rho: \mathbb{R}_{0}^{+} \rightarrow[0,1]$ be a $C^{\infty}$-function such that $\rho(x)=1$ on $[0,1]$ and $\rho(x)=0$ on $[2, \infty)$. Then $H_{\varepsilon}(z)=z+\varepsilon^{m+1} h(z) \rho(\varepsilon|z|)$ is a quasiconformal homeomorphism for small values of $\varepsilon$, in particular, $H_{\varepsilon}$ has no critical points (the calculations can be found in [36]). Furthermore, $H_{\varepsilon} \rightarrow$ id uniformly as $\varepsilon \rightarrow 0$. $H_{\varepsilon}$ is analytic in $D\left(0, \frac{1}{\varepsilon}\right) \cup D\left(0, \frac{2}{\varepsilon}\right)^{C}$.

$R$ maps the disk $D\left(z_{0}, \varepsilon\right)$ into the set $D\left(0, \frac{1}{\varepsilon}\right)^{C}$. Let $D_{\varepsilon}$ be the attracting basin of $g_{\lambda} \circ R^{-1} \circ H_{\varepsilon} \circ R$ containing $z_{1}$, and $r_{\varepsilon}=d\left(z_{1}, \partial D_{\varepsilon}\right)$. It can be shown that for any $\alpha>0$ there exists a $K(\alpha)>0$ such that $r_{\varepsilon}>K(\alpha) \varepsilon^{\alpha}$ for sufficiently small values of $\varepsilon$ (for a way how to prove this see [36]). Hence, $g_{\lambda} \circ R^{-1} \circ H_{\varepsilon} \circ R\left(D\left(z_{0}, \varepsilon\right)\right) \subset D_{\varepsilon}$ for sufficiently small $\varepsilon$.

We define $f_{\varepsilon}=g_{\lambda} \circ R^{-1} \circ H_{\varepsilon} \circ R$. Note that $R^{-1}\left(H_{\varepsilon}^{-1}\left(R\left(\mathbb{R}^{-}\right)\right)\right)$is an asymptotic path of the asymptotic value 0 of $f_{\varepsilon}$. Summing up, $R^{-1} \circ H_{\varepsilon} \circ R$ and $f_{\varepsilon}$ have the following properties: 
- $R^{-1} \circ H_{\varepsilon} \circ R$ is a quasiconformal homeomorphism, conformal in $\mathbb{C} \backslash D\left(z_{0}, \varepsilon\right)$ and $D\left(z_{0}, \frac{\varepsilon}{2}\right)$

- $\left\{z_{1}, \ldots, z_{p}\right\}$ is an attracting cycle of $f_{\varepsilon}, 0$ is an irrationally indifferent fixed point

- $f_{\varepsilon}$ has two singular values, one asymptotic value which is fixed and one critical value

- $f_{\varepsilon}\left(D\left(z_{0}, \varepsilon\right)\right)$ is contained in an attracting basin of $f_{\varepsilon}$.

Note that all iterates of $f_{\varepsilon}$ are $K$-quasi-regular with a fixed $K$, since $f_{\varepsilon}$ maps the disk $D\left(z_{0}, \varepsilon\right)$ into $\overline{\mathbb{C}} \backslash D\left(z_{0}, \varepsilon\right)$, where $f_{\varepsilon}$ is holomorphic, and the values stay there forever. Hence, we may use the generalized qc-lemma mentioned in the introduction and obtain an entire map having the same dynamical properties. But the attracting cycle requires a singular value contained in an attracting basin such that the forward iterates are bounded away from the Julia set. The other singular value is fixed, but under these circumstances it is impossible to have another irrationally indifferent fixed point.

\subsubsection{Radial continuity of Julia sets}

To prove Theorem 11 we will establish the corresponding result for the functions $g_{\lambda}$. We will have to distinguish between the possible types of the fixed point 0 .

Proposition 12 If $g_{\lambda}(z)$ has a fixed Siegel disk centered at 0, then the sets $J\left(g_{t \lambda}\right), t \in(0,1)$, converge to $J\left(g_{\lambda}\right)$ as $t$ approaches 1 .

\section{Proof:}

Yoccoz proved the analogous result for the family $P_{a}(z)=a z+z^{2}$ in [54], his result was generalized for a larger class of rational functions in [36]. We will adapt this method to our case, closely following the exposition in [36]. 
First, we note that the Siegel disk is the only periodic Fatou component of $g_{\lambda}$ by Proposition 11, and 0 is an attracting fixed point of $g_{t \lambda}$ for $t \in(0,1)$. We have a linearizing function $\Psi$ with the following properties:

1. $\Psi: \mathbb{D} \rightarrow S$ is biholomorphic

2. $g_{\lambda}(\Psi(z))=\Psi(\lambda z)$

3. $\Psi(0)=0$.

We will prove that for any $\varepsilon>0$ there exists a $t_{0} \in(0,1)$ such that $1>t>t_{0}$ implies $\Psi(D(0,1-\varepsilon)) \subset A_{t \lambda}^{*}$, where $A_{t \lambda}^{*}$ is the immediate attracting basin of the attracting fixed point 0 of $g_{t \lambda}$.

Fix an $\varepsilon>0$, we write $r^{\prime}=1-\varepsilon, r^{\prime \prime}=1-\frac{\varepsilon}{2}$ and $s=1-t$ for $t \in \mathbb{C}$. By continuity, for any $n \in \mathbb{N}$ we have a $\delta=\delta(n, \varepsilon)>0$ such that

$$
|1-t|<\delta,|z| \leq r^{\prime \prime}, m \in\{0, \ldots, n\} \text {, implies } g_{t \lambda}^{m}(\Psi(z)) \in S \text {. }
$$

Fix an $n>0$. For any $m \in\{0, \ldots, n\}$ the functions

$y_{m}: D(0, \delta) \times D\left(0, r^{\prime \prime}\right) \rightarrow S ;(s, z) \mapsto g_{t \lambda}^{m}(\Psi(z))$

and

$x_{m}: D(0, \delta) \times D\left(0, r^{\prime \prime}\right) \rightarrow \mathbb{D} ;(s, z) \mapsto \Psi^{-1}\left(y_{m}(s, z)\right)$

are well-defined and holomorphic.

Some calculations using 2. and $g_{(1-s) \lambda}(z)=(1-s) g_{\lambda}(z)$ give $x_{m}(0, z)=\lambda^{m} z$,

$$
\begin{gathered}
\frac{\partial x_{n}}{\partial s}(0, z)=\frac{\partial y_{n}}{\partial s}(0, z) \frac{1}{\Psi^{\prime}\left(x_{n}(0, z)\right)} \\
\frac{\partial y_{m}}{\partial s}(0, z)=-y_{m}(0, z)+g_{\lambda}^{\prime}\left(y_{m-1}(0, z)\right) \frac{\partial y_{m-1}}{\partial s}(0, z)
\end{gathered}
$$

and

$$
g_{\lambda}^{\prime}\left(y_{m-1}(0, z)\right)=\lambda \frac{\Psi^{\prime}\left(x_{m}(0, z)\right)}{\Psi^{\prime}\left(x_{m-1}(0, z)\right)},
$$

each for $z \in D\left(0, r^{\prime \prime}\right)$ and $m \in\{1, \ldots, n\}$. 
The function $k: \mathbb{D} \rightarrow \mathbb{C} ; z \mapsto \frac{\Psi(z)}{z \Psi^{\prime}(z)}$ is analytic, and using the recursion in (4.3), that $y_{m}(0, z)=\Psi\left(x_{m}(0, z)\right)$, (4.2) and (4.4) we obtain $\frac{\partial x_{n}}{\partial s}(0, z)=-\lambda^{n} z \sum_{i=1}^{n} k\left(\lambda^{i} z\right)$.

Applying Birkhoff's ergodicity theorem in [37] yields $\lim _{n \rightarrow \infty} \frac{1}{n} \sum_{i=1}^{n} k\left(\lambda^{i} z\right)=\frac{1}{2 \pi} \int_{0}^{2 \pi} k\left(|z| e^{i \phi}\right) d \phi$.

The mean value theorem gives

$\lim _{n \rightarrow \infty} \frac{1}{n} \sum_{i=1}^{n} k\left(\lambda^{i} z\right)=k(0)=1$ uniformly on $D\left(0, r^{\prime}\right)$.

Hence, for any $\sigma>0$ we can find an $N_{0}=N_{0}(\sigma)>0$ such that

$$
\left|\frac{\partial x_{N}}{\partial s}(0, z)+N \lambda^{N} z\right|=\left|\frac{\partial x_{N}}{\partial s}(0, z)+N x_{N}(0, z)\right|<N \sigma
$$

for $z \in \overline{D\left(0, r^{\prime}\right)}$ and $N>N_{0}$.

The analiticity of the $x_{n}(n=0, \ldots, N)$ on $D(0, \delta(N, \varepsilon)) \times D\left(0, r^{\prime \prime}\right)$ implies for each $\sigma>0$ the existence of a $s_{0}>0$ such that

$$
s \leq s_{0},|z| \leq r^{\prime} \Rightarrow\left|x_{N}(s, z)-x_{N}(0, z)-s \frac{\partial x_{N}}{\partial t}(0, z)\right| \leq s \sigma \text { and } s N<1 / 4 .
$$

Using (4.5) and (4.6) we obtain for $|z|=r^{\prime}$ that

$r^{\prime}\left|\frac{\partial x_{N}(s, z)}{x_{N}(0, z)}-1+s N\right| \leq 2 N s \sigma$.

We choose $\sigma=\frac{r^{\prime}}{4}$ and obtain $\frac{x_{N}(s, z)}{x_{N}(0, z)} \in \mathbb{D}$ for $s<s_{0}$. This means that $g_{(1-s) \lambda}(\Psi(D(0, r "))) \subset \Psi(D(0, r "))$ for $s<s_{0}$ and we are done.

\section{Remark 17}

This proof does not use specific properties of $f$, so it can be used to prove that for any entire function $f$ having a fixed Siegel disk $S$ at 0 the immediate attracting basins of 0 of $(1-\varepsilon) f$ converge to $S$ as $\varepsilon$ tends to 0 .

To handle the parabolic case, we will follow a method of McMullen in [40]. All of his arguments work in our case as well, for the convenience of the reader we will give an outline of the proof. Note the following definitions and theorems given by McMullen, we will restrict them to our special case, for more generality see McMullen's paper. 
Definition 4 (multiplicity, dominant convergence)

Let $f_{n}$ be a sequence of entire functions converging uniformly on compact subsets to a function $f$.

a)

If $f(0)=0, f^{\prime}(0)=1, f^{(i)}(0)=0, f^{(r)}(0) \neq 0$ for $1<i<r$, then $r$ is called the multiplicity of the parabolic fixed point 0 , denoted with mult $(f, 0)$. b)

We say that $\left(f_{n}, 0\right)$ converges to $(f, 0)$ dominantly if $f_{n}(0)=0$ for all but finitely many $n$ and if there exists an $M>0$ such that

$$
\left|f_{n}^{(i)}(0)\right| \leq M\left|f_{n}^{\prime}(0)-1\right| \text { for } 1<i<\operatorname{mult}(f, 0) .
$$

c)

Let $f_{n}(0)=0$ for all but finitely many $n$ and $\left(f^{\prime}(0)\right)^{q}=1$ and $q$ minimal with this property. We say that $\left(f_{n}, 0\right)$ converges to $(f, 0)$ dominantly if $\left(f_{n}^{q}, 0\right)$ converges to $\left(f^{q}, 0\right)$ dominantly.

Definition 5 (horocyclically)

Let $\lambda_{n} \rightarrow 1$ in $\mathbb{C}^{*}$, where $\lambda_{n}=\exp \left(L_{n}+i \theta_{n}\right)$ with $\theta_{n} \rightarrow 0$. We say that $\lambda_{n} \rightarrow 1$ horocyclically if $\frac{\theta_{n}^{2}}{L_{n}} \rightarrow 0$.

Theorem 14 (Theorem 7.2 in [40], dominant normal form) Suppose $\left(f_{n}, 0\right) \rightarrow(f, 0)$ dominantly, mult $(f, 0)=r>1$. Then after passing to a subsequence and making a change of coordinates we can assume that

$$
\begin{gathered}
f_{n}(z)=\lambda_{n} z+z^{r}+O\left(z^{r+1}\right) \\
f(z)=z+z^{r}+O\left(z^{r+1}\right) .
\end{gathered}
$$

Theorem 15 (Theorem 8.2 in [40])

Let $f_{n} \rightarrow f$ on a neighborhood of $\infty$ where

$$
\begin{gathered}
f_{n}(z)=\lambda_{n} z+1+O(1 / z) \\
f(z)=z+1+O(1 / z),
\end{gathered}
$$

and $\lambda_{n} \rightarrow 1$ horocyclically. Then for any $\varepsilon>0$, there are $(1+\varepsilon)$-quasiconformal maps $\phi_{n} \rightarrow \phi$ defined near $\infty$ and conjugating $f_{n} \rightarrow f$ to $T_{n} \rightarrow T$, where

$$
\begin{gathered}
T_{n}(z)=\lambda_{n} z+1 \\
T(z)=z+1 .
\end{gathered}
$$


Theorem 16 (Theorem 8.3 in [40])

Let $f_{n} \rightarrow f$ on a neighborhood of $\infty$ where

$$
\begin{gathered}
f_{n}(z)=\lambda_{n} z+z^{1-p}+O\left(1 / z^{p}\right) \\
f(z)=z+z^{1-p}+O\left(1 / z^{p}\right),
\end{gathered}
$$

$p \geq 1$ and $\lambda_{n} \rightarrow 1$ horocyclically. Then for any $\varepsilon>0$, there are $(1+\varepsilon)$-quasiconformal maps $\phi_{n}$, $\phi$ defined near $\infty$ and conjugating $f_{n} \rightarrow f$ to $T_{n} \rightarrow T$, where

$$
\begin{gathered}
T_{n}(z)=\lambda_{n}\left(z^{p}+1\right)^{1 / p} \\
T(z)=\left(z^{p}+1\right)^{1 / p} .
\end{gathered}
$$

After passing to a subsequence we can assume that $\phi_{n} \rightarrow \phi$.

Lemma 33 (McMullen, Lemma 9.3 in [40])

Let $\lambda_{n} \rightarrow 1$ horocyclically, and let $T_{n}(z)=\lambda_{n} z+1$. Then for any $R>0$ there exists an $N$ such that

$$
\left|T_{n}^{k}(x)\right|>R
$$

whenever $|x|<R$ and $n,|k|>N$.

A short calculations yields that we may apply these theorems.

\section{Lemma 34}

If $\lambda_{0}=\exp (2 \pi i p / q), p / q \in \mathbb{Q}$ and $a_{n}$ is a real sequence converging to 1 from below, then $\left(g_{a_{n} \lambda}^{q}, 0\right)$ converges to $\left(g_{\lambda}^{q}, 0\right)$ dominantly.

\section{Proof:}

Let $\lambda_{n}=\lambda_{0} a_{n}$. We have to show that there exists an $M>0$ such that $\left|\left(g_{\lambda_{n}}\right)^{(i)}(0)\right|<M\left|\left(g_{\lambda_{n}}\right)^{\prime}(0)-1\right|=M\left|\lambda_{n}^{q}-1\right|$ for all $1<i \leq q$.

Fix an $i \in\{2, \ldots, q\}$. We have $g_{\lambda_{n}}^{(i)}(0)=\lambda^{i}$, so the map $\lambda \mapsto\left(g_{\lambda_{n}}\right)^{(i)}(0)$ is a polynomial in $\lambda$. $\lambda_{0}$ is a root of this polynomial and a root of order one of the polynomial $\lambda_{n}^{q}-1$. Using its analyticity we can find an $M_{i}$ such that $\left|g_{\lambda_{n}}^{(i)}(0)\right|<M_{i}\left|\lambda_{n}^{q}-1\right|$ for all $\lambda$ sufficiently close to 0 and our claim follows.

\section{Proposition 13}

If $g_{\lambda}$ has a parabolic fixed point at 0 , then $J\left(g_{(1-\varepsilon) \lambda}\right)$ tends to $J\left(g_{\lambda}\right)$ as $\varepsilon$ tends to 0 . 


\section{Proof:}

First, let $\lambda=1$.

Let $\lambda_{n}$ be a sequence approaching 1 from below. We will show that for any $x \in F\left(g_{\lambda}\right)$ there exists a neighborhood $U$ of $x$ such that $U \subset F\left(g_{\lambda_{n}}\right)$ for all large $n$. Note that we may replace $x$ by some iterate of $g_{\lambda}$ at any stage of the proof. Applying the preceding theorems, we see that there is an $R>0$ such that for all $n>>0$ the linearized dynamics $T_{n}$ on the neighborhood $|z|>R$ of $\infty$ is topologically conjugate to the dynamics of $g_{\lambda_{n}}^{p}$ on a neighborhood of 0 .

The conjugacy $\phi_{n}$ from $g_{\lambda_{n}}$ to $T_{n}$ converges to a conjugacy $\phi$ from $g_{\lambda}$ to $T$. Replacing $x$ with $g_{\lambda}^{i}$ for large $i$ we can assume that $x^{\prime}=\phi(x)$ is defined and satisfies $\operatorname{Re} x^{\prime}>R$ (since the real part increases under iteration by $T$ ). By McMullen's Lemma we can choose a neighborhood $V$ of $x^{\prime}$ and $N>0$ such that

$$
\left|T_{n}^{i}(z)\right|>R \text { for all } z \in V, i>N \text { and } n>N .
$$

For $0<i \leq N$, we have $\left|T^{i}\left(x^{\prime}\right)\right|>R$; since $T_{n} \rightarrow T$, by increasing $N$ we can obtain 4.7 for all $i>0$ and $n>N$.

Since $\phi_{n} \rightarrow \phi$ and $\phi(x) \in V$, there is a neighborhood $U$ of $x$ such that $\phi_{n}(U) \subset V$ for all $n>>0$. By 4.7, the iterates $g_{\lambda_{n}}^{i}(z)$ then remain close to 0 for all $z \in U$ and $i>0$. Thus $\left\{g_{\lambda_{n}}\right\}$ is a normal family, so $U \subset F\left(g_{\lambda_{n}}\right)$ as required.

If $\lambda=\exp (2 \pi i p / q)$, where $p / q \in \mathbb{Q} \backslash \mathbb{N}$, the proof is fairly analogous; by McMullen's Theorems we obtain conjugacies $\phi_{n} \rightarrow \phi$ to the system $T_{n} \rightarrow T$, where

$T_{n}(z)=\lambda_{n}\left(z^{p}+1\right)^{1 / p}$ and $T_{n}(z)=\left(z^{p}+1\right)^{1 / p}$.

Composing these maps with the map $z \mapsto z^{p}$ we obtain a new semiconjugacy $\tilde{\phi}_{n} \rightarrow \tilde{\phi}$ to the system we looked at in the case $\lambda=1$; now we can choose $V$ and $U$ as before and obtain $U \subset F\left(g_{\lambda_{n}}\right)$.

\section{Proof of Theorem 11:}

The cases $\frac{c}{2 \pi i} \in \mathbb{Q}$ and $\frac{c}{2 \pi i} \in \mathcal{B}$ follow from the Propositions 12 and 13; if $\frac{c}{2 \pi i} \notin \mathbb{Q} \cup \mathcal{B}$, then $J\left(f_{c}\right)=\overline{\mathbb{C}}$, and we are finished by noting the theorem in section 3.3.2.

\subsubsection{Approximation of Baker domains and wandering domains}

\section{Proof of Theorem 12:}

The set of irrational values not contained in the Brjuno set is dense in $\mathbb{R}$, so for any $t=\frac{c}{2 \pi i} \in \mathbb{R}$ we can find a sequence $t_{n} \rightarrow t$, where $t_{n} \in \mathbb{R} \backslash(\mathbb{Q} \cup \mathcal{B})$. 
Setting $c_{n}=2 \pi i t_{n}$ we obtain $J\left(f_{c_{n}}\right)=\overline{\mathbb{C}}$, this gives the first claim.

To see c), we use the same sequence $c_{n}$. Recall that for any sequence $P_{n, m}$ converging to $f_{c_{n}}$ we have $J\left(P_{n, m}\right) \rightarrow J\left(f_{c_{n}}\right)=\overline{\mathbb{C}}$. Now we choose a sequence $a_{n}$ tending to $\infty$ sufficiently fast and obtain $J\left(P_{n, a_{n}}\right) \rightarrow \overline{\mathbb{C}} \neq J\left(f_{c_{n}}\right)$.

The proof of $\mathrm{b})$ is very similar; we know that $J\left(f_{c+i \varepsilon}\right) \rightarrow J\left(f_{c}\right)$ and $J\left(P_{c+i \varepsilon, n}\right) \rightarrow J\left(f_{c+i \varepsilon}\right)$, where $P_{c+i \varepsilon, n}=\left(1-\frac{c+i \varepsilon}{n}\right)-c-i \varepsilon+\left(1+\frac{z}{n}\right)^{n}$, so for any sequence $a_{n}$ tending to $\infty$ sufficiently fast we have $J\left(P_{c+i \frac{1}{n}, a_{n}}\right) \rightarrow J\left(f_{c}\right)$.

\subsubsection{Stability}

\section{Proof of Lemma 12:}

In view of our previous results we only have to prove that $f_{c}$ has no Baker domains if Re $c<0$. Again, we first look at the function $g_{\lambda}(z)=\lambda z e^{z}, \lambda=e^{-c}$. 0 is a repelling fixed point of $g_{\lambda}$, so every point $z$ contained in the Fatou set tends to a cycle of attracting or parabolic periodic points not containing 0 or is contained in a cycle of Siegel disks not containing 0. If we consider $f_{c}$ we see that the lift of a point contained in a cycle of attracting or parabolic domains of $g_{\lambda}$ either tends to an attracting or parabolic cycle of $f_{c}$ or tends to $\infty$, but in the latter case it cannot be contained in a periodic Fatou component. Analogously, the lift of a point contained in a Siegel disk of $g_{\lambda}$ may tend to $\infty$, but in this case it enters each Fatou component only once and then enters the next one, so the Fatou set of $f_{c}$ may contain attracting or parabolic cycles, cycles of Siegel disks or wandering domains, but no Baker domains. 


\section{Bibliography}

[1] L. V. Ahlfors. Lectures on quasi-conformal mappings. Van Nostrand, 1966.

[2] I. N. Baker and P. Dominguez. Boundaries of unbounded Fatou components of entire functions. Ann. Acad. Sci. Fenn. Math., 24(2):437-464, 1999.

[3] K. Baranski and N. Fagella. Univalent Baker domains. Nonlinearity, 14:411-429, 2001.

[4] D. Bargmann. Iteration of inner functions and boundaries of components of the Fatou set. Preprint, 1999.

[5] A. F. Beardon. Iteration of rational functions. Springer, New York, Berlin, Heidelberg, 1991.

[6] W. Bergweiler. Iteration of meromorphic functions. Bulletin of the American Mathematical Society, 29:151-188, 1993.

[7] W. Bergweiler. Invariant domains and singularities. Math. Proc. Camb. Phil. Soc., 117:525-532, 1995.

[8] W. Bergweiler. On the Julia sets of analytic self-maps on the punctured plane. Analysis, 15:251-256, 1995.

[9] W. Bergweiler, M. Haruta, H. Kriete, H. G. Meier, and N. Terglane. On the limit functions of iterates in wandering domains. Ann. Acad. Sci. Fenn. Sér. A I Math., 18:151-188, 1993.

[10] W. Bergweiler and Y. F. Wang. Singularities in Baker domains. Computational Methods and Function Theory, 1:41-49, 2001.

[11] M. Büger. On the limit set of functions in wandering domains. Complex Variables Theory Appl., 30, 1996. 
[12] L. Carleson and W. Gamelin. Complex Dynamics. Springer, New York, Berlin, Heidelberg, 1993.

[13] R.L. Devaney and F. Tangerman. Dynamics of entire functiuons near the essential singularity. Ergod. Th. Dynam. Sys., 6:489-503, 1986.

[14] P. Dominguez and G. Sienra. A study of the dynamics of $\lambda \sin z$. Internat. J. Bifur. Chaos Engrg., 12(12):2869-2883, 2002.

[15] A. Douady. Does a Julia Set Depend Continuously on the Polynomial? Proc. Sympos. Appl. Math., 49:91-138, 1994.

[16] A. Douady and J. H. Hubbard. On the dynamics of polynomial-like mappings. Ann. Sci. Ec. Norm. Sup., 18(4):287-343, 1985.

[17] A. E. Eremenko and M. Ju. Lyubich. The dynamics of analytic transforms. Leningrad Math. J., 1:563-634, 1990.

[18] A. E. Eremenko and M. Ju. Lyubich. Examples of entire functions with pathological dynamics. J. London Math. Soc., 1:563-634, 1990.

[19] A. E. Eremenko and M. Ju. Lyubich. Dynamical Properties of some classes of entire functions. Ann. Inst. Fourier, 42:889-1019, 1992.

[20] N. Fagella. Limiting dynamics of the complex standard family. Int. J. of Bif. and Chaos, 3:673-700, 1995.

[21] N. Fagella. Dynamics of the complex standard family. J. of Math. Anal. and Appl., 229:1-31, 1999.

[22] P. Fatou. Sur les équations fonctionelles. Bull. Soc. Math. France, 47:161-271, 1919.

[23] P. Fatou. Sur les équations fonctionelles. Bull. Soc. Math. France, 48:3394, 1920.

[24] P. Fatou. Sur les équations fonctionelles. Bull. Soc. Math. France, 48:208-314, 1920.

[25] P. Fatou. Sur l'itération des fonctions transcendantes entières. Acta Math., 47:337-360, 1926.

[26] L. Geyer. Quasikonforme Deformationen in der Iterationstheorie. Diplomarbeit, TU Berlin, 1994. 
[27] L. Geyer. Siegel discs, Herman rings and the Arnold family. Trans. Math. Soc., 353:3661-3683, 2001.

[28] M. Herman. Are there critical points on the boundary of singular domains? Comm. Math. Phys., 99:593-612, 1985.

[29] A. Hinkkanen. Uniformly quasiregular semigroups. Ann. Acad. Sci. Fenn. Ser. A I, 21:205-222, 1996.

[30] A. Hinkkanen, B. Krauskopf, and H. Kriete. Growing a Baker domain from attracting islands I: The limiting dynamics. To appear, 2004.

[31] X. H. Hua and C. C. Yang. Dynamics of transcendental functions. Gordon and Breach Science Publishers, 1998.

[32] G. Julia. Sur des problèmes concernant l'itération des fonctions rationelles. C. R. Acad. Sci. Paris Sér. I Math., 166:153-156, 1918.

[33] G. Julia. Sur l'itération des fonctions rationelles. J. Math. Pures Appl., $4(7): 47-245,1918$.

[34] H. König. Konforme Konjugation in Baker-gebieten. Dissertation, Universität Hannover, 1996.

[35] H. König. Conformal conjugacies in Baker domains. J. London Math. Soc. (2), 59(1):153-170, 1999.

[36] H. Kriete. Über die Stabilität von Julia-Mengen. Dissertation, Universität Bremen, 1989.

[37] A. Lasota. Probabilistic Properties of Deterministic Systems. Cambridge, 1985.

[38] P. Lavaurs. Systemés dynamique holomorphe, Explosion de points périodique paraboliques. Thése de doctorat, Université Paris-Sud in Orsay, 1989.

[39] O. Lehto and K. I. Virtanen. Quasikonforme Abbildungen. Springer, New York, Berlin, Heidelberg, 1965.

[40] C. T. McMullen. Hausdorff Dimension and Conformal Dynamics II: Geometrically Finite Rational Maps. Comment. Math. Helv., 75:535593, 2000. 
[41] S. Morosawa. The Carathéodory convergence of Fatou components of Polynomials to Baker domains or wandering domains II. Proceedings of the 10th International Conference on Finite Dimensional Complex Analysis and Applications.

[42] S. Morosawa. The Carathéodory convergence of Fatou components of Polynomials to Baker domains or wandering domains. Proc. 2nd ISAAC Congress(eds. H. Begehr, R. P. Gilbert and J. Kajiwara), Kluwer Academic Publishers, 1:347-356, 2000.

[43] S. Morosawa and M. Taniguchi. Non-trivial deformation of an entire function $a b z+e^{b z}+c$. Taiwanese J. Math., 41:641-646, 2001.

[44] S. Morosawa, Nishimura Y., M. Taniguchi, and T. Ueda. Holomorphic Dynamics. Cambridge Studies in Advanced Mathematics, Cambridge University Press, 2000.

[45] R. Oudkerk. The parabolic implosion for $f_{0}(z)=z+z^{v+1}+O\left(z^{v+2}\right)$, 1999.

[46] R. Oudkerk. The parabolic implosion: Lavaurs Maps and Strong Convergence for Rational Maps. Contemp. Math., 303:79-105, 2002.

[47] R. Perez-Marco. Sur une question de Dulac et Fatou. C.R. Acad. Sci. Paris Sér. I Math., 321(8):1045-1048, 1995.

[48] P. J. Rippon and G. M. Stallard. Families of Baker domains I. Nonlinearity, 12(4):1005-1012, 1999.

[49] P.J. Rippon and G. M. Stallard. Families of Baker domains II. Conform. Geom. Dyn., 3:67-78, 1999.

[50] M. Shishikura. On the quasi-conformal surgery of rational functions. Ann. Sci. Ecole. Norm. Sup., 20:1-29, 1987.

[51] M. Shishikura. Bifurcation of parabolic fixed points. London Math. Soc. Lecure Note Ser. 274, pages 325-363, 2000.

[52] G. M. Stallard. Entire functions with Julia sets of measure zero. Math. Proc. Phil. Camb. Soc., 108:551-557, 1990.

[53] D. Sullivan. Conformal dynamical systems. Geometric Dynamics, Proc. Int. Sympos., Rio de Janeiro/Brasil 1981, Lect. Notes Math., 1007:725$752,1983$. 
[54] J. C. Yoccoz. Linéarisation des germes de difféomorphimes holomorphes de (C,0). C.R. Acad. Sci. Paris, 306:55-58, 1988.

[55] J.-H. Zheng. Singularities and limit functions in iteration of meromorphic functions. J. London Math. Soc., 67(2):195-207, 2003. 


\section{Lebenslauf}

Ich wurde am 26.10.1976 in Bremen geboren. Nach dem Besuch des Ökumenischen Gymnasiums zu Bremen legte ich 1996 mein Abitur mit der Note 1.4 ab. Anschließend leistete ich meinen Wehrdienst bei der Sportfördergruppe in Stuttgart ab, bevor ich zum Winteremester 1997/98 das Mathematikstudium in Göttingen aufnahm. Im Juni 2002 bestand ich das Vordiplom, im Juni 2002 legte ich die Diplomprüfungen ab (Note "sehr gut").

Im September 2002 begann ich dann mit der Promotion, während der ich Auslandsaufenthalte in Barcelona/Spanien (September 2002 - August 2003), Paris/Frankreich (September - November 2003), Puebla/Mexiko (Dezember 2003) und Rio de Janeiro/Brasilien (Januar-März 2004) absolvierte. Während dieser Zeit bekam ich ein Marie-Curie-Stipendium der Europäischen Union und Stipendien des DAAD. 